\title{
Electric field stimulation of precision-cut lung slices
}

Von der Fakultät für Mathematik, Informatik und Naturwissenschaften der RWTH Aachen University zur Erlangung des akademischen Grades eines Doktors der Naturwissenschaften genehmigte Dissertation

\author{
vorgelegt von \\ Marco Schlepütz, M.Sc. \\ aus Simmerath \\ Berichter: Priv.-Doz. Dr. Christian Martin \\ Univ.-Prof. Dr. Werner Baumgartner
}

Tag der mündlichen Prüfung: 06. Oktober 2011

Diese Dissertation ist auf den Internetseiten der Hochschulbibliothek online verfügbar. 



\section{Acknowledgement}

The current thesis was made in the Institute of Pharmacology and Toxicology at the University Hospital Aachen, RWTH Aachen University and it is a pleasure for me to thank all the people who made this dissertation possible.

First, I owe my deepest gratitude to Priv.-Doz. Dr. Christian Martin for being my supervisor and mentor. I would like to thank him for his guidance, support, promotion and trust during the whole time. He always had an open door facilitating my work tremendously, and our discussions were always inspiring.

I would also like to thank my second examiner Univ.-Prof. Dr. Werner Baumgartner for his readiness to survey my doctoral studies and his constant interest in my work.

This thesis would not have been possible without the head of the institute Univ.-Prof. Dr. Stefan Uhlig, who provided the requirements to conduct this study. He supported my work strongly with his productive ideas and he promoted my career by giving me the opportunity to attend international conferences as well as to realize a stay in Montreal.

I am grateful to all collaborative partners, who facilitated my work on the species comparison including Sophie Seehase, Dr. Katherina Sewald and Prof. Dr. Armin Braun at the Fraunhofer ITEM in Hannover, Dr. Christina Schlumbohm from the DPZ in Göttingen, Verena Lambermont and Prof. Dr. Boris W. Kramer at the Maastricht University Medical Center, as well as Dr.Jan Spillner, Dr. Alberto Perez-Bouza, Dr. Till Braunschweig, Univ.-Prof. Dr. Rüdiger Autschbach and Univ.-Prof. Dr. Knüchel-Clarke at the University Hospital Aachen.

Kindly, I thank Prof. Dr. James G. Martin, who invited and welcomed me at the Meakins-Christie Laboratories (McGill University, Montreal) to learn the culturing of airway smooth cells, and Dr. Paul-André Risse, who took care of me during the stay.

I am thankful to the German academic exchange service (DAAD) for financial support to attend the ATS International conference 2010 in New Orleans.

Further, I am indebted to many of my colleagues, who were always willing to help and assist me. In this regard I am highly thankful to current and former members of the pulmonary pharmacology group, by name Marc Bernau, Christoph Burghaus, Hanna Czajskowska, Constanze Dassow, Hiltrud Königs, Anke Kowallik, Dennis Lex, Ulrike Maitas, Oliver Pack, Marion Peters, Annette Rieg, Nadine Ruske, Xhina Schneider, Stephanie Siegl, Ulrike Uhlig, Eva Verjans and Yang Yang. Special thanks are due to Kathleen Reiss and UIf Soppa for careful proofreading the manuscript.

To all my friends, not mentioned by name but knowing that they are meant, I am deeply thankful for having an open ear and having motivated me during this study. Especially, I want to thank my girlfriend Sandra Dohmen for her enduring encouragement.

Finally, I profoundly feel obliged to my parents, Inge and Hermann-Josef, as well as my brother Tino, who reliably support me wherever possible. 


\section{Publications}

\section{Original articles:}

Schlepütz M, Uhlig S and Martin C. Electric field stimulation of precision-cut lung slices, J Appl Physiol, 110: 545-554, 2011.

Seehase S, Schlepütz M, Switalla S, Mätz-Rensing K, Kaup FJ, Zöller M, Schlumbohm C, Fuchs E, Lauenstein HD, Winkler C, Kuehl AR, Uhlig S, Braun A, Sewald K and Martin C. Bronchoconstriction in non-numan primates: a species comparison, J Appl Physiol, in press, 2011.

Schlepütz M, Rieg AD, Seehase S, Spillner J, Perez-Bouza A, Braunschweig T, Schroeder T, Bernau M, Lambermont V, Schlumbohm C, Sewald K, Autschbach R, Braun A, Kramer BW, Uhlig S and Martin C. Electric field stimulation of precision-cut lung slices (PCLS) from different mammals - Comparison to responses of human PCLS, Am J Physiol Regul Integr Comp Physiol, submitted

\section{$\underline{\text { Abstracts }}$}

Schlepütz M, Uhlig S and Martin C. Electric field stimulation of precision-cut lung slices, Am J Respir Crit Care Med 179: A2071, 2009.

Seehase S, Schlepütz M, Schlumbohm C, Fuchs E, Kaup FJ, Krug N, Braun A, Martin C and Sewald K. Charakterisierung von Präzisionslungenschnitten aus Marmoset:Vergleich mit humanem Lungengewebe 51. Kongress der deutschen Gesellschaft für Pneumologie und Beatmungsmedizin e.V., Hannover, 2010.

Schlepütz M, Uhlig S and Martin C. Functional analysis of distal lung innervation in precision-cut lung slices Naunyn-Schmiedeberg's Arch Pharmacol 381 (Suppl 1): A255, 2010.

Schlepütz M, Seehase S, Schlumbohm C, Sewald K, Braun A, Kramer BW, Uhlig S and Martin C. Electric field stimulation of precision-cut lung slices suggests differences in distal lung innervation Am J Respir Crit Care Med 181: A5030, 2010.

Schlepütz M, Dassow C, Kramer BW, König P, Uhlig S and Martin C. Comparison of airway responses in sheep of different age in precision-cut lung slices (PCLS), Am J Respir Crit Care Med 181: A5031, 2010. 
Seehase S, Schlepütz M, Schlumbohm C, Fuchs E, Kaup FJ, Krug N, Braun A, Martin C and Sewald K. Characterization of marmoset precision cut lung slices (PCLS): Comparison with human tissue, Am J Respir Crit Care Med 181: A5032, 2010.

Schlepütz M, Bernau M, Uhlig S and Martin C. Electric field stimulation of precision-cut lung slices as a pharmacological tool to study neurally evoked bronchoconstriction NaunynSchmiedeberg's Arch Pharmacol 383 (Suppl 1): P235, 2011.

Seehase S, Schlepütz M, Switalla S, Fuchs E, Kaup FJ, Ressmeyer AR, Uhlig S., Lauenstein HD, Braun A, Martin C and Sewald K. Mechanisms of bronchoconstriction in precision-cut lung slices of non-human primates are comparable to human, Naunyn-Schmiedeberg's Arch Pharmacol 383 (Suppl 1): P257, 2011.

Seehase S, Schlepütz M, Switalla S, Mätz-Rensing K, Schlumbohm C, Habermann G, Fuchs E, Kaup FJ, Ressmeyer AR, Uhlig S, Lauenstein HD, Braun A, Martin C and Sewald K. Humanrelevant bronchoconstrictors are effective in precision-cut lung slices of non-human primates, $\mathrm{Am}$ J Respir Crit Care Med 183: A2584, 2011.

Martin C, Schlepütz $\mathbf{M}$ and Uhlig S. Cross-species comparison on pharmacological and toxicological activities in precision cut lung slices, SOT 50 th annual meeting Washington D.C. 2011

Schlepütz M, Rieg AD, Bernau M, Spillner J, Perez-Bouza A, Uhlig S and Martin C. Comparison of distal lung innervation in human and guinea pig precision-cut lung slices (PCLS), ERS Amsterdam 2011 annual congress Abstract No. 3309, 2011. 


\section{Table of contents}

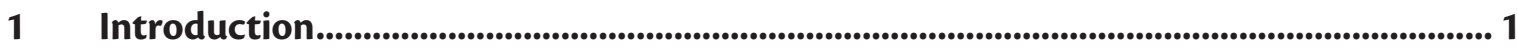

1.1 Importance and principal structure of the lung...............................................................................

1.2 Autonomic innervation of the lung...................................................................................................... 3

1.3 Mechanisms of neurally induced ASM contraction .........................................................................

1.4 Mechanisms of neurally induced ASM relaxation.....................................................................................6

1.5 Electric field stimulation (EFS) of lung tissue preparations in organ baths .............................7

1.6 Precision-cut lung slices (PCLS) ...............................................................................................................

$1.7 \quad$ Asthma and COPD ...................................................................................................................... 10

1.8 Animal models of asthma .............................................................................................................................12

1.9 Neurogenic inflammation and neuronal plasticity ...............................................................................14

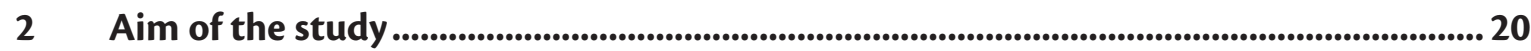

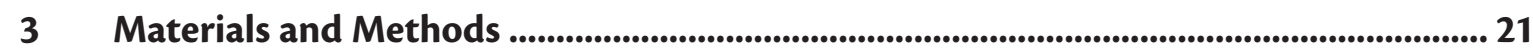

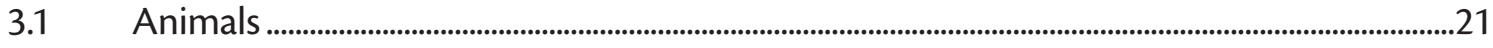

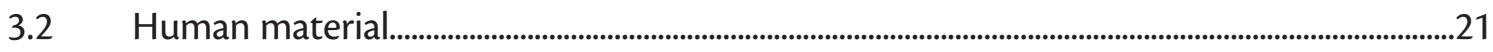

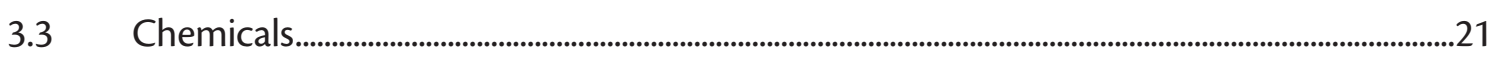

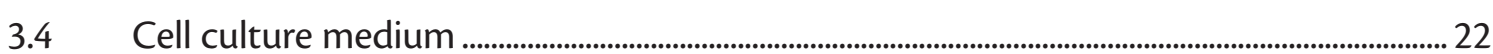

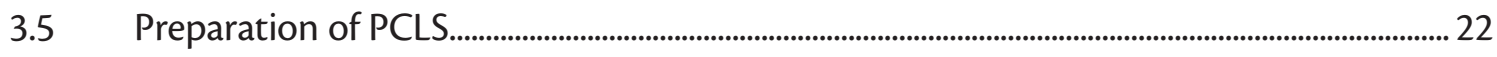

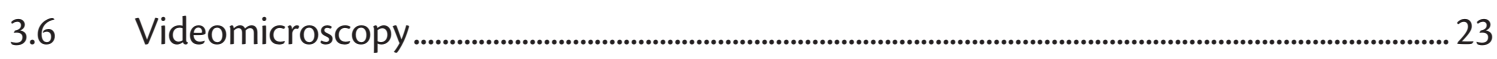

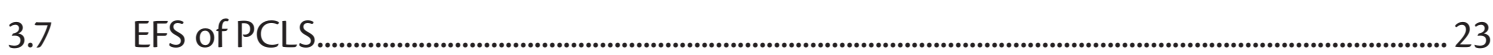

3.8 Realization of response curves for the basic EFS conditions ........................................................24

3.9 Examination of large and small airways in EFS of PCLS ...............................................................24

3.10 Pharmacological interventions .............................................................................................................2

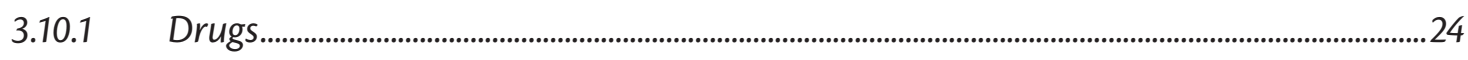

3.10.2 Application of magnesium ......................................................................................................24

3.10.3 Characterization of EFS-induced neural airway responses ..................................................2

3.10.4 Concentration dependent antagonism of anticholinergics ....................................................26

3.10.5 Effect of the TP receptor antagonist SQ29548 ……...............................................................26

3.11 Exogenous activation of airway responses in PCLS ................................................................... 26

3.11.1 Application of methacholine..............................................................................................................26

3.11.2 Application of capsaicin ..................................................................................................................2

3.11.3 Application of U46619..........................................................................................................2

3.12 Active sensitization of rats................................................................................................................................27

3.12.1 Sensitization protocol....................................................................................................................2

3.12.2 EFS of PCLS from sensitized animals ...........................................................................................28

3.12.3 Allergen provocation of PCLS from sensitized animals ........................................................28 
3.12.4 Quantification IgE......................................................................................................................28

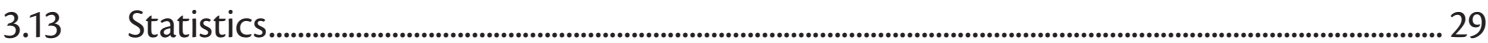

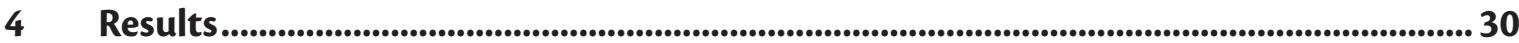

4.1 EFS of PCLS from rats - Design and Development of EFS setup ............................................31

4.1.1 Setup and basic conditions ...................................................................................................................

4.1.2 Cholinergic interventions.................................................................................................................

4.1.3 Effect of magnesium to prove neural activation...........................................................................

4.1.4 Response of different airway sizes....................................................................................................37

4.1.5 TP receptor involvement .........................................................................................................

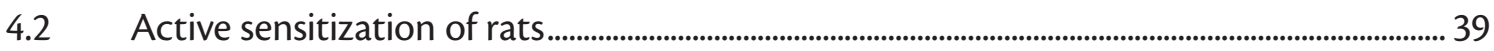

4.2.1 Macroscopic observations ...........................................................................................................................40

4.2.2 Response of PCLS to allergen provocation ................................................................................. 41

4.2.3 Response of PCLS to methacholine ...............................................................................................

4.2.4 IgE levels in sensitization ................................................................................................................. 42

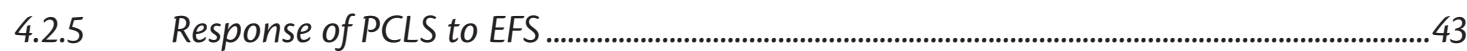

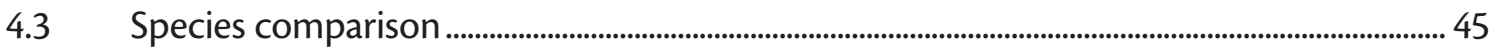

4.3.1 Responses and sensitivity to EFS.................................................................................................45

4.3.2 Evidence for neural activation ......................................................................................................4

4.3.3 Cholinergic antagonism ..................................................................................................................... 48

4.3.4 Activation by capsaicin .......................................................................................................................49

4.3.5 Role of TRP-channels in neurally evoked bronchoconstriction.............................................49

4.3.6 Effect of propranolol and L-NAME in EFS of PCLS from marmoset ...................................50

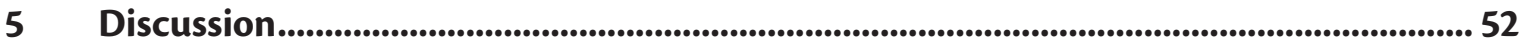

5.1 Design and development of EFS-setup on the basis of rat PCLS ............................................52

5.1.1 General EFS-setup in PCLS..........................................................................................................................5

5.1.2 Relevance of the basic EFS conditions ...............................................................................................53

5.1.3 Cholinergic neuronal response in PCLS......................................................................................5

5.1.4 Reactivity of different airway generations ................................................................................. 55

5.1.5 Role of the TP receptor in neuronal activation ............................................................................55

5.1.6 Comparison to other in vitro models...............................................................................................56

5.2 Active sensitization of rats....................................................................................................................................57

5.2.1 Sensitization scheme ...........................................................................................................................

5.2.2 Airway hyperresponsiveness ...........................................................................................................5

5.2.3 EFS of PCLS from actively sensitized animals ........................................................................60

5.3 Comparison of different species in EFS of PCLS ..........................................................................61

5.3.1 General considerations on EFS of PCLS .....................................................................................61

5.3.2 Pharmacological characterization of distal airway innervation .........................................63 
5.3.3 Phylogenetic considerations........................................................................................................6

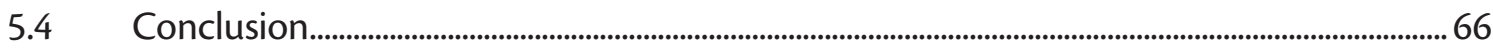

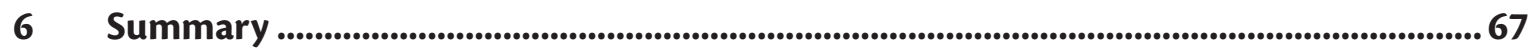

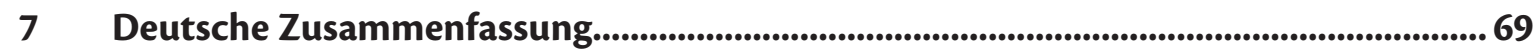

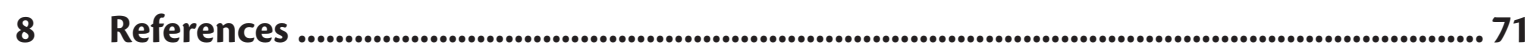




\section{List of abbreviations}

A

AHR

ASM

ATP

$\mathrm{B}$

BDNF

BSA

CAMP

$\mathrm{CBF}$

CGMP

CGRP

COPD

CPI

DAG

4-DAMP

EAR

$\mathrm{EC}_{50}$

$\mathrm{EF}_{50}$

EFS

ELISA

eNANC

$\mathrm{F}$

G

GDNF

GFL

GFR

$G$ protein

GPCR

HDM

HMGB1

IAA

$\mathrm{IC}_{50}$

i.p.

i.tr.

Ig

IL

iNANC
Current amplitude

Airway hyperresponsiveness

Airway smooth muscle

Adenosin triphosphate

Pulse duration

Brain-derived neurotrophic factor

Bovine serum albumin

Cyclic adenosine monophosphate

Ciliary beat frequency

Cyclic guanosine monophosphate

Calcitonin gene related peptide

Chronic obstructive pulmonary disease

PKC activated phosphatase inhibitor

Diacylglycerol

4-diphenylacetoxy- $\mathrm{N}$-(2-chloroethyl)piperidine hydrochloride

Early allergic response

Half-maximal effective concentration

Half-maximal effective frequency

Electric field stimulation

Enzyme linked immunosorbent assay

Excitatory NANC

Frequency

Basic rhythm

Glial-derived neurotrophic factor

GDNF family ligand

GDNF family receptors

Heterotrimeric guanine nucleotide-binding protein

$G$ protein coupled receptor

House dust mite

High-mobility group box 1

Initial airway area

Inhibitory concentration of half-maximal effect

Intraperitoneal

Intratracheal

Immunoglobulin

Interleukin

Inhibitory NANC 


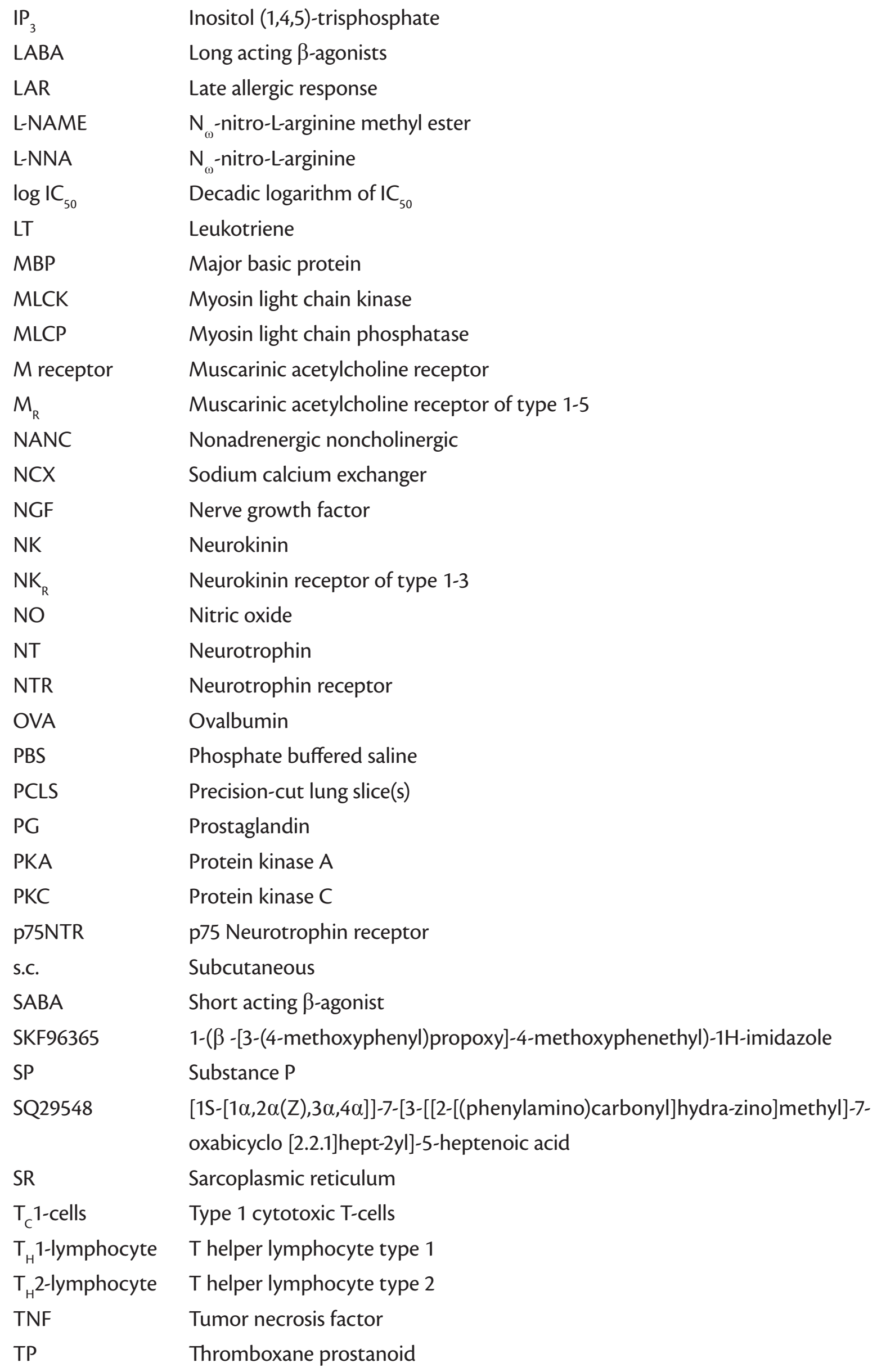


TR

Trk

TRP

TW

TXA

U46619

VIP
Train rhythm

Tropomyosin-related kinase

Transient receptor potential

Train width

Thromboxane

9,11-dideoxy- $9 \alpha, 11 \alpha$-methanoepoxy prostaglandin $F_{2 \alpha}$ Vasoactive intestinal polypeptide 



\section{Introduction}

\subsection{Importance and principal structure of the lung}

The function of the mammalian lung is the exchange of gases (oxygen $\Leftrightarrow$ carbon dioxide) with the environment. Air enters the body via the upper respiratory tract (nose, mouth and throat) and gets into the lung - the lower respiratory tract. Beginning with the trachea, the lower respiratory tract branches at the main bronchus into the left and right lung harboring two and three lobes, respectively. From the hilus, the lobar intrapulmonary bronchi then branch further into terminal bronchioli, respiratory bronchioli, alveolar ducts and end in the alveolar sacs (figure 1). The tracheobronchial tree including the terminal bronchiole is considered as the air conducting part, whereas the part distal is considered as the respiratory part, which represents a gas exchange area of 50-100 $\mathrm{m}^{2}$ in humans (84).

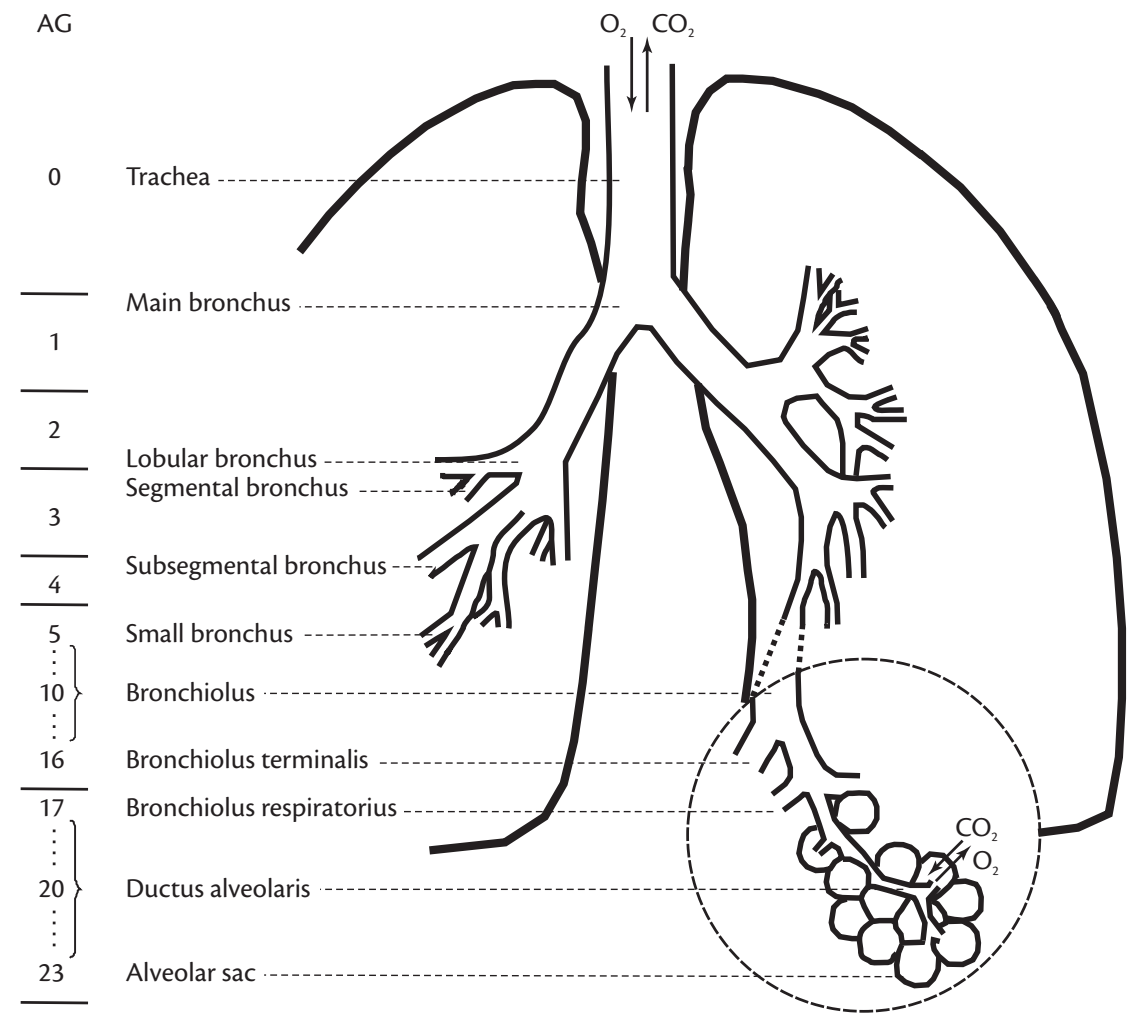

Figure 1: The tracheobronchial tree of the human lung. Air rich in oxygen $\left(\mathrm{O}_{2}\right)$ enters the lung via the trachea and is conducted by the airways down to the alveoli, where the gas exchange against carbon dioxide $\left(\mathrm{CO}_{2}\right)$ from blood takes place. On the left, the airway generation (AG) refering to its according anatomical structure is depicted.. The figure was modified according to Klinke and colleagues (84)..

Mammalian tracheobronchial branching architectures are complicated and cannot be idealized to one branching system. Branching of conducting airways in humans is considerably asymmetrical and asymmetry may have an important effect on airflow. Three idealized branching patterns are commonly recognized for different species: (i) monopodial (at the branching point a small segment may branch from the main, or parent stem), (ii) dichotomous (the parent segment may divide into 
two equal daughter segments) and (iii) polychotomous (the parent segment may divide into many daughter segments) (83).

A cross-section of a conducting airway reveals a concentric anatomic structure (figure 2). The airway lumen is faced by a single cell layer, the epithelium, lying on the basal membrane. The airway smooth muscle (ASM) cells form a thin bundle around the airway. The purpose of ASM in the airways remains contentious, but certainly contraction of ASM is responsible for the excessive airway narrowing observed in asthma (77). In humans, ASM are found down to the respiratory bronchioles, whereas cartilage is only interspersed in the conducting airways.

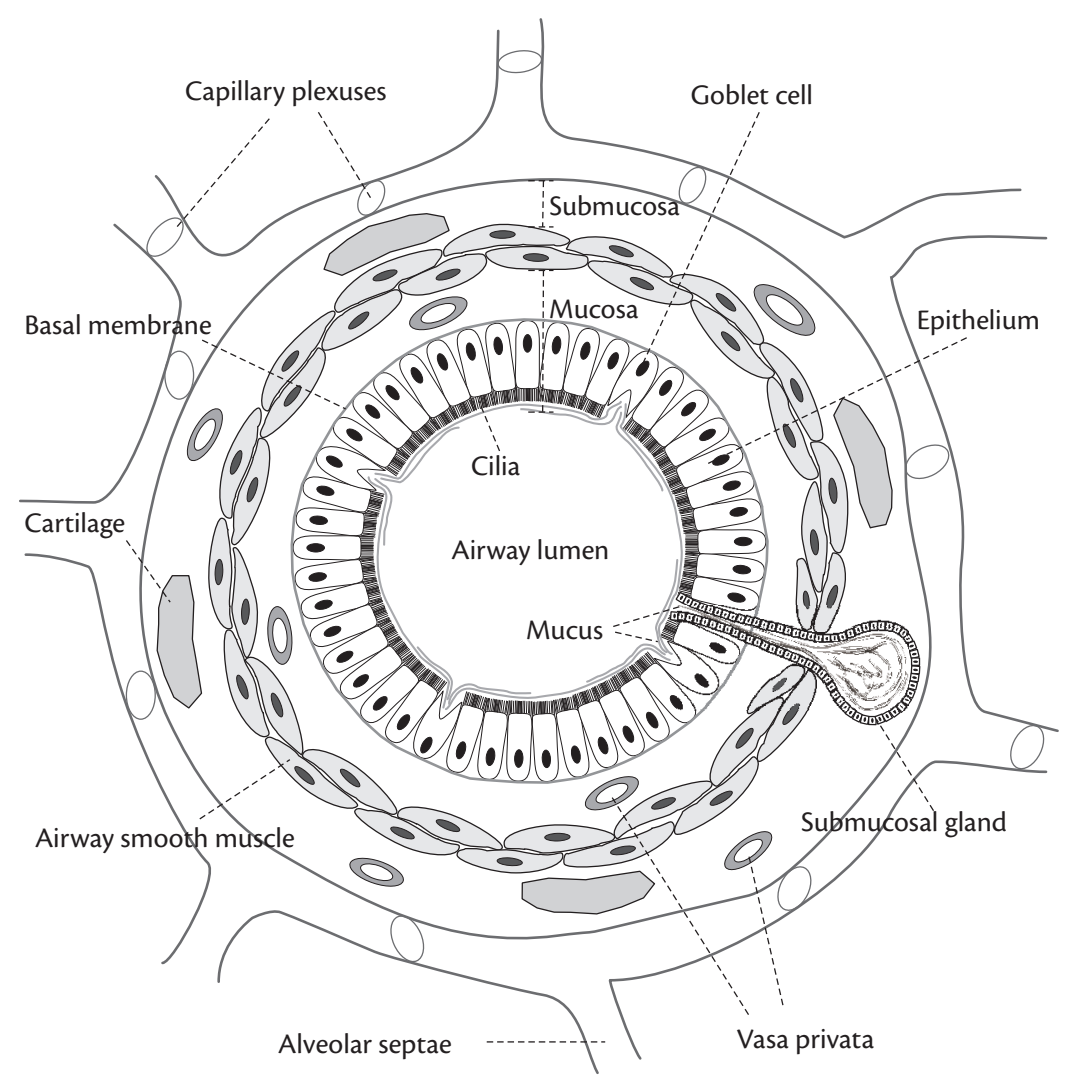

Figure 2: Cross-section of an airway. The concentric structure possesses a single cell layer - the epithelium which is ciliated - facing the airway lumen and lying on the basal membrane. A thin bundle of airway smooth muscle cells follows. The vasa privata provide the lung with blood, whereas the gas exchange of oxygen and carbon dioxide takes place at capillary plexuses. Tethering forces are conducted by the alveolar septae. For more details please see the text.

Removal of particles or microorganisms from the lung is achieved by mucociliary clearance, i.e. the mucus - produced by goblet cells or submucosal glands - forms a continuous layer, to which the foreign bodies stick, that is transported by the beating cilia towards the upper respiratory tract (84). Cilia and mucus are not found in the alveoli as they would impair the gas exchange. At this site, protection against foreign particles is achieved by alveolar macrophages. They phagocytize the foreign particles and enzymatically degrade them or, if this is not possible, the macrophages migrate ameboidly towards the conducting airways and are then removed by mucociliary clearance (84). 
The vasa privata originate from the systemic circulation and provide the lung with blood. In contrast in the pulmonary circulation, the pulmonary artery and vein branch out and form capillary plexuses at alveolar sites where gas exchange takes place between air and blood (129). Tethering forces of the parenchyma, pulmonary pressure and partially also cartilage keep the airways in an open uncollapsed state. The parenchyma consists of alveolar ducts, alveolar sacs, alveoli and the airspaces contained within these structures. In the inflated lung the parenchyma constitutes approximately $80-90 \%$ of the lung volume and consists of around $70 \%$ airspace and $30 \%$ gas exchange tissue which surrounds the airspace (106).

\subsection{Autonomic innervation of the lung}

The mammalian nervous system is divided into the central- and peripheral nervous system. The central nervous system is comprised of the brain and the spinal cord, whereas the peripheral nervous system consists of all somatic and autonomous nerves including ganglions. The somatic nervous system mediates between the organism and its environment. It is associated with conscious perception, voluntary body movements via skeletal muscles and rapid signalling. In contrast, the autonomous nervous system mediates between the organism and its organs. It is responsible for body homeostasis and the regulation of organ functions depending on the environmental conditions. It functions unconsciously and involuntarily. Both, the somatic and the autonomic nervous system, contain afferences and efferences (figure 3). Afferences are all neural pathways originating in the periphery (e.g. skin, organs) and running to the central nervous

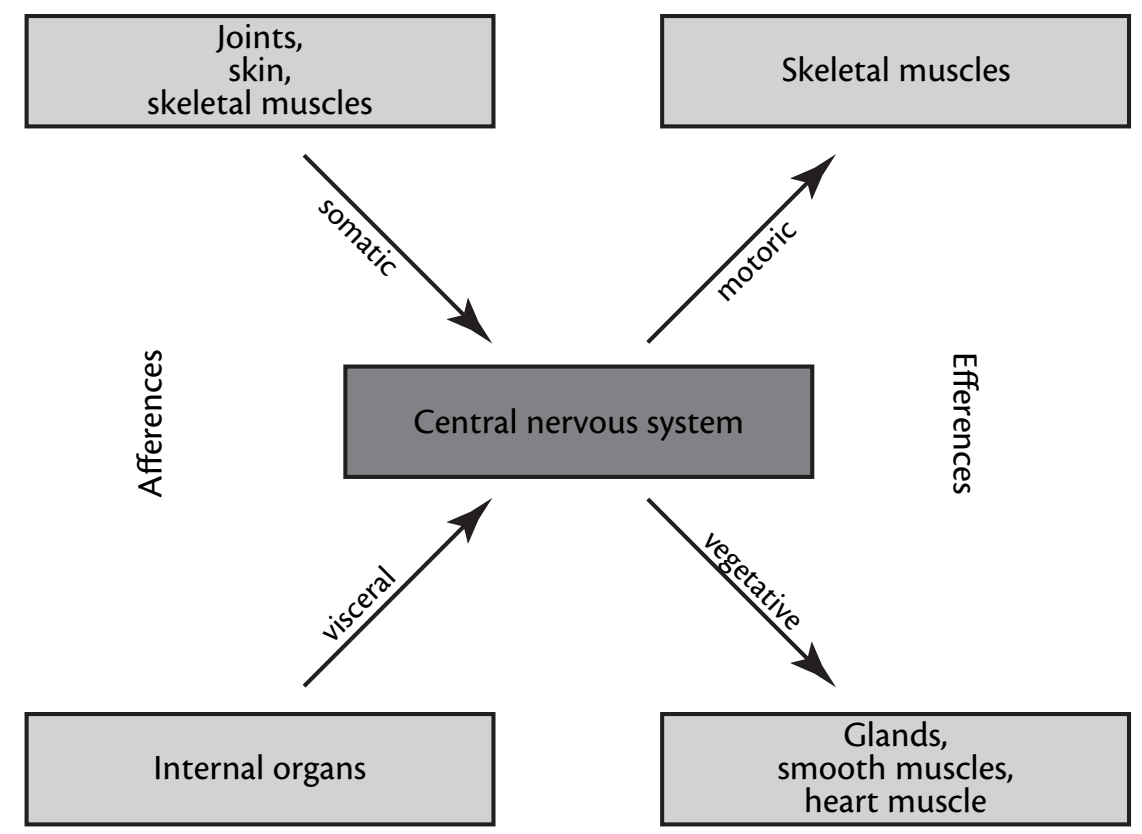

Figure 3: Interconnections between the peripheral and central nervous system. The peripheral nervous system (light grey) contains both afferences and efferences. Signals originating in the periphery are conducted via the afferences to the central nervous system (brain + spinal cord; dark grey), become integrated and are then fed back to the periphery via efferent nerves resulting in an adaptation of the organism to the stimulus. The upper panels reflect the somatic nervous system and the lower panels the autonomous nervous system. 
system. By contrast, efferences are all neural pathways running from the central nervous system to the periphery (e.g. voluntary muscles, smooth muscles, vessels or glands) (51).

The autonomous nervous system is further subdivided into the sympathetic and parasympathetic nervous system as well as the enteric nervous system innervating the intestinal wall. Usually, an organ is innervated by both sympathetic and parasympathetic nerves antagonizing each other. Sympathetic nerve fibers are associated with the spinal cord (thoracic vertebrae 1-12 and lumbar vertebrae 1-4), whereas parasympathetic nerve fibers originate from the brain stem and the sacral vertebrae 2-4 (51). In the lung, sympathetic fibers are associated with the thoracic vertebrae 1-5 and parasympathetic fibers with the $10^{\text {th }}$ cranial nerve (Nervus vagus) (129). With respect to the neurotransmitter involved at the neuromuscular synapse there exist three types of nerves: sympathetic noradrenergic nerves, parasympathetic cholinergic nerves and nonadrenergic noncholinergic (NANC) nerves $(9 ; 32 ; 49)$. Moreover, nerve fibers are distinguished by their conduction velocities into $C-(0.3-2 \mathrm{~m} / \mathrm{s}), A \delta-(3-8 \mathrm{~m} / \mathrm{s})$ and $A \delta / \gamma$-fibers $(10-50 \mathrm{~m} / \mathrm{s})(97 ; 174)$.

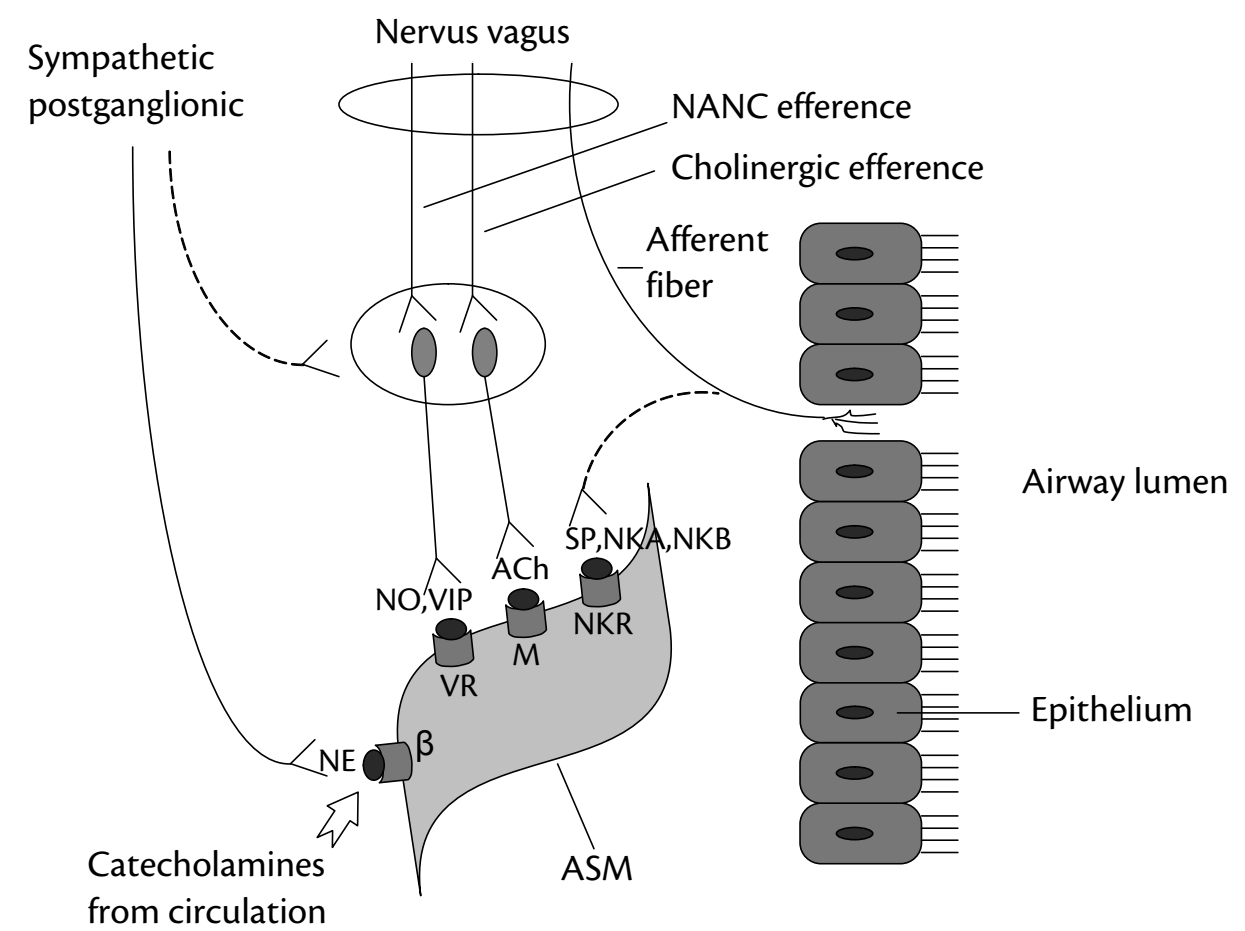

Figure 4: Innervation of the airway smooth muscle (ASM). The ASM is innervated by cholinergic, adrenergic and nonadrenergic noncholinergic nerves (NANC) (9; 142). Excitatory and inhibitory components run side by side, in which excitatory mechanisms lead to ASM contraction and inhibitory mechanisms to ASM relaxation. The excitatory cholinergic innervation is present in the airways from the trachea down to the peripheral bronchi. Bronchoconstriction via excitatory NANC is mediated via subepithelial C-fiber sensory afferents that release tachykinins (neurokinin (NK) A and B, and substance P (SP)). Next to the noradrenergic inhibitory system originating from the adrenal medulla or sympathetic ganglia, inhibitory NANC innervation has been confirmed, in which vasoactive intestinal polypeptide (VIP) and nitric oxide (NO; diffusing through ASM membrane and activating intracellular soluble guanylyl cyclase) are involved as neurotransmitters. Except for the cholinergic nerve component which seems to be present in all mammals, the innervations of the ASM show a considerable variability among different species. $A C h$, acetylcholine; $\beta$, $\beta$-adrenoceptor; $M$, muscarinic acetylcholine receptor; NE, norepinephrine; NKR, NK receptor; VR, VIP receptor. The figure was modified according to Andersson and Grundström (9). 
The autonomic nerves regulate many aspects of airway function, e.g. ASM tone, mucus secretion and bronchial microcirculation (143). Upon activation, the nerves release their inherent neurotransmitters, which then have a post-junctional effect on their end organ. With respect to the ASM, neurotransmitters initiate bronchoconstriction or relaxation (figure 4). Bronchoconstrictive neurotransmitters are for instance acetylcholine, which is released by parasympathetic cholinergic nerves, and the tachykinins substance $P(S P)$, neuropeptide $K$, neurokinin (NK) A or B, which are released from excitatory NANC (eNANC). ASM relaxation is caused by $\beta_{2}$-receptor stimulation after activation of sympathetic adrenergic nerves or by the release of vasoactive intestinal polypeptide (VIP) or nitric oxide (NO) from inhibitory NANC (iNANC) $(9 ; 59)$.

Innervation of the ASM differs between species as well as within species along the tracheobronchial tree. Spina and colleagues (158) studied the sensory nerve functions of different species and found that the relative tissue content of the neuropeptides calcitonin gene related peptide (CGRP) and SP was much higher for guinea pigs than for rabbits and marmosets, which had equal levels. In some cases the same neuropeptides can even have opposing effects: Whereas SP or NK A cause relaxation of tracheal preparations from Sprague-Dawley and Wistar rats, they lead to contraction of tracheal preparations from Fischer 344 rats $(42 ; 56 ; 81 ; 165)$. Within guinea pigs, cholinergic nerves dominate over tachykinergic at the trachea, whereas tachykinergic nerves dominate at the bronchus (8). The human lung contains ASM constrictive cholinergic and eNANC nerves, and ASM relaxation is mainly due to iNANC nerves whereas sympathetic adrenergic nerves may play only a minor role (32).

\subsection{Mechanisms of neurally induced ASM contraction}

The parasympathetic nervous system is the dominant bronchoconstrictor mechanism in all mammals (14). Acetylcholine is released from nerve terminals and binds to muscarinic ( $M$ ) receptors at different sites throughout the lungs. The $M_{1}$ receptor is localized on parasympathetic ganglia and is thought to primarily facilitate cholinergic neurotransmission by nicotinic acetylcholine receptors. $M_{2}$ receptors on the pre-synaptic nerve terminals control acetylcholine release in a feedback manner, limiting excessive acetylcholine liberation. Moreover, $M_{2}$ and $M_{3}$ receptors are found on the ASM cells. $M_{3}$ receptor activation leads to ASM contraction, a process facilitated by $M_{2}$ receptors by counteracting CAMP-mediated relaxant pathways (21). Regarding eNANC innervation released tachykinins bind to NK receptors located on the ASM. SP binds principally to the $\mathrm{NK}_{1}$ receptor, whereas NK $\mathrm{A}$ and $\mathrm{NK} \mathrm{B}$ bind to $\mathrm{NK}_{2}$ and $\mathrm{NK}_{3}$ receptors, respectively (6). Both, $M$ and NK receptors belong to the family of heterotrimeric guanine nucleotide-binding protein (G protein) coupled receptors (GPCR). $\mathrm{M}_{3}$ and $\mathrm{NK}_{1-3}$ preferentially couple to the $\mathrm{G}_{\mathrm{q} / 11}$ protein $(6 ; 21)$. Receptor stimulation results in dissociation of the $G$ protein and subsequent activation of the phospholipase $C$ by $G_{\beta \gamma}$. Phospholipase $C$ hydrolyzes phosphatidylinositol-4,5-bisphosphate to diacylglycerol (DAG) and inositol $(1,4,5)$-trisphosphate $\left(\mathbb{P}_{3}\right)(5)$. Then $\mathbb{P}_{3}$ binds to the $\mathbb{I}_{3}$ receptor at 
the sarcoplasmic reticulum (SR) causing the release of calcium from intracellular stores (115) and resulting in a 10000-fold increase in intracellular calcium concentration (72). In a resting (relaxed) ASM cell the intracellular free calcium ion concentration is approximately 100-200 nM (147). The elevated intracellular calcium concentration causes the formation of the calcium/calmodulin complex, which activates the myosin light chain kinase (MLCK). The contractile apparatus of the ASM consists of actin and myosin filaments and accessory and regulatory proteins. Phosphorylation of the myosin light chain by MLCK enhances its intrinsic adenosine triphosphatase activity and subsequent mechanical interactions with actin. Finally, this results in the sliding of the myosin molecule along the actin filament (72).

ASM contraction can also occur after increases of cytosolic calcium concentration due to calcium entry from extracellular sites via voltage gated calcium channels, the reverse mode of the sodium calcium exchanger (NCX), voltage independent influx via transient receptor potential (TRP) channels or activation of the ryanodine receptor at the SR (72; 115; 147). However, these mechanisms are beyond the scope of neurally induced ASM contraction and are therefore not explained in detail here.

Another mechanism leading to ASM contraction operates by the inhibition of the myosin light chain phophatase (MLCP). Thereby, the dephoshorylation of the myosin light chain is prevented and ASM contraction is promoted. Inhibition of MLCP is due to activation of the Rho-kinase pathway in response to $G_{12 / 13}$ stimulation $(62 ; 186)$ or due to $D A G$ formation after $G_{q / 11}$ stimulation. DAG activates the protein kinase C (PKC) (5). PKC in turn phosphorylates the following protein PKC activated phosphatase inhibitor (CPI)-17, which functions as inhibitor of the catalytic subunit of $\operatorname{MLCP}(62 ; 186)$.

Phamacologically, ASM contraction can be inhibited by receptor antagonists which is also of clinical relevance, e.g. tiotropium in chronic obstructive pulmonary disease (COPD) (134). A variety of anticholinergics, such as atropine, 4-DAMP, AQ-RA, gallamine, DAU 5884, methoctramine, pirenzepine, AF-DX 116, ipratropium or tiotropium have been developed and show different specificity concerning the $M$ receptor subtypes (62). Selective antagonists of the NK receptors are for instance aprepitant, SR140333, LY303870, CP99994, and RP67580 for NK ${ }_{1}$, GR94800, GR159897,

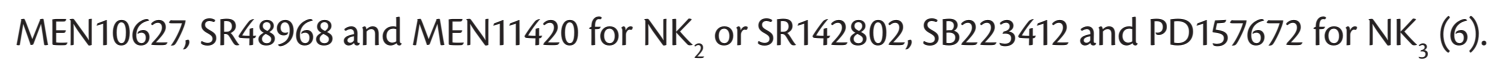

\subsection{Mechanisms of neurally induced ASM relaxation}

ASM relaxation is caused either by sympathetic or iNANC signalling. In sympathetic signalling, norepinephrine functions as neurotransmitter binding to $\beta$-receptors on the ASM (19). In iNANC signalling, VIP or NO are involved as neurotransmitters binding to the VIP receptor on ASM or interfering with the cyclic guanosine monophosphate (cGMP) pathway in ASM, respectively 
(22; 101; 116; 178). Both, the $\beta$-receptor and the VIP-receptor belong to the family of GPCRs coupling to the $G_{s}$ protein (6). Upon dissociation of $G_{s}$, in response to receptor stimulation, the membrane bound adenylyl cyclase becomes activated catalyzing the formation of cyclic adenosine monophosphate (CAMP) from adenosine triphosphate (ATP) $(5 ; 20 ; 98)$. Increased CAMP levels are responsible for ASM relaxation due to several mechanisms: On the one hand, CAMP may promote the efflux of calcium to the extracellular space via NCX or via calcium-ATPases into the SR. On the other hand CAMP may activate the protein kinase A (PKA) (85). The PKA leads to phosphorylation of the MLCK preventing MLC phosphorylation (58) or PKA attenuates RhoA phosphorylation (92), which decreases Rho association with its downstream target Rho kinase (44).

NO activates the soluble guanylyl cyclase forming cGMP, which activates the protein kinase $G$ (PKG). PKG causes smooth-muscle relaxation by mechanisms that are still being defined and that include reduction in cytosolic calcium (by enhanced calcium export and/or by reduction in $\mathrm{IP}_{3}$ receptor-mediated calcium-mobilization) and dephosphorylation of MLC (by activation of MLCP and/or by sequestration of MLCK in a phosphorylated form, which is inaccessible for calcium/ calmodulin complexes) (20).

ASM relaxation can be influenced pharmacologically. With respect to $\beta$-receptors, a variety of antagonists and agonists are established. Antagonists are for instance the $\beta$-blockers, propranolol and arterenol. Agonists have clinical relevance in the treatment of asthma and are for instance salbutamol, isoproterenol, indacaterol, salmeterol or formoterol, which are classified as short or long acting $\beta$-agonists (SABA/LABA) depending on their duration of action (125). Moreover, direct blocking of catecholamine release from adrenergic nerves can be achieved by the application of guanethidine (52). Small peptides resembling the growth hormone releasing factor are considered as antagonists at the VIP-receptor $(6 ; 63 ; 182)$. NO-signalling is antagonized by $\mathrm{NO}$ synthase inhibitors such as $\mathrm{N}_{\omega}$-nitro-L-arginine methyl ester (L-NAME) (139) or $\mathrm{N}_{\omega}$-nitro-L-arginine (L-NNA) (102; 103).

\subsection{Electric field stimulation (EFS) of lung tissue preparations in organ baths}

Application of an electric field across isolated airway preparations results in activation of intrinsic nerves, the release of neurotransmitters, and response of the ASM to these neurotransmitters (52). Previously, EFS has been studied in three types of airway preparations, i.e. tracheal strips, bronchial strips and parenchymal strips (52), which were mounted in organ baths and faced by parallel platinum electrodes. The electrodes can be wire or flat stock placed parallel to the preparation or ring electrodes placed at each end of the tissue (52). These models have been extremely useful for understanding the neural control of airway tone in different species e.g. mouse, rat, guinea pig, ferret, cat, dog, horse or human $(8 ; 11 ; 49 ; 57 ; 61 ; 71 ; 80 ; 93 ; 104 ; 125 ; 183 ; 184 ; 190)$, and continues to do so $(25 ; 125)$. However, the organ bath method has some disadvantages: First, it can be applied 
only to relatively large airways and preparations from the lower airways of smaller mammals are difficult to obtain. For example in rats, studies of the innervation are characterized only down to the eighth generation (52). However, innervation is unevenly distributed along the tracheobronchial tree. For instance, in guinea pig trachea cholinergic nerves predominate over tachykinergic nerves, whereas the latter predominate in the bronchus (8). In vivo parasympathetic vagal stimulation and tantalum roentgenography showed a higher bronchospasm in intermediately sized airways compared to larger airways in dogs or cats. Therefore, methods to examine smaller sized airways in different species are needed (124). Second, organ bath methods are often performed under either isometric or isotonic conditions and require a muscle preload. This creates potential problems: the preload is artificial and may influence the result or even damage the smooth muscle (52). Furthermore, contractions are not auxotonic as in vivo, where the ASM shortens against an increasing load imposed by the attachments of the surrounding lung parenchyma, parallel elastic elements (i.e. extracellular matrix) and mucosal folding (34; 52; 96). More recently, some of these problems have been solved by the development of servo-controlled force-length transducers that adjust airway preparations to match a muscle length that was determined before the specimen was prepared $(25 ; 26)$. Third, the smaller the airways are the smaller is the amount of smooth muscle and quantification of weak contractions requires very sensitive equipment. Spiral tissue preparations, used to increase contractions, are hampered by misalignment of the smooth muscle from the longitudinal axis and force generation is lost to the coil (52). Fourth, organ bath experiments require a relatively large buffer volume, generally more than $5 \mathrm{~mL}$. This is disadvantageous, if expensive substances have to be tested and/or a high throughput of experiments is intended. Fifth, in whole tissue preparations (i.e. parenchymal strips) contractions from airways or vessels cannot be distinguished (52).

\subsection{Precision-cut lung slices (PCLS)}

PCLS are a relatively new in vitro method in pulmonary research. PCLS are lung tissue slices of uniform thickness of $200-300 \mu \mathrm{m}$, which are prepared from agarose instilled lungs by means of a Krumdick tissue slicer and which are viable for at least $72 \mathrm{~h}$ under cell culture conditions $(111 ; 159)$. In 1996, Martin and colleagues (111) described the preparation and study of airway responses in PCLS for the first time. Originally developed for rats, the PCLS method has been adopted to other species such as mice (34; 68), guinea pigs (145), horses (181), sheep (151), non-human primates (155) and humans $(145 ; 163 ; 188)$. Depending on the species 10 to more than 50 slices can be obtained from one animal. Therefore, this method is very economical in terms of experimental costs and animal use, thus contributing to Russell's and Burch's (149) 3Rs (refinement, reduction, replacement) of animal protection: The PCLS Method has the potential to (i) refine the multiple physiological measurements, (ii) reduce the number of animals by a factor of 10 to 50 and (iii) replace animals when human lung tissue is used (110). 
PCLS have been extremely useful in pharmacological $(69 ; 107 ; 145 ; 152 ; 188 ; 189)$ and toxicological studies $(68 ; 127 ; 128)$ because they resemble the intact respiratory tract of the in vivo situation in morphology and functionality. Some advantages of the PCLS method are that airway contractions are somehow auxotonic as the airways are embedded in the surrounding tissue (34), that contractions of vessels and airways can be distinguished (108), that only small buffer volumes are needed, that many slices can be obtained from an individual so that different conditions can be tested at the same subject and that small airways are accessible allowing to study the complete tracheobronchial tree down to the peripheral lung $(111 ; 152 ; 189)$. Even biochemical analysis can be performed on PCLS, i.e. gene expression profiles can be analysed after reverse transcription polymerase chain reaction (38) or intracellular calcium fluxes can be measured in multiphoton microscopy $(39 ; 145)$.

Small airways are defined by their inner diameter being less than $2 \mathrm{~mm}$ in human and less than $780 \mu \mathrm{m}$ in rat airways $(189 ; 191)$. As a result of their relative inaccessibility, the role of small airways is difficult to assess and maybe because of these problems small airways have been considered as a silent zone in the past. Since in PCLS the airway diameter is easily accessible, this method offers a novel way to study airway responses in peripheral airways (110). The smallest airways being analyzed by Wohlsen and colleagues (189) in PCLS from rat were of $50 \mu \mathrm{m}$ in diameter, corresponding roughly to the terminal bronchioles. Bronchoconstriction in PCLS has been studied in response to exogenously administered mediators of the early allergic response (e.g., methacholine, histamine, serotonin, thromboxane (TXA), leukotriene (LT), endothelin) and in response to allergens after passive or active sensitization (34; 189). Smaller airways in PCLS are more reactive to methacholine, serotonin, the thromboxane prostanoid (TP) receptor agonist U46619 and allergens in lungs of different species (112; 188; 189). Different explanations for these longitudinal differences in airway responsiveness are possible: for instance, heterogeneous receptor distribution along the airways, as shown for VIP (33; 137), tachykinin (55), SP (132), and histamine (28) or varying ASM thickness (thickness is more pronounced in small than in large airways) (48), which might result in differences in airway reactivity $(120 ; 162)$. This last argument is contradicted by findings that endothelin contracted airways independent of their size (112), while small airways react stronger to methacholine and TXA $(111 ; 112)$. Given the importance of small airways for asthma and COPD $(160 ; 188 ; 189)$, the possibility to study different airway generations in PCLS is of clinical relevance.

Mucociliary clearance is an important defense mechanism that clears foreign particles and chemicals from the airways to maintain healthy lungs. A key parameter determining the rate of mucus clearance is the ciliary beat frequency (CBF). The density (cilia per cell and number of ciliated cells) and length of cilia are greater in the trachea than in small airways and this correlates with an increase in mucus transport and CBF toward the oropharynx (41). Hence, beside bronchoand vasoconstriction, measuring the CBF has been addressed in PCLS from mice, rats and humans (41; 187; 188). CBF in those species varied from $23.1-26.5 \mathrm{~Hz}$ in mice to $12.7-15.6 \mathrm{~Hz}$ in rats and 
$4.8-12.9 \mathrm{~Hz}$ in humans. Although species variation may occur, PCLS serve as a suitable model to examine regulation of $\mathrm{CBF}$ in response to physiological parameters (e.g. temperature) (41) or drug interference (e.g. CAMP elevating agents such as roflumilast), which have shown beneficial effects in COPD or asthma (187).

Overall, as results obtained from PCLS are predictable for responses of the entire intact lung (110), PCLS are of great use in pulmonary toxicology and pharmacology, i.e. development of drugs for asthma and COPD. Certainly, human PCLS provide a unique opportunity to save animals and to get clinically relevant data at the same time.

\subsection{Asthma and COPD}

Bronchial asthma and COPD have a high personal as well as societal, and economical impact (177). Chronic lower respiratory tract diseases are the fourth-leading cause of death and the major cause of sickness in the United States (123). Worldwide, they are currently the fifth-leading cause of death and the World Health Organization projects they will become the third-leading cause by 2030 (154). Therefore, it is critical to understand their underlying pathogenic mechanisms in order to develop new treatment strategies.

Both asthma and COPD are characterized by airway obstruction, which is variable and reversible in asthma but is progressive and largely irreversible in COPD. In asthma, airway obstruction is mainly due to bronchoconstriction, edema and plasma exudation, whereas in COPD mucosal inflammation, fibrosis, emphysema and mucus hypersecretion are responsible for obstruction (19). In both diseases, the respiratory tract is chronically inflammed (table 1), which is mediated by increased production of multiple inflammatory proteins, including cytokines, chemokines, adhesion molecules, inflammatory enzymes and receptors (19). Moreover, acute episodes of exacerbation are observed with increasing intensity of inflammation in both diseases (19). Ultimately, chronic inflammation leads to airway remodelling which affects the whole respiratory tract from central to peripheral airways that are less than $2 \mathrm{~mm}$ in internal diameter in humans, the so called small airways (177).

Asthma is an allergic disease, in which mast cells become activated and initiate the early allergic response (EAR) within minutes and the late allergic response (LAR) within hours leading to the typical asthma symptoms: wheezing, coughing, chest tightness and shortness of breathing (170). In the EAR, inhaled allergens activate mast cells, by cross-linking surface bound immunoglobulin (Ig) E, that degranulate and sequester preformed (histamine, proteoglycans, serine proteases, tumor necrosis factor (TNF)) or newly synthesized mediators (prostaglandins (PG), cysteinyl LT, TXA, platelet activating factor, cytokines). These mediators interact and lead to bronchoconstriction (17; 18; 170). The LAR appears frequently $4-6$ hours after the EAR and 
depends on leukocyte (eosinophils, mast cells and T-lymphocytes) recruitment due to mast cellderived mediators such as TNF- $\alpha$ and different chemokines as well as the expression of adhesion molecules on leukocytes and vascular endothelial cells (170). Those leukocytes initiate the second phase of bronchoconstriction, mucus secretion and airway swelling. Moreover, they cause tissue destruction, launch and maintain the chronic inflammation (170). T helper lymphocytes type 2 ( $\mathrm{T}_{\mathrm{H}}$ 2-lymphocytes) have a central role in regulating the inflammatory response in allergy through the release of interleukins (IL). IL-4 and IL-13, stimulate B-lymphocytes to produce IgE, IL-5 attracts eosinophils and IL-9 stimulates mast-cell proliferation (18).

Table 1: Characteristics of asthma and COPD in chronic inflammation of the lower respiratory tract (modified from Barnes $(17 ; 18)$ )

\begin{tabular}{|c|c|c|}
\hline Character & Asthma & COPD \\
\hline Airway smooth muscle & Increased & Minimal increase \\
\hline Basement membrane & Thickened & No change \\
\hline Fibrosis & Subepithelial & Peribronchiolar \\
\hline Alveolar disruption & No & Existent \\
\hline Airway vessels & Increased & No change \\
\hline Epithelium & Often shed & Pseudostratified \\
\hline Goblet cells & Increased & Increased \\
\hline Mast cells & Increased and activated & Normal \\
\hline Eosinophils & Increased & Normal \\
\hline Neutrophils & Normal (severe: increased) & Increased \\
\hline Dendritic cells & Increased & Uncertain \\
\hline T lymphocytes & $\mathrm{T}_{\mathrm{H}} 2$ type & $T_{H} 1$ and $T_{C} 1$ type \\
\hline B lymphocytes & IgE producing & Increased \\
\hline Cytokines & IL-4, IL-5, IL-13, TNF- $\alpha$ & TNF- $\alpha$ \\
\hline Chemokines & CCL11, CXCL8 & CCL2, CXCL1, CXCL8 \\
\hline Lipid mediators & $\mathrm{LTD}_{4}, \mathrm{LTB}_{4}, \mathrm{PGD}_{2}$ & $\mathrm{LTB}_{4}$ \\
\hline Oxidative stress & Yes & Yes \\
\hline Steroid response & Sensitive & Resistant \\
\hline
\end{tabular}

CCL, CC-chemokine ligand; COPD, chronic obstructive pulmonary disease; CXCL, CXC-chemokine ligand; IL, interleukin; $\mathrm{LTB}_{4^{\prime}}$ leukotriene $\mathrm{B}_{4^{\prime}} \mathrm{LTD}_{4^{\prime}}$ leukotriene $\mathrm{D}_{4^{\prime}} ; \mathrm{PGD}_{2^{\prime}}$, prostaglandin $\mathrm{D}_{2} ; \mathrm{T}_{C} 1$, type 1 cytotoxic $T$ cell; $\mathrm{T}_{H^{\prime}}$ Thelper cell; TNF, tumor necrosis factor.

In contrast to asthma, in COPD, inhaled cigarette smoke and other irritants activate epithelial cells and macrophages to release chemotactic factors that attract monocytes (differentiating into macrophages in the lung), neutrophils, $T$ helper lymphocytes type 1 ( $T_{H} 1$-lymphocytes) and type 1 cytotoxic $T$ cells ( $T_{c} 1$-cells) (18). These cells, together with macrophages and epithelial cells, release proteases, e.g. matrix metalloproteinase 9 causing elastin degradation and emphysema. Neutrophil elastase also causes mucus hypersecretion. Epithelial cells and macrophages release transforming growth factor- $\beta$, which stimulates fibroblast proliferation, resulting in fibrosis in the small airways (18). 


\section{Airway hyperresponsiveness (AHR) and airway remodelling in asthma}

AHR is defined as an exaggerated airway narrowing in response to many different stimuli, e.g. histamine, methacholine or adenosine $(15 ; 170)$. In everyday life, increased bronchial responsiveness may occur following allergen exposure, upper respiratory tract viral infection or industrial chemical contact (15). Depending on the trigger, various types of asthma exist, that are for example extrinsic (allergic) asthma, intrinsic asthma, exercise-induced asthma, aspirin-sensitive asthma and occupational asthma $(35 ; 64)$.

Airway remodelling is a consequence of chronic airway inflammation and refers to structural changes in the lung. These are thickening of the airway wall, hyperplasia of goblet cells, hypertrophy of submucosal glands, increased vascularity of the airway wall, ASM hypertrophy, ASM hyperplasia and subepithelial fibrosis (table 1$)(13 ; 170)$. The airway wall thickening may explain incomplete reversibility of airway obstruction in asthmatics, whereas altered goblet cells and submucosal glands explain mucus hypersecretion and mucus plugging (170). Moreover, the increased vascularity is responsible for amplified plasma protein leakage into the lung (170). The neural function is also influenced in asthma and a separate chapter is dedicated to that topic (chapter 1.9).

\subsection{Animal models of asthma}

As a consequence of the continuous increase in prevalence and morbidity in asthma, there is an urgent need to develop new drugs for therapy. In general, animal models provide a surrogate for humans to test hypotheses concerning a disease. The strengths of animal models include: (i) being genetically homogenous and well defined study subjects; (ii) the ability to use invasive, in vivo, experimental methods to identify potential mechanisms; (iii) the accessibility of tissue for in vitro studies; (iv) the ability to control and manipulate environmental and/or genetic factors; and ( $v$ ) the ability to develop and test pharmaceuticals for human use (1). With regard to allergic asthma, an ideal animal model should develop first an EAR, second a LAR, third non-specific AHR to various stimuli, forth eosinophilic and neutrophilic bronchial inflammation, fifth changes in mucus secretion and quality, sixth alterations in airway and lung histology and seventh spontaneous increase in resistance of airways after prolonged or repeated allergen exposure (83; 166). However, so far there is no animal known with a natural disease course perfectly mimicking human asthma and no animal model is available that completely reproduces the multiple features of human asthma (table 2) (83). Hence, all established models are approaches to reflect particular aspects of human asthma.

Animal models of asthma are distinguished into three groups: (i) animals having naturally occurring recurrent airway obstruction, e.g. cats (idiopathic asthma: wheezing, cough and cyanosis) $(1 ; 83)$ and horses (heaves) (1), (ii) animals having an AHR before any provocation in the laboratory 
Table 2: Asthma symptoms in selected animal models compared to human asthma (referring to $(1 ; 83$; 144))

\begin{tabular}{|c|c|c|c|c|c|c|c|c|c|c|c|}
\hline Symptom & Mouse & Rat & $\begin{array}{l}\text { Guinea } \\
\text { pig }\end{array}$ & Rabbit & Cat & Dog & Pig & Sheep & Horse & Monkey & Human \\
\hline $\begin{array}{l}\text { Naturally } \\
\text { sensitive }\end{array}$ & - & - & - & - & $+/-$ & + & - & + & + & + & + \\
\hline EAR & - & + & + & $+/-$ & + & + & $+/-$ & + & - & $+1-$ & + \\
\hline LAR & + & + & + & + & - & + & + & + & - & + & + \\
\hline AHR & + & $+/-$ & + & + & + & + & - & + & + & + & + \\
\hline Inflammation & + & + & + & $+1-$ & + & + & + & + & + & + & + \\
\hline Eosinophilia & + & + & + & - & + & - & + & + & - & + & + \\
\hline Neutrophilia & - & - & - & - & - & + & + & - & + & - & + \\
\hline Remodelling & - & + & - & - & + & + & - & + & - & - & + \\
\hline
\end{tabular}

$E A R$, early allergic response; $L A R$, late allergic response; $A H R$, airway hyperresponsiveness

occurs (73; 135) and (iii) animals developing reversible airway obstruction and/or increased airway responsiveness only after challenge, among which are small laboratory animals (e.g mouse, rat and guinea pig) (144) but also large animals (e.g. sheep). Remarkably, sheep may also develop a natural sensitivity, when they encounter the allergen Ascaris suum in their natural environment (2) and the use of an anthelminthic is therefore required during sensitization of sheep with for instance house dust mite (HDM) (24).

Concerning the latter of the three groups, sensitization of the animal is a prerequisite for studying airway responses. In general, animals are sensitized with an allergen, which is commonly ovalbumin (OVA) $(60 ; 75 ; 91 ; 117 ; 185)$ or HDM $(23 ; 24 ; 27 ; 157)$, by repeated allergen challenge over days to weeks to establish an adaptive immune response by the animal. Many sensitization protocols have been developed in which the allergen is applied either intraperitoneally, subcutaneously, intratracheally/intralobularly or by aerosolization. In some cases, an adjuvant such as aluminium hydroxide or heat inactivated Bordetella pertussis organisms may be required to induce $\mathrm{T}_{\mathrm{H}}$ 2-lymphocyte driven immune responses and ultimately the production of IgE (87).

The rat exhibits many features of airway allergy and allergic asthma that are similar to those of humans (table 2) (87). Therefore, the ability to extrapolate to humans is one of the advantages of this species. Similarities between rats and humans include (i) EAR and LAR after an allergen challenge, (ii) responses after a nonspecific challenge with methacholine, acetylcholine, or serotonin, (iii) IgE production and (iv) an accumulation of inflammatory cells (87). The ability to produce EAR, LAR and AHR makes the rat favorable over the mouse model (87), which is advantageous when genetic modifications or gene knock-outs are required to study the involvement of distinct endogenous proteins in the pathomechanisms of asthma (94). AHR, airway inflammation and obstruction can be easily reproduced in rats, although this may depend on the rat strain: Wistar rats are prone to 
sensitization with OVA, producing similar but less pronounced effects compared to those observed in Brown Norway rats (87). In contrast, Sprague Dawley rats do not develop an allergic reaction or an increase in IgE production under the same conditions and usually serve as a control group for Brown Norway rats (87). Singh and colleagues (157) studied the allergic airway response in three rat strains in response to HDM and found that Brown Norway rats develop strong and Sprague Dawley rats moderate hallmarks of asthma, whereas Lewis rats were relatively unresponsive. Moreover, Schneider and colleagues (153) reported that there is no pulmonary inflammation and no rise in IgE after an allergen challenge in Fisher and Lewis rats.

\subsection{Neurogenic inflammation and neuronal plasticity}

Airway inflammation associated with asthma may affect neuronal activity at several points along the neural reflex pathway, including the function of the primary afferent nerves, integration within the central nervous system, synaptic transmission within autonomic ganglia and transmission at the level of the postganglionic neuroeffector junction (172). Functional changes within autonomic nerves of the lung are termed neuronal plasticity (143). As nerves regulate many pulmonary functions, altered neuronal regulation in and due to inflammation is crucial to understand. It affects the ASM tone, airway secretions (e.g. mucus hypersecretion), blood flow by vascular dilation, microvascular permeability and the migration and release of inflammatory cells (16). These effects are attributed to actions of neuropetides and tachykinins. All these insights led to the concept of neurogenic inflammation (143). The basis for this term is twofold: the biological effects produced by tachykinins in the airways in general are proinflammatory and provide a neurogenic contribution to the overall inflammatory process and the stimuli that elicit tachykinin release in the airways include mediators of inflammation (178). For instance, inflammatory mediators such as bradykinin, platelet-activating factor, $\mathrm{LTD}_{4}$, lipoxin $\mathrm{A}_{4}, \mathrm{PG}$ and histamine activate and/or sensitize capsaicin sensitive afferents (99). Hence, capsaicin desensitization reduces bronchoconstriction, vasodilation and AHR in experimental animal models (guinea pig, pig and sheep) after allergen exposure (99). In human, capsaicin led to abolished nasal irritation by lactic acid and hypertonic saline as well as absent skin flare response to allergen, pointing to the involvement of sensory nerves in allergy (99). This is supported by the finding of increased levels of neuropeptides including SP in the lungs of asthmatic patients and elevated levels of NK A in bronchoalveolar lavage fluids of asthmatic patients following airway allergen challenge (143).

The underlying mechanisms of qualitative changes in nerve functions include enhanced reflex activity, increased mediator release, enhanced sensitivity of the ASM to neuropeptides and tachykinins as well as increased receptor-density on both ASM cells and airway neurons. Moreover, quantitative changes in sensory and/or motor neurons are also debated in the context of asthma (143). LTs or TXA, for example, are inflammatory mediators enhancing acetylcholine release by activating afferent sensory nerve fibres and by directly facilitating ganglionic neurotransmission 
and acetylcholine release from vagal nerve terminals $(4 ; 7 ; 21 ; 116 ; 167 ; 176)$ (figure 5). Another mechanism leading to an increased cholinergic outflow is neuronal $M_{2}$ receptor dysfunction as acetylcholine induced feedback inhibition of acetylcholine release is prevented. Neuronal $M_{2}$ receptor dysfunction has been reported to contribute to AHR in human asthma patients, antigen challenged animals, virus infected animals, ozone exposed animals, and animals exposed to organophosphate pesticides (131). In antigen challenged animals, eosinophils are recruited to airway nerves, where they degranulate and release major basic protein (MBP), which functions as a natural occurring $M_{2}$ receptor antagonist (76) (figure 5). There is also a possible role of the inflammatory cytokine TNF- $\alpha$ to inaugurate $M_{2}$ dependent hyperreactivity, because TNF- $\alpha$ directly inhibits $M_{2}$ receptor gene expression in vitro (130). Moreover, vagally induced AHR in guinea pig after OVA sensitization (130) or viral infection (131) is abolished by etanercept (a human dimeric fusion protein consisting of the p75 TNF receptor 2 dimer linked to the fragment crystallizable $\left(F_{c}\right)$ of $\left.\lg G_{1}\right)$, and $M_{2}$ receptor expression is reconstituted by etanercept in neuroblastoma cells, which were treated with a supernatant of virus-infected tracheal epithelial cells (131).

The functional plasticity of neurons is under close control of neurotrophins (NT) (143). Trophe originates from the Greek and means nourishment. Therefore, in the context of nerves, neurotrophism describes a nutritive, target-derived factor that promotes growth and survival of neurons (142). NTs are small proteins of about $13 \mathrm{kDa}$ regulating neuronal structure and function, i.e. neurogenesis, neuronal differentiation/survival, neuronal plasticity and neuronal conduction (142). The NT family classically consists of four conserved members of similar structure and function: nerve growth factor (NGF), brain-derived neurotrophic factor (BDNF), NT3 and NT4/5. Those bind to tropomyosin-related kinase (Trk) receptors with different affinities. NGF preferentially binds to the Trk A receptor, BDNF and NT4 to the Trk B receptor, whereas NT3 binds with high affinity to the Trk $C$ receptor and lesser affinity to Trk $A$ and B. Moreover all NTs bind to the low-affinity p75 neurotrophin receptor (p75NTR) (142). Originally, NTs were reported to be involved in neurodegenerative disorders (e.g. Alzheimer's disease), brain tumors and spinal cord injury repair (142). However, there are several sources of NTs in the lung and their receptors are present at different sites. These lung components include nerves, immune cells, epithelial cells, fibroblasts, ASM, vascular smooth muscle, vascular endothelial cells and distinct immune cells (142). Accordingly, NTs have the potential to affect pulmonary function and structure at all sites. The current knowledge on NTs with respect to their importance in asthma is summarized in table 3.

Another neurotrophic factor is the glial-derived neurotrophic factor (GDNF). Primary sensory nerves are modulated by GDNF and GDNF family ligands (GFLs, e.g. neurturin, artemin, and persephin). GDNF and GLF selectively interact with the GDNF family receptors (GFR $\alpha 1-4)$ (97). Neurotrophic factor receptor expression has been largely characterized in neurons during development and in neonatal animals. In contrast, there is little information regarding neurotrophic 


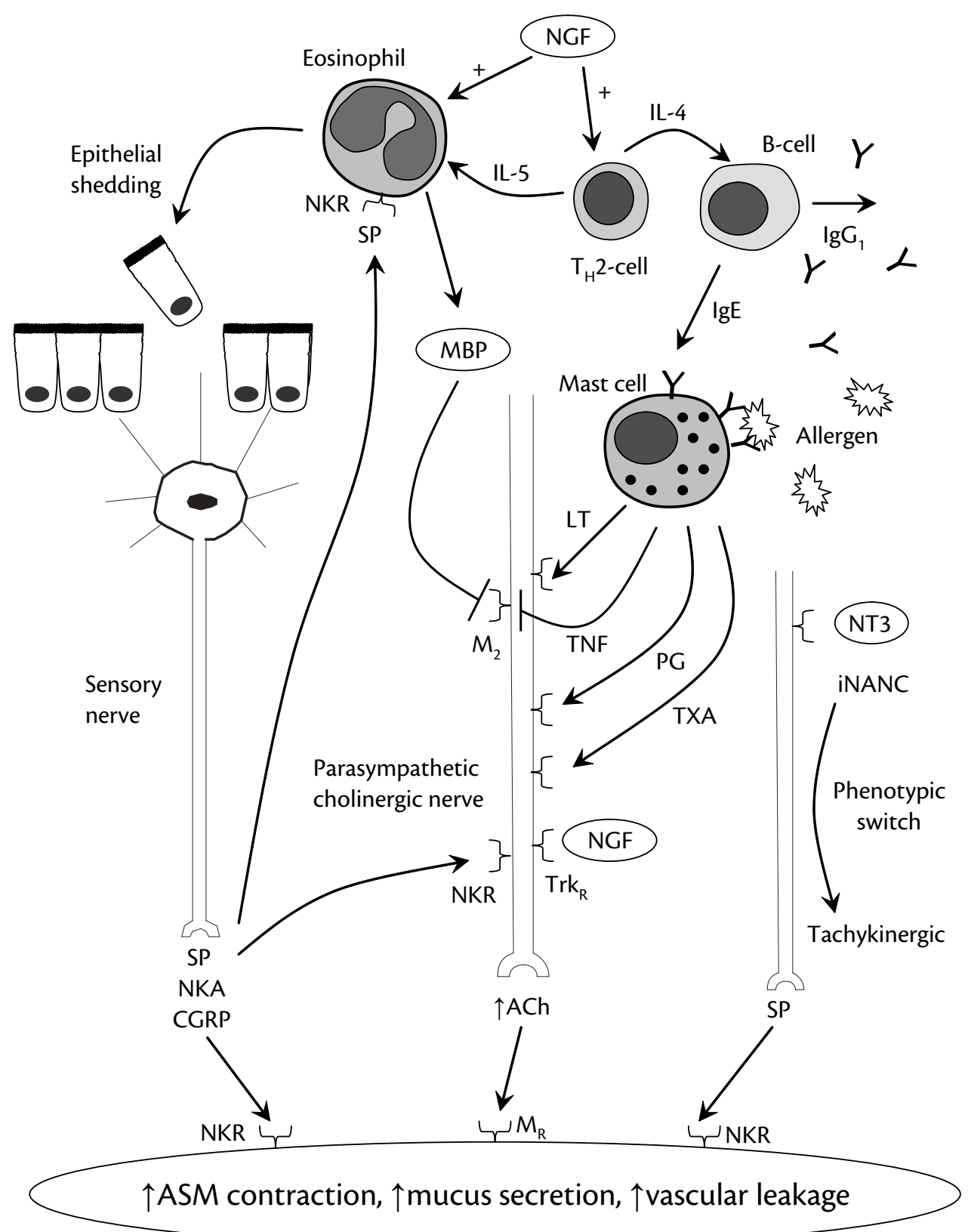

Figure 5: Irritation of nerves in inflammation and asthma. Chronic inflammation leads to epithelial shedding activating sensory nerves, which release tachykinins. Tachykinins in turn activate inflammatory cells or facilitate acetylcholine (ACh) release from parasympathetic nerves. Increased Ach outflow can also be due to muscarinic acetylcholine receptor $\left(M_{2}\right)$ dysfunction, tropomyosin related kinase receptor $\left(\operatorname{Trk}_{R}\right)$ stimulation and leukotriene (LT), prostaglandin (PG) or thromboxane (TXA) stimulation of parasympathetic nerves. $M_{2}$ dysfunction results from the natural antagonist major basic protein (MBP) released by eosinophils or from inhibited $\mathrm{M}_{2}$-gene expression by tumor necrosis factor (TNF). Neurotrophic factors may originate from different sources inducing a phenotypic switch in nerves (Neurotrophin (NT)-3 induces inhibitory nonadrenergic noncholinergic nerves (iNANC) to produce substance $\mathrm{P}(\mathrm{SP})$ ) or activating immune cells. For example, nerve growth factor (NGF) activates eosinophils and type 2 helper T-lymphocytes ( $T_{\mathrm{H}} 2$-cell). $T_{\mathrm{H}} 2$-cells release IL-5, that attracts eosinophils, or IL-4, that stimulates B-lymphocytes (B-cell) to produce immunoglobulin $(\mathrm{Ig}) \mathrm{E}$ and $\lg \mathrm{G}_{1}$. $\operatorname{gg}$ binds to the surface of mast cells, which get activated and degranulate if allergen is bound by IgE. Increased neurotransmitter (ACh, SP, neurokinin A (NKA), calcitonin gene related peptide (CGRP)) release results in increased airway smooth muscle (ASM) contraction, mucus hypersecretion and increased vascular leakage by binding to muscarinic- (Mr) or neurokinin receptors (NKR) on ASM, goblet cells, submucosal glands or vessels. $\uparrow$, increased; $\rightarrow /+$, activating, - , inhibiting. For more details, please see the text. 
Table 3: Neurotrophins and their potential function in asthma (modified from (142))

\begin{tabular}{|c|c|c|c|}
\hline Components & $\begin{array}{l}\text { NT } \\
\text { expressed }\end{array}$ & $\begin{array}{l}\text { NTR } \\
\text { expressed }\end{array}$ & Effects and importance \\
\hline \multicolumn{4}{|l|}{ Structure: } \\
\hline Nerves & $\begin{array}{l}\text { NGF; } \\
\text { BDNF; } \\
\text { NT3 }\end{array}$ & $\begin{array}{l}\text { TrkA; } \\
\text { TrkB; } \\
\text { p75NTR }\end{array}$ & 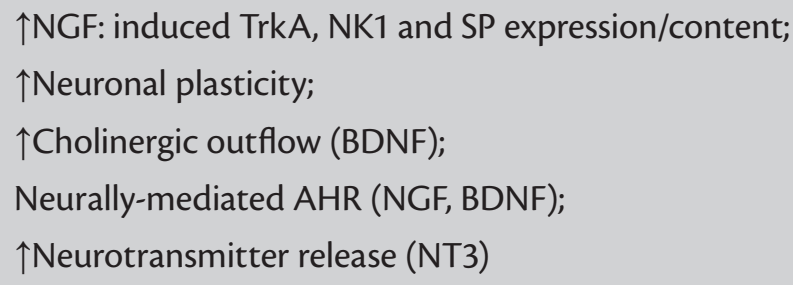 \\
\hline ASM & $\begin{array}{l}\text { NGF; } \\
\text { BDNF; } \\
\text { NT3; } \\
\text { NT4 }\end{array}$ & $\begin{array}{l}\text { TrkA; } \\
\text { TrkB; } \\
\text { TrkC; } \\
\text { p75NTR }\end{array}$ & $\begin{array}{l}\uparrow N G F, \text { BDNF, TrkA, TrkB, p75NTR with inflammation; } \\
\text { Contractility ( } \uparrow \text { with NGF, BDNF, NT4; } \downarrow \text { with NT3); } \\
\uparrow A H R \text { (NGF); } \\
\text { Airway remodelling; } \\
\uparrow C e l l \text { proliferation (NGF) }\end{array}$ \\
\hline Epithelium & $\begin{array}{l}\text { NGF; } \\
\text { BDNF; } \\
\text { NT3; } \\
\text { NT4 }\end{array}$ & $\begin{array}{l}\text { TrkA; } \\
\text { TrkB; } \\
\text { p75NTR }\end{array}$ & $\begin{array}{l}\uparrow B D N F \\
\uparrow N G F \text { with inflammation (allergic asthma); } \\
\text { Airway remodelling: } \uparrow \text { airway thickening, } \uparrow \text { cell survival }\end{array}$ \\
\hline Immune Cells: & & & \\
\hline $\begin{array}{l}\text { Monocytes/ } \\
\text { macrophages }\end{array}$ & $\begin{array}{l}\text { NGF; } \\
\text { BDNF; } \\
\text { NT3 }\end{array}$ & $\begin{array}{l}\text { TrkA; } \\
\text { TrkB }\end{array}$ & $\uparrow N G F$ and BDNF with allergen challenge \\
\hline T-lymphocytes & NGF & TrkA & $\begin{array}{l}\uparrow B D N F \text { with allergen challenge; } \\
\text { NGF produced by } \mathrm{CD}^{+} \text {and } C D 8^{+} \text {T-lymphocytes; } \\
\uparrow N G F \text { by } T_{H^{2}} 2 \text {-lymphocytes }\end{array}$ \\
\hline B-lymphocytes & $\begin{array}{l}\text { NGF; } \\
\text { BDNF; } \\
\text { NT3 }\end{array}$ & $\begin{array}{l}\text { TrkA; } \\
\text { p75NTR }\end{array}$ & $\uparrow N G F, B D N F$ and NT3 with allergen challenge \\
\hline Eosinophils & $\begin{array}{l}\text { NGF; } \\
\text { BDNF; } \\
\text { NT3; } \\
\text { NT4 }\end{array}$ & TrkA & $\uparrow N G F$ with airway inflammation \\
\hline Mast cells & $\begin{array}{l}\text { NGF; } \\
\text { BDNF; } \\
\text { NT3 }\end{array}$ & $\begin{array}{l}\text { TrkA; } \\
\text { TrkB; } \\
\text { p75NTR }\end{array}$ & Allergen-induced IgE-mediated NGF release \\
\hline
\end{tabular}

$\uparrow$, increase; $\downarrow$, decrease; AHR, airway hyperresponsiveness; ASM, airway smooth muscle; BDNF, brain-derived neurotrophic factor; $C D$, clusters of differentiation; IgE, immunoglobulin E; NGF, nerve growth factor; NT, neurotrophin; NK, neurokinin; NTR, neurotrophin receptor; P75NTR, p75 neurotrophin receptor; SP, substance P; $\mathrm{T}_{\mathrm{H}}$ 2, type $2 \mathrm{~T}$ helper lymphocyte; Trk, tropomyosin-related kinase receptor 
factor receptor expression in adult vagal afferent neurons of differing phenotypes, especially with respect to airway-specific neurons (97). Recently, Lieu and colleagues (97) showed the expression of GFR $\alpha 1-3$ and their corresponding co-signalling molecule RET in jugular neurons innervating the lung of guinea pig, but their physiological importance remains undetermined.

The mechanisms by which neurotrophic factors alter airway function are a current focus of biomedical research. Undem and coworkers (173) were the first to report a phenotypic change in airway tachykinergic innervation associated with allergen challenge and that NGF is a potential mediator for this effect. NGF increases SP expression in airway neurons and changes the neuronal phenotype so that large, capsaicin-insensitive nodose neurons with fast-conducting $A \delta$ fibers provide a component of the tachykinergic innervation (74). AHR, as shown after ozone exposure, may then result from enhanced release of acetylcholine from parasympathetic nerves, since tachykinins bind to NK receptors on these nerves, or tachykinins, i.e. SP, cause eosinophils to release MBP blocking the $M_{2}$ receptor on cholinergic nerves (179; 180) (figure 5). Recently, Pan and colleagues (138) found that the iNANC phenotype changed to a cholinergic phenotype in guinea pigs after repeated allergen challenge and that this change was mimicked by NT3 (figure 5). Physiologically, this could imply an exaggerated airway contraction, because an airway relaxant pattern is switched to a constrictive one, but the proof for that is yet missing. Furthermore, evidence is given by an electrophysiological study on airway parasympathetic ganglia from guinea pigs that synaptic transmission is directly potentiated by NGF, i.e. by binding of NGF to Trk receptors on neurons (figure 5). Using single cell recording, direct application of NGF caused a lasting decrease in the cumulative action potential after hyperpolarization and increased the amplitude of excitatory postsynaptic potentials in response to nicotinic stimulation, which was blocked by the tyrosine kinase inhibitor, K-252a, implicating Trk receptor involvement (65). NTs also exhibit profound effects on immune cells residing in airways and lung tissue, which has been termed immunological plasticity (143). In this regard, neurotrophins act as amplifiers of the locally occurring immune dysbalance. For instance, when splenic mononuclear cells from allergen-sensitized mice were stimulated with different concentrations of OVA in vitro, there was a $\mathrm{T}_{\mathrm{H}}$ 2-mediated immune response, characterized by elevated IL-4 and IL-5 production as well as increased $\lg E$ and $\lg \mathrm{G}_{1}$ synthesis (30).

The modulatory effect of nerves on inflammation or rather immune cells has been termed the inflammatory reflex (169). The modulatory effect can be either anti- or pro-inflammatory, and the role of tissue macrophages may be central to this issue. Neural anti-inflammatory regulation of tissue macrophages is local, fast and integrated through the central nervous system. Acetylcholine, originating from parasympathetic nerves, inhibits the release of cytokines from macrophages via activation of the $\alpha 7$-nicotinic acetylcholine receptor present on their surface and subsequent inhibition of gene transcription $(169 ; 171)$. This action is anti-inflammatory, because macrophages typically secrete pro-inflammatory cytokines such as TNF, IL-1, IL-6 or high-mobility group box 1 
(HMGB1) during inflammation (171). Epinephrine and norepinephrine from sympathetic nerves predominately inhibit TNF release but can, under certain circumstances, stimulate TNF release, while SP stimulates cytokine synthesis amplifying the local inflammatory response and mediating pain (169). 


\section{Aim of the study}

Irritation of nerves plays an important role in chronic inflammatory lung diseases such as asthma and COPD. Up to date, peripheral airway innervation is poorly characterized in the mammalian lung. The PCLS method is a relatively new method in pulmonary research and has been established for different species. It allows the study of large to small airways along the tracheobronchial tree and has been useful to characterize airway responses to exogenously applied mediators of the EAR and LAR. However, intrinsic activation of PCLS by stimulation of nerves has not been studied yet. Before, in vitro organ bath studies on lung innervation have been performed by EFS on tracheal strips, bronchial strips and parenchymal strips; a method that does not allow to access small airways, especially when small laboratory animals are used. Parenchymal strips do not allow to differentiate between airway and vascular smooth muscle contractions. Furthermore, studies on lung tissue in organ baths are performed under either isometric or isotonic conditions, whereas the in vivo situation is more auxotonic - as present in PCLS.

Therefore, the aim of the study was to combine the PCLS method with EFS and to establish a tool for the examination of peripheral airway innervation. It was the first aim to develop EFS of PCLS for one animal species, namely the rat, to specify EFS conditions that specifically activate nerves and exclude direct electric ASM stimulation. Neurally-evoked airway responses should be addressed pharmacologically to resolve types of innervation in peripheral airways. Since small airways respond to exogenous mediators, e.g. methacholine, differently from large airways, airways of different size should be addressed in EFS of PCLS.

After establishing the model of EFS of PCLS, lungs from allergic rats should be studieded to prove if nerves contribute to a possible AHR. Allergen and methacholine provocation of PCLS should also be investigated in the allergic rat model to evaluate a possible EAR and an unspecific AHR, respectively.

Finally, EFS of PCLS should be transferred to different species, to characterize their types of innervations, i.e. parasympathetic cholinergic, sympathetic adrenergic and/or NANC. The final aim was to compare PCLS from animals to those from humans to identify suitable animal models that allow predictions for pharmacological interventions. 


\section{Materials and Methods}

\subsection{Animals}

Harvesting of lungs from laboratory animals was approved by the local ethic committee (reference number 8.87-51.05.20.09.245). Balb/c mice (21 - $23 \mathrm{~g}$ ) were from Harlan Winkelmann (Borchen, Germany), Wistar rats (200 - 300 g) from Janvier (Le Genest St. Isle, France) or Harlan Winkelmann (Borchen, Germany), Brown Norway rats (180 - $250 \mathrm{~g}$ ) from Janvier (Le Genest St. Isle, France) and Dunkin Hartley guinea pigs (340 - $550 \mathrm{~g}$ ) from Charles River (Sulzfeld, Germany). Lungs from adult marmoset monkeys (Callithrix jacchus) were obtained from the German Primate Center (Göttingen, Germany). Sheep lungs were taken from adult Texel sheep kept at the Maastricht University Medical Center (Maastricht, Netherlands). Care and housing conditions of the animals complied with the regulations of the European Parliament and the Council Directive on the protection of animals used for scientific purposes (2010/63/EU) and the National Institutes of Health Guide for the Care and Use of Laboratory Animals.

\subsection{Human material}

Human PCLS were prepared from patients undergoing lobectomy due to cancer. After pathological inspection, cancer free lung tissue was used. The experiments were approved by the local ethics committee and all patients gave written informed consent.

\subsection{Chemicals}

Acetylcholine, aluminium hydroxide, atropine, bovine serum albumin (BSA), capsaicin, 4-diphenylacetoxy- $\mathrm{N}$-(2-chloroethyl)piperidine hydrochloride (4-DAMP), ipratropium bromide, magnesium sulphate, methacholine, neostigmine, $N_{\omega}$-nitro-L-arginine methyl ester (L-NAME), OVA grade $V(\geq 98 \%$ purity), propranolol hydrochloride, Ruthenium red, SKF96365 (1-( $\beta$-[3(4-methoxyphenyl)propoxy]-4-methoxyphenethyl)-1H-imidazole) and standard laboratory chemicals were purchased from Sigma (Steinheim, Germany). Custom Bordetella pertussis antigen was supplied by Lee laboratories (Grayson, Georgia, USA). Low-melting agarose was delivered by Gerbu (Gaiberg, Germany). Penicillin-streptomycin was obtained from Gibco (Darmstadt, Germany). [1S-[1 $\alpha, 2 \alpha(Z), 3 \alpha, 4 \alpha]]-7-[3-[[2-[($ phenylamino)carbonyl]hydrazino] methyl]-7-oxabicyclo [2.2.1]hept-2yl]-5-heptenoic acid (SQ29548) and 9,11-dideoxy-9 $\alpha, 11 \alpha$ methanoepoxy Prostaglandin $F_{2 \alpha}$ (U46619) were provided by Biomol (Hamburg, Germany). Substances for cell culture were obtained from PAA laboratories (Cölbe, Germany). 


\subsection{Cell culture medium}

The cell culture medium always comprised $2 \mathrm{mM} \mathrm{CaCl}, 1 \mathrm{mM} \mathrm{MgSO}{ }_{4}, 5 \mathrm{mM} \mathrm{KCl}, 116 \mathrm{mM}$ $\mathrm{NaCl}, 1 \mathrm{mM} \mathrm{NaH} \mathrm{PO}_{4}, 17 \mathrm{mM}$ glucose, $26 \mathrm{mM} \mathrm{NaHCO}$, $25 \mathrm{mM}$ HEPES (4-(2-hydroxyethyl)-1peperazineethanesulfonic acid), $1 \mathrm{mM}$ sodium pyruvate, $2 \mathrm{mM}$ glutamine, amino acids (PAA laboratories, M11-002, 1:50), vitamins (PAA laboratories, N11-002, 1:100) and 1\% (v/v) penicillinstreptomycin. During slicing, the medium was not supplemented by pyruvate, glutamine, amino acids, vitamins and penicillin-streptomycin.

\subsection{Preparation of PCLS}

PCLS were prepared as previously described $(188 ; 189)$ and as depicted exemplarily for the rat in Figure 6. Briefly, whole lungs of mouse, rat, guinea pig and marmoset were filled via the trachea with low-melting agarose (at $37^{\circ} \mathrm{C}$ ), whereas only separate lobes of sheep and human were filled with low-melting agarose via the main bronchus. The agarose concentration was always $1.5 \%(\mathrm{w} / \mathrm{v})$ in cell culture medium, except for mouse and rat $(0.75 \%(\mathrm{w} / \mathrm{v}))$. The filled lung tissue was cooled
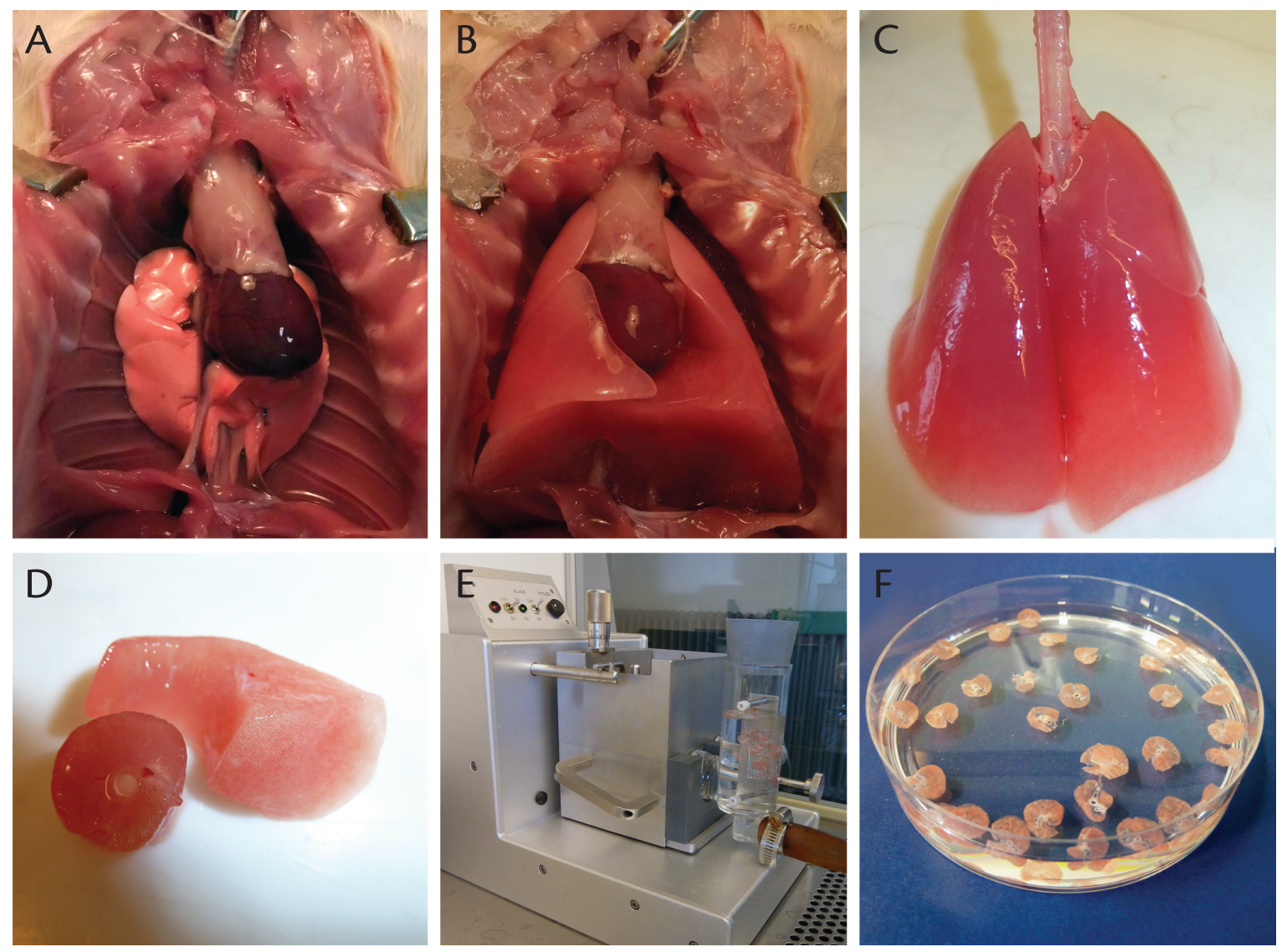

Figure 6: Preparation of precision-cut lung slices (PCLS) from rat. A. After anaesthesia the rat is tracheotomized and thoracotomy is performed so that the lung lies collapsed in the thorax. B. Low-melting agarose $(0.75 \%(\mathrm{w} / \mathrm{v}))$ is filled into the lung via the trachea and solidified on ice. $\mathbf{C}$. The lung is removed en bloc from the animal. D. The lobes of the lung are separated, cut into pieces perpendicular to the tracheobronchial tree and tissue cores containing the airway are punched. E. PCLS are cut from the cores by means of a modified self build Krumdieck tissue slicer. F. PCLS are taken in culture and are viable for at least $72 \mathrm{~h}$. 
on ice to harden the agarose. Then, tissue cores (10 $\mathrm{mm}$ in diameter) with a penetrating airway in its center were prepared by a rotating sharpened metal tube. Since the lungs from mice were too small for tissue coring, the left and right lung were separately embedded in 3\% (w/v) agarose before cutting. PCLS were cut perpendicular to the airway by means of a Krumdieck tissue slicer (Alabama Research and Development, Munford, Al, USA). PCLS were taken in culture and incubated at $37^{\circ} \mathrm{C}$ and $5 \%$ carbon dioxide atmosphere. In order to wash out the agarose and to remove cell debris as well as released mediators, the medium was changed every 30 min after PCLS preparation and every hour for the next two hours. Thereafter, the medium was changed once daily. Only slices with an airway free of agarose, beating cilia and an intact and relaxed ASM layer were used in experiments one day after preparation.

\subsection{Videomicroscopy}

The PCLS under study was placed into a cavity of a standard laboratory 12- or 24-well plate and mounted on the stage of an inverted Leica DMIL microscope (Leica Microsystems, Wetzlar, Germany). The airway area was then monitored by means of a SensiCam 365KL digital camera (Visitron Systems, Munich, Germany) at a frame rate of $0.36 \mathrm{~Hz}$ in experiments with EFS and $0.2 \mathrm{~Hz}$ in all other experiments. Camera control and image analysis were achieved by Optimas 6.5 software (Optimas, Bothell, WA, USA). The airway area before the first stimulation was defined as $100 \%$ initial airway area (IAA) (figure $9 \mathrm{~B}, \mathrm{C}$ ).

\subsection{EFS of PCLS}

EFS of PCLS were carried out in cavities of standard 12-well plates in a reaction volume of $1 \mathrm{~mL}$ cell culture medium. The PCLS (10 $\mathrm{mm}$ in diameter) were placed between two platinum electrodes of $12 \mathrm{~mm}$ distance and were weighted down by a Teflon ring $(9 \mathrm{~mm}$ outer diameter $\times 6 \mathrm{~mm}$ inner diameter $\times 4 \mathrm{~mm}$ height) (figure $9 \mathrm{~A}$ ). The electric field was applied by a HSE Stimulator II (Hugo Sachs Electronics, March Hugstetten, Germany), which allowed serial rectangular stimuli. The electric stimuli are defined by the basic EFS conditions frequency, pulse duration, current amplitude, train width and train rhythm. Their meaning is depicted in figure 7.

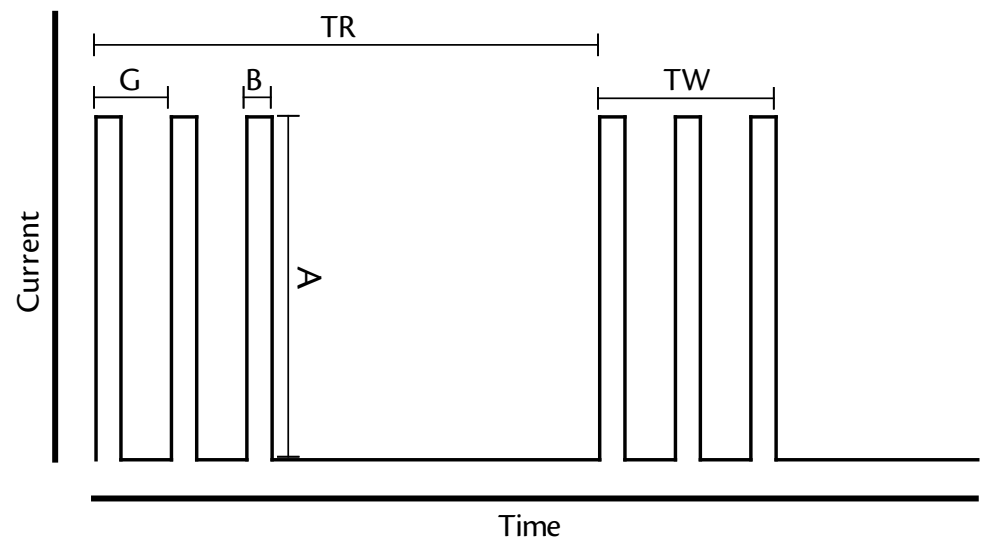

Figure 7: Basic conditions of electric field stimulation (EFS). The electric stimuli are described by the basic rhythm $(G)=1 /$ frequency $(F)$, pulse duration $(B)$, current $(A)$, train width (TW) and train rhythm (TR). 
The standard provocation protocol comprised the following setting: train rhythm TR $=60 \mathrm{~s}$, train width $T W=2.5 \mathrm{~s}$, frequency $F=50 \mathrm{~Hz}$, pulse duration $B=1 \mathrm{~ms}$ and current $\mathrm{A}=200 \mathrm{~mA}$ (equal to $40 \mathrm{~V}$ ). The standard stimulation train lasted $3.3 \mathrm{~min}$.

\subsection{Realization of response curves for the basic EFS conditions}

The basic EFS parameters frequency, pulse duration, current and train width (figure 7) were systematically examined by stimulating the PCLS repeatedly and modulating only one parameter while the others were kept constant at the values noted above (chapter 3.7). Frequency, pulse duration, current and train width were examined at $0.4-100 \mathrm{~Hz}, 0.1-5 \mathrm{~ms}, 1-200 \mathrm{~mA}$ and $0.5-20 \mathrm{~s}$, respectively. Recovery between the repetitive stimulations lasted until the airway had relaxed to its initial state ( $3-5 \mathrm{~min})$, except for frequency-response curves in species comparison where recovery lasted $1 \mathrm{~min}$.

\subsection{Examination of large and small airways in EFS of PCLS}

PCLS from rat containing differently sized airways ranging from $3.8 \times 10^{3}-1.2 \times 10^{6} \mu \mathrm{m}^{2}$ (0.07 - $1.2 \mathrm{~mm}$ in diameter) were stimulated by EFS. The first stimulation train was conducted without additive and the second train was carried out in the presence of $10 \mu \mathrm{M}$ neostigmine given $15 \mathrm{~min}$ in advance.

\subsection{Pharmacological interventions}

\subsubsection{Drugs}

Distinct drugs were used to characterize airway innervation in EFS of PCLS and exogenous activation of PCLS. The relevance of each drug for neural activation, neural specification or receptor signalling is specified in table 4.

\subsubsection{Application of magnesium}

PCLS were repeatedly stimulated by EFS. The first stimulation train took place without magnesium sulphate. After $30 \mathrm{~min}$ the second stimulation occurred either in the absence or presence of $10 \mathrm{mM}$ magnesium sulphate to block neuronal calcium entry. Magnesium sulphate was present $30 \mathrm{~min}$ prior to stimulation. After the last stimulation $10^{-4} \mathrm{M}$ acetylcholine or methacholine were added to examine airway contractility in the presence of $10 \mathrm{mM}$ magnesium sulphate. In the experiments on PCLS from rat $10 \mu \mathrm{M}$ neostigmine was present at all times. 
Table 4: Drugs and their importance

\begin{tabular}{|c|c|c|}
\hline Substance & Function & Effect \\
\hline Acetylcholine & Muscarinic receptor agonist & ASM contraction \\
\hline Atropine & Muscarinic receptor antagonist & Inhibition of ASM contraction \\
\hline Capsaicin & Vanilloid receptor agonist & Activation of sensory neurons \\
\hline 4-DAMP & Muscarinic receptor antagonist & Inhibition of ASM contraction \\
\hline Ipratropium & Muscarinic receptor antagonist & Inhibition of ASM contraction \\
\hline L-NAME & NO synthase inhibitor & Lack of NO release \\
\hline Magnesium & Competes with $\mathrm{Ca}^{2+}$ at synapse & Neuromuscular block \\
\hline Methacholine & Muscarinic receptor agonist & ASM contraction \\
\hline Neostigmine & Acetylcholine esterase inhibitor & Increased cholinergic stimulation \\
\hline Propranolol & Adrenergic $\beta$-receptor antagonist & Inhibition of ASM relaxation \\
\hline Ruthenium red & NSCC/TRP channel antagonist & Diminished eNANC response \\
\hline SKF96365 & TRP channel antagonist & Diminished eNANC response \\
\hline SQ29548 & TP receptor antagonist & Reversed U46619 agonistic effect \\
\hline U46619 & TP receptor agonist & $\begin{array}{l}\text { ASM contraction; Modulation of } \\
\text { cholinergic transmission }\end{array}$ \\
\hline
\end{tabular}

ASM, airway smooth muscle; 4-DAMP, 4-diphenylacetoxy-N-(2-chloroethyl)piperidine hydrochloride; L-NAME, $\mathrm{N}_{\omega}$-nitro-L-arginine methyl ester; NO, nitric oxide; eNANC, excitatory nonadrenergic noncholinergic nerve; NSCC, non-selective cation channel; SKF96365, 1-( $\beta$-[3-(4-methoxyphenyl)propoxy]-4-methoxyphenethyl)-1Himidazole; SQ29548, [1S-[1 $\alpha, 2 \alpha(Z), 3 \alpha, 4 \alpha]]-7-[3-[[2-[($ phenylamino)carbonyl]hydrazino]methyl]-7-oxabicyclo[2.2.1] hept-2yl]-5-heptenoic acid; TRP, transient receptor potential channel; U46619, 9,11-dideoxy-9 $\alpha, 11 \alpha$-methanoepoxy Prostaglandin $\mathrm{F}_{2 \alpha}$

\subsubsection{Characterization of EFS-induced neural airway responses}

Cholinergic response: Each PCLS from rat was exposed to three stimulation trains. The first stimulation train was carried out in the absence of any additive, the effect of acetylcholine esterase inhibition ( $10 \mu \mathrm{M}$ neostigmine) was examined in the second train and the role of acetylcholine receptors (10 $\mu \mathrm{M}$ atropine) was studied in the third train. All drugs were added 15 min prior to stimulation. In all other species than rat, the use of neostigmine as cholinergic response amplifier was not required and therefore skipped. Exceptionally in guinea pigs, PCLS were not stimulated repeatedly but separate PCLS were activated either in the presence or absence of atropine.

Sympathetic response: This response in EFS of PCLS was proven by the $\beta$-blocker propranolol. A first stimulation train was performed without propranolol as control and a second followed $15 \mathrm{~min}$ after the installation of $10 \mu \mathrm{M}$ propranolol. 
Excitatory NANC response: The unspecific TRP-channel inhibitors SKF96365 (30 $\mu \mathrm{M})$ and Ruthenium red $(10 \mu M)$ were used to validate eNANC responses in EFS of PCLS. Control stimulations were performed without these inhibitors. SKF96365 or Ruthenium red were allowed to equilibrate 15 min before the first EFS train was performed.

Inhibitory NANC response: This response in EFS of PCLS was validated by the NO synthase inihibitor L-NAME. A first stimulation train was performed without L-NAME as control and a second followed 15 min after the installation of $100 \mu \mathrm{M} \mathrm{L-NAME}$.

\subsubsection{Concentration dependent antagonism of anticholinergics}

Rat PCLS were exposed to eight consecutive stimulation trains. The first stimulation train was carried out in the absence of any additive and from the second train $10 \mu \mathrm{M}$ neostigmine was present throughout the whole experiment. In the third to eighth EFS trains the muscarinic antagonist (atropine, ipratropium or 4-DAMP) was increased cumulatively to match concentrations of $10^{-11}-10^{-6} \mathrm{M}$. All newly installed drug concentrations were equilibrated for $15 \mathrm{~min}$ prior to stimulation.

\subsubsection{Effect of the TP receptor antagonist SQ29548}

PCLS from rats were repeatedly stimulated by EFS. The first stimulation train occurred at $10 \mu \mathrm{M}$ neostigmine. The second stimulation occurred either in the absence or presence of $10 \mu \mathrm{M}$ SQ29548 to block TP receptors. SQ29548 was applied $15 \mathrm{~min}$ prior to stimulation. After the second stimulation $10 \mu \mathrm{M}$ U46619 was added to prove receptor blocking by SQ29548 and to verify a priming of acetylcholine release by $\mathrm{U} 46619$ in a following third EFS train. Airway responses during U46619 application were monitored at a frame rate of $0.1 \mathrm{~s}^{-1}$ for $15 \mathrm{~min}$.

\subsection{Exogenous activation of airway responses in PCLS}

\subsubsection{Application of methacholine}

Cumulative concentration-response curves with methacholine were performed on PCLS for various purposes. In general, methacholine was maintained at concentrations of $10^{-8}-10^{-4} \mathrm{M}$ each for $5 \mathrm{~min}$.

The effect of magnesium on ASM was studied by addition of methacholine in the presence or absence of $10 \mathrm{mM}$ magnesium sulphate. PCLS from rat were preincubated with magnesium sulphate $30 \mathrm{~min}$ before addition of the first methacholine concentration. If EFS was performed at $10 \mathrm{mM}$ magnesium, afterwards $10^{-4} \mathrm{M}$ methacholine was added in the magnesium group as in the 
control group to exclude an effect of magnesium on the ASM itself.

AHR was evaluated by provocation of PCLS from OVA sensitized or saline treated rats with methacholine $\left(10^{-8}-10^{-4} \mathrm{M}\right)$.

\subsubsection{Application of capsaicin}

Excitatory NANC nerves were triggered by application of $10 \mathrm{M}$ capsaicin on the PCLS. The airway area was monitored by videomicroscopy to quantify the response.

\subsubsection{Application of U46619}

The effect of atropine on U46619 evoked ASM contraction was evaluated. Herein, a concentrationresponse curve was conducted with $U 46619\left(10^{-8}-10^{-4} \mathrm{M}\right)$ in the presence or absence of $10 \mu \mathrm{M}$ atropine. Preincubation of atropine occurred for $15 \mathrm{~min}$ before addition of the first U46619 concentration. U46619 was cumulatively added every 5 min.

\subsection{Active sensitization of rats}

\subsubsection{Sensitization protocol}

Sensitization of male Brown-Norway rats was approved by the "Landesamt für Natur Umwelt und Verbraucherschutz Nordrhein-Westfalen" (TVA-10599G1). The sensitization scheme was in accordance to Glaab and colleagues (60) and was performed as depicted in figure 8.

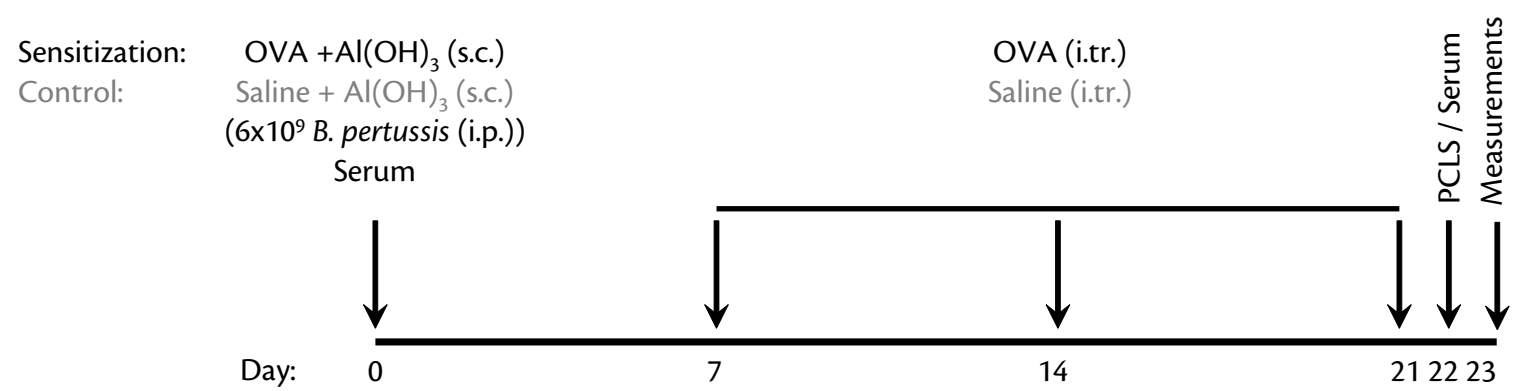

Figure 8: Acute sensitization model for rat. At day 0 animals received ovalbumin (OVA) or saline subcutaneously (s.c.). Aluminium hydroxide $\left(\mathrm{Al}(\mathrm{OH})_{3}\right)$ and heat-inactivated Bordetella pertussis (B. pertussis) organisms served as adjuvants. Sensitization was boosted at day 7, 14 and 21 by intratracheal (i.tr.) installation of OVA/saline. Precision-cut lung slices (PCLS) were prepared on day 22 and measurements were conducted the following day. Serum samples were collected at the beginning and end of the sensitization. i.p., intraperitoneal; for further please see the text.

At day 0 , rats received a subcutaneous injection of $1 \mathrm{mg}$ OVA in $250 \mu \mathrm{L}$ saline or saline only adsorbed to $20 \mathrm{mg}$ aluminium hydroxide. In some animals $6 \times 10^{9}$ heat-inactivated Bordetella 
pertussis organisms were administered intraperitoneally as adjuvants. The sensitization was boosted by intratracheal application of $100 \mu \mathrm{L} 0.3 \%(\mathrm{w} / \mathrm{v})$ OVA in saline or saline only on day 7 , 14 and 21. For intratracheal applications the animals were sedated with pentobarbital sodium $\left(50 \mathrm{mg} / \mathrm{kg}_{\text {bodyweight }}\right)$. Preparation of PCLS was performed on day 22 as described in chapter 3.5 . Serum samples were collected on day 0 and 22 for the determination of IgE titers.

\subsubsection{EFS of PCLS from sensitized animals}

EFS were performed on PCLS from OVA sensitized and control animals. EFS comprised the standard stimulation protocol (chapter 3.7) as well as the accomplishment of response curves for the frequency, pulse duration, current and train width (chapter 3.8).

\subsubsection{Allergen provocation of PCLS from sensitized animals}

PCLS from OVA sensitized or saline treated rats were provoked by $0.1 \%(\mathrm{w} / \mathrm{v})$ OVA and airway area was monitored for $20 \mathrm{~min}$ each $15 \mathrm{~s}$.

\subsubsection{Quantification IgE}

IgE levels in serum samples were determined by enzyme linked immunosorbent assay (ELISA) according to Glaab and coworkers (60). Briefly, F96 Maxisorp NUNC-Immuno Plate (NUNC, Roskilde, Denmark) were coated with a mouse monoclonal antibody against rat IgE (MCA193, AbD Serotec, Wiesbaden, Germany) in a concentration of $0.1 \mu \mathrm{g} /$ well. The plates were incubated at room temperature overnight, washed five times with $0.05 \%(\mathrm{v} / \mathrm{v})$ Tween 20 supplemented phosphate buffered saline (PBS; $137 \mathrm{mM} \mathrm{NaCl}, 2.7 \mathrm{mM} \mathrm{KCl}, 8.1 \mathrm{mM} \mathrm{Na}{ }_{2} \mathrm{HPO}_{4^{\prime}} 1.5 \mathrm{mM} \mathrm{K \textrm {H } _ { 2 }} \mathrm{PO}_{4^{\prime}}$ $\mathrm{pH}$ 7.2; sterile filtered) and blocked with $1 \%(\mathrm{w} / \mathrm{v}) \mathrm{BSA}$ in PBS for $1 \mathrm{~h}$. After aspiration of the blocking solution and washing, serum samples (diluted in PBS, 0.05\% (v/v) Tween 20, 1\% (w/v) BSA) and standard antibody (rat IgE $\kappa$, PRP07A, AbD Serotec, Wiesbaden, Germany) in the range of $15.625-1000 \mathrm{ng} / \mathrm{mL}$ were incubated for $1 \mathrm{~h}$. Following an additional washing, $100 \mu \mathrm{L}$ of peroxidase conjugated detection antibody (mouse anti rat $\kappa / \lambda$ LC:HRP, MCA1296P, AbD Serotec, Wiesbaden, Germany) at a concentration of $0.5 \mu \mathrm{g} / \mathrm{mL}$ was added per well and incubated for $1 \mathrm{~h}$ until a further washing was performed. Then, $100 \mu \mathrm{L} /$ well of 3,3',5,5'-Tetramethylbenzidine (TMB) substrate solution were applied and enzymatic substrate conversion was carried out for $15 \mathrm{~min}$ in the dark. The reaction was stopped by $50 \mu \mathrm{L} /$ well of $1 \mathrm{M}$ sulphuric acid and absorbance was measured in an ELISA plate reader (Genios Tecan, Salzburg, Austria) at $450 \mathrm{~nm}$. Wavelength correction was measured at $550 \mathrm{~nm}$ to correct for optical imperfections in the plate. 


\subsection{Statistics}

Non-linear regression, Spearman or Pearson correlation, and statistical analyses were performed using GraphPad Prism 5 (GraphPad Software, Inc., La Jolla, USA) or JMP 9 (SAS Institute, Cary, NC, USA). Homoscedasticity was checked by the Bartlett test. Unpaired data were either compared by the Mann-Whitney test or the Steel-Dwass test (if variances were unequal) or by the t-test or the Tukey test (if variances were equal). Paired data were analyzed by the paired $t$-test or linear mixed model analysis followed by the Tukey test. The statistical test used is indicated in the legends of the table or figures. The half-maximal effective frequencies $\left(\mathrm{EF}_{50}\right)$ were calculated by four parameter logistic regression and compared by the F-test. $\mathrm{P}<0.05$ was always considered significant. 


\section{Results}

\subsection{EFS of PCLS from rats - Design and Development of EFS setup}

\subsubsection{Setup and basic conditions}

Classic organ bath apparatuses were not applicable for the EFS of PCLS, because PCLS are very thin and force development due to bronchoconstriction is small, so that the use of force transducers was not practicable. In PCLS, airway responses are typically monitored by videomicroscopy (111).

A

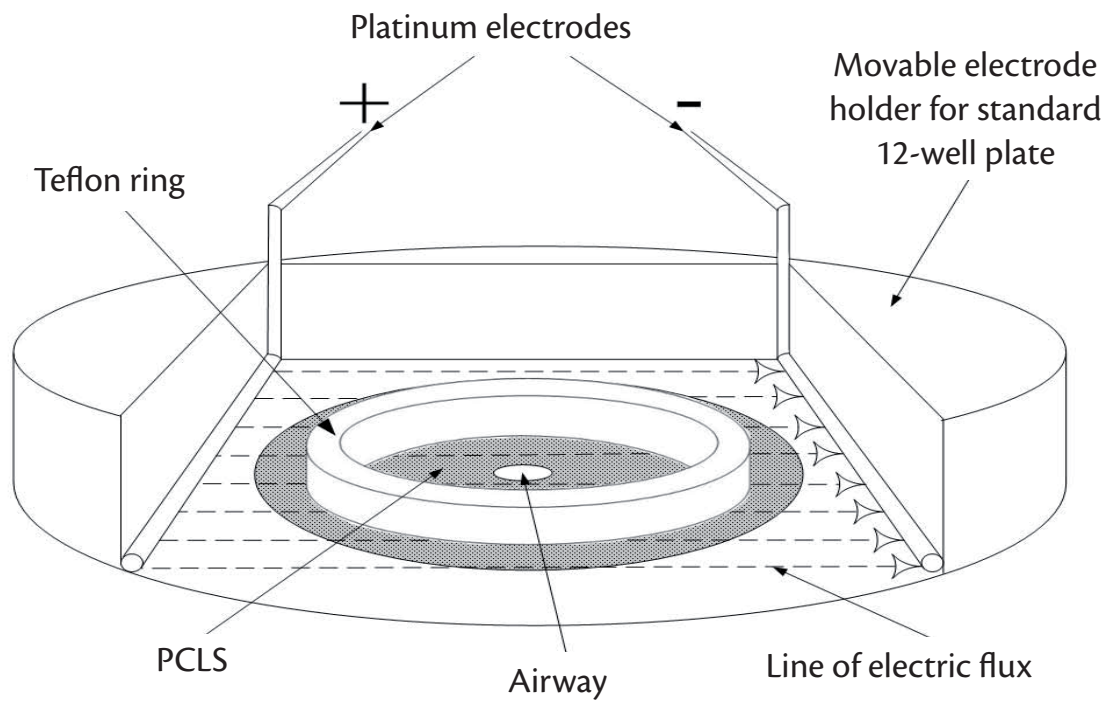

B

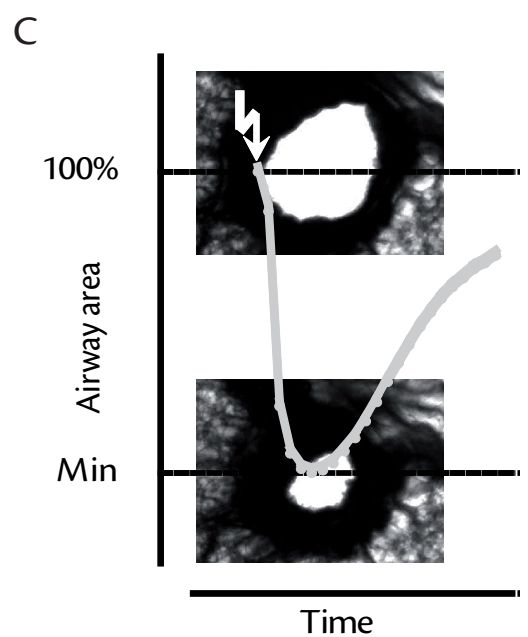

Figure 9: Setup for electric field stimulation (EFS) of precision-cut lung slices (PCLS). A. Device: PCLS of $10 \mathrm{~mm}$ in diameter were placed between two platinum electrodes, which are $12 \mathrm{~mm}$ apart. PCLS were kept in place by a Teflon ring ( $9 \mathrm{~mm}$ outer diameter $\times 6 \mathrm{~mm}$ inner diameter $\times 4 \mathrm{~mm}$ height). The electric field was applied by an electric stimulator connected to the electrodes. The electrodes were placed in a standard 12-well plate that was placed on the stage of an inverted microscope to follow bronchoconstriction by videomicroscopy. B. Photograph of a PCLS. AW, airway; PA, pulmonary artery; PV, pulmonary vein. C. Initial and minimal airway area in response to a single electric stimulation ( $F=50 \mathrm{~Hz}, B=1 \mathrm{~ms}, A=200 \mathrm{~mA}$, $\mathrm{SD}=2.5 \mathrm{~s}$, at $10 \mu \mathrm{M}$ neostigmine) is depicted. The airway area was used for quantification. 
A device was designed that allows the EFS of PCLS in a cavity of a standard 12-well plate (figure 9 A). Two platinum electrodes were held by a movable plastic carrier that fits exactly into a cavity and keeps the electrodes $12 \mathrm{~mm}$ apart. The electrodes were connected to a HSE Stimulator II allowing serial rectangular pulses. PCLS (10 mm in diameter) were placed between the electrodes, weighted down by a Teflon ring (which was figured out to be crucial to that the PCLS does not float and to establish tethering forces) and reduction in airway lumen area became apparent after electric stimulation (figures $9 \mathrm{C}$ and 10). The bronchoconstriction was reversible, as long as the Teflon ring was used (figure 10).

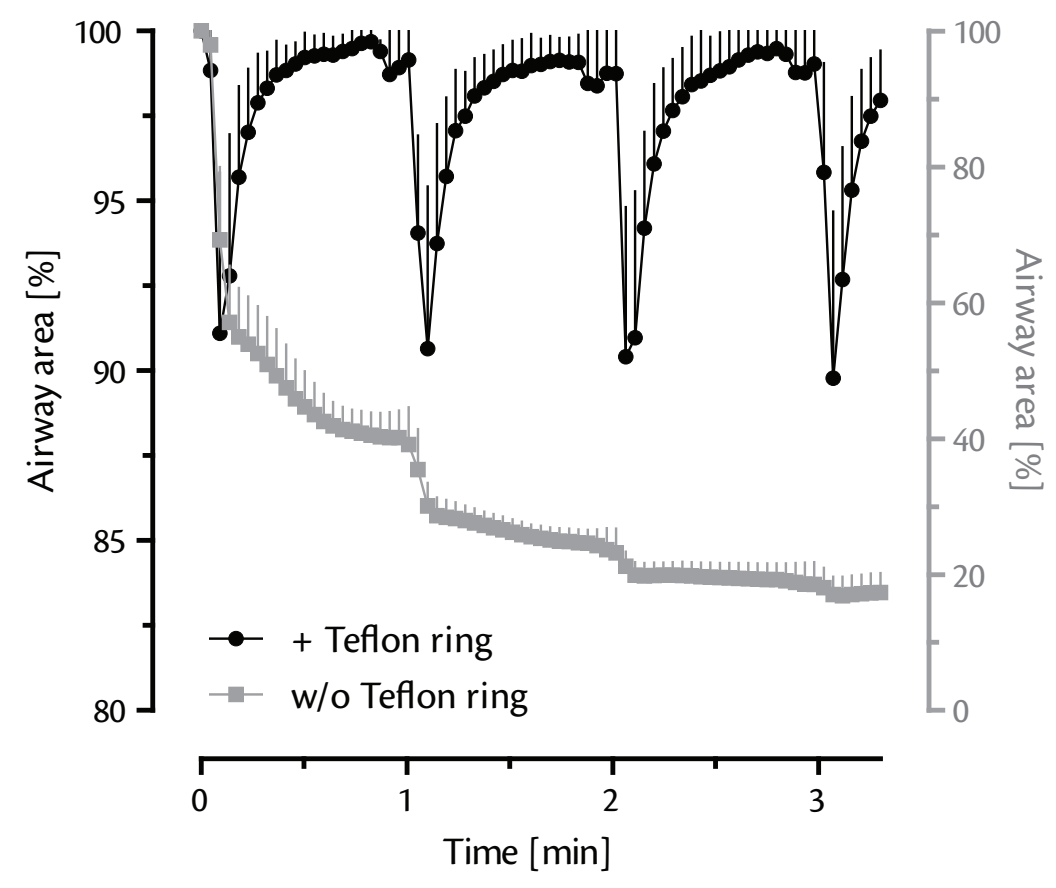

Figure 10: Role of the Teflon ring in electric field stimulation evoked bronchoconstriction in PCLS. PCLS were either loosely (right $y$-axis) placed between the electrodes or weighted down by a Teflon ring (left $y$-axis). The latter approach yielded reversible and reproducible airway responses. Data are shown as mean + SD of 6 PCLS from 2 rats in the first group or 10 PCLS from 6 rats in the group with the Teflon ring. EFS conditions were $F=50 \mathrm{~Hz}, \mathrm{~B}=1 \mathrm{~ms}, \mathrm{~A}=200 \mathrm{~mA}, \mathrm{TW}=2.5 \mathrm{~s}$ and $\mathrm{TR}=60 \mathrm{~s}$.

Since the PCLS setup differed in geometry from organ bath studies, the basic EFS conditions (frequency, pulse duration, current and train width) were evaluated initially. Airway contractions increased in a frequency-dependent manner up to $50 \mathrm{~Hz}$, (figure $11 \mathrm{~A}$ ) with a half maximal response $\left(\mathrm{EF}_{50}\right)$ at $(16.7 \pm 4.9) \mathrm{Hz}$. Bronchoconstriction also became stronger with pulse duration, current and train width. A plateau response for pulse duration was reached at pulses $\geq 1 \mathrm{~ms}$ (figure $11 \mathrm{~B}$ ). At $200 \mathrm{~mA}$ (corresponds to $40 \mathrm{~V}$ ), the maximal current output of the stimulator, airways contracted about 35\% (figure $11 \mathrm{C}$ ). Maximal ASM contraction occurred if train width lasted 10 s or longer (figure $11 \mathrm{D}$ ). However, the recovery phase to reach the initial airway area lasted longer at TW $=10 \mathrm{~s}$ (figure $11 \mathrm{D}$ ) and contractions persisted in presence of $10 \mathrm{mM}$ magnesium $((8.0 \pm 3.4) \%-$ IAA at TW $=10$ s vs. $(1.4 \pm 0.6) \%-I A A$ at TW $=2.5 \mathrm{~s} ;$ mean \pm SD; $p=0.036$ in MannWhitney test), suggesting unspecific non-neural stimulation at this train width. As a consequence 
A

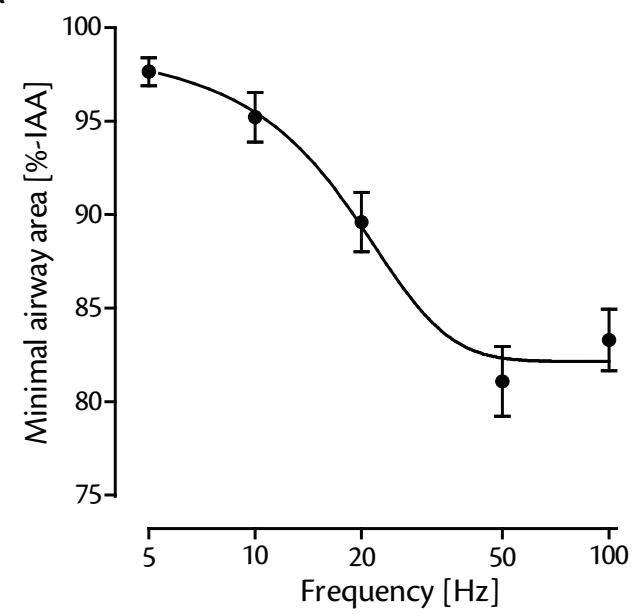

C

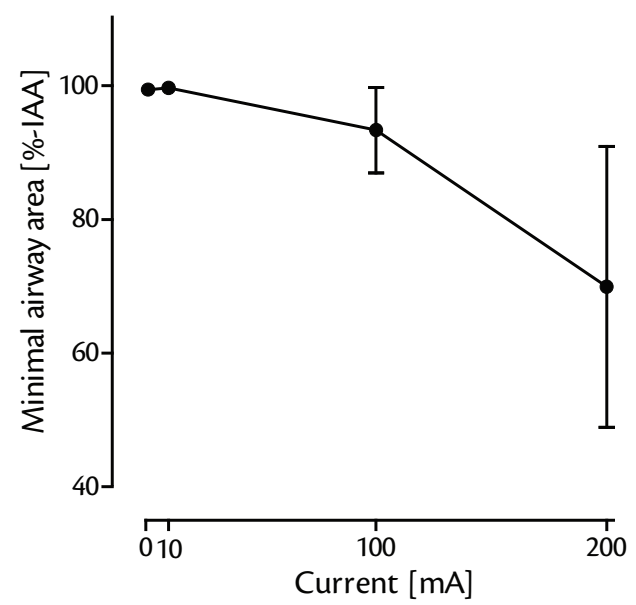

B

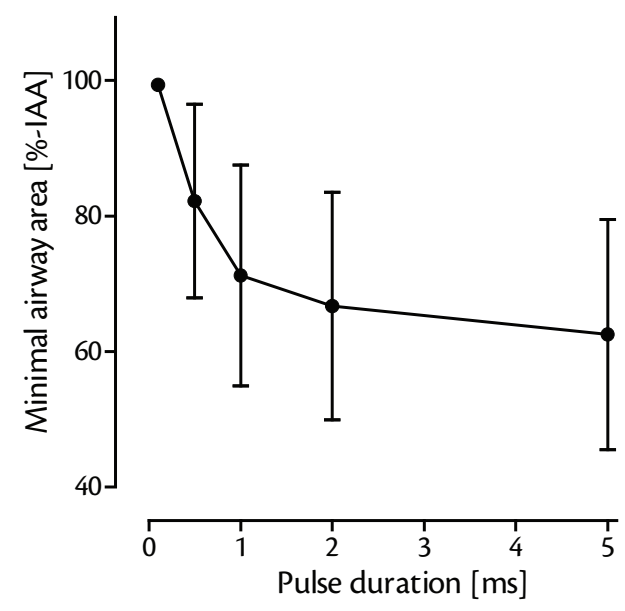

D

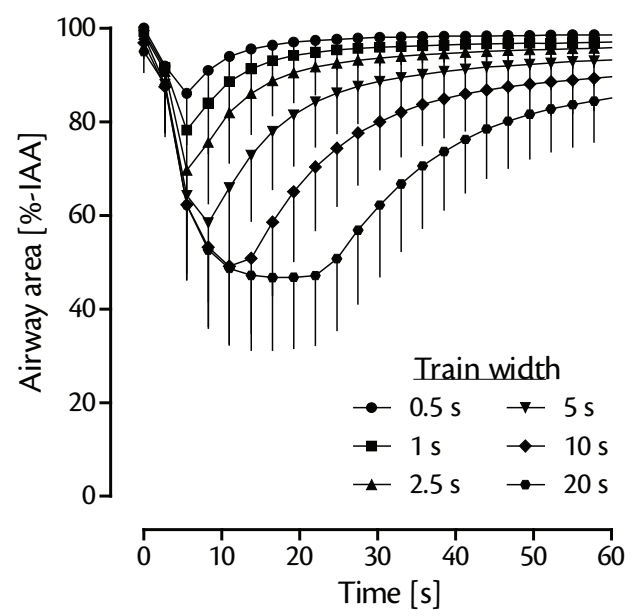

Figure 11: Determination of the basic EFS parameters to stimulate PCLS from rat. Except of the parameter that was changed in panel $A-D$, the stimulation settings were always $F=50 \mathrm{~Hz}, B=1 \mathrm{~ms}, A=200 \mathrm{~mA}$, $T W=2.5 \mathrm{~s}$. A. Frequency dependency of airway contraction by EFS. Data are shown as mean \pm SD of $11 \mathrm{PCLS}$ from 5 rats; $E_{50}=16.7 \pm 4.9 \mathrm{~Hz}$. Effect of pulse duration (B) and current (C) on airway contraction by EFS. Data are shown as mean \pm SD of 6 PCLS from 6 rats. D. Train width was increased to show maximal airway contraction in response to EFS of PCLS. Data are shown as mean \pm SD of 6 PCLS from 6 rats.

of these results, it was decided to carry out the following experiments with the Teflon ring in place, at a frequency of $F=50 \mathrm{~Hz}$, a pulse duration of $B=1 \mathrm{~ms}$, a current of $A=200 \mathrm{~mA}$ and a train width of TW $=2.5 \mathrm{~s}$. These conditions resulted in reproducible airway contractions (figure 12).

\subsubsection{Cholinergic interventions}

To examine the role of cholinergic nerves in the EFS-induced bronchoconstriction, PCLS were stimulated in the presence of neostigmine to enhance acetylcholine concentrations in the synaptic cleft and atropine to block post-synaptic acetylcholine receptors. In the presence of neostigmine $(10 \mu M)$, EFS contracted airways to $(49.2 \pm 11.0) \%$-IAA compared to $(84.1 \pm 5.3) \%-I A A$ without neostigmine (figure 12). With neostigmine still present, atropine nearly completely abolished the 
EFS-induced airway contractions (figure 12) indicating that airway contractions in rat PCLS mainly depend on muscarinic receptors.
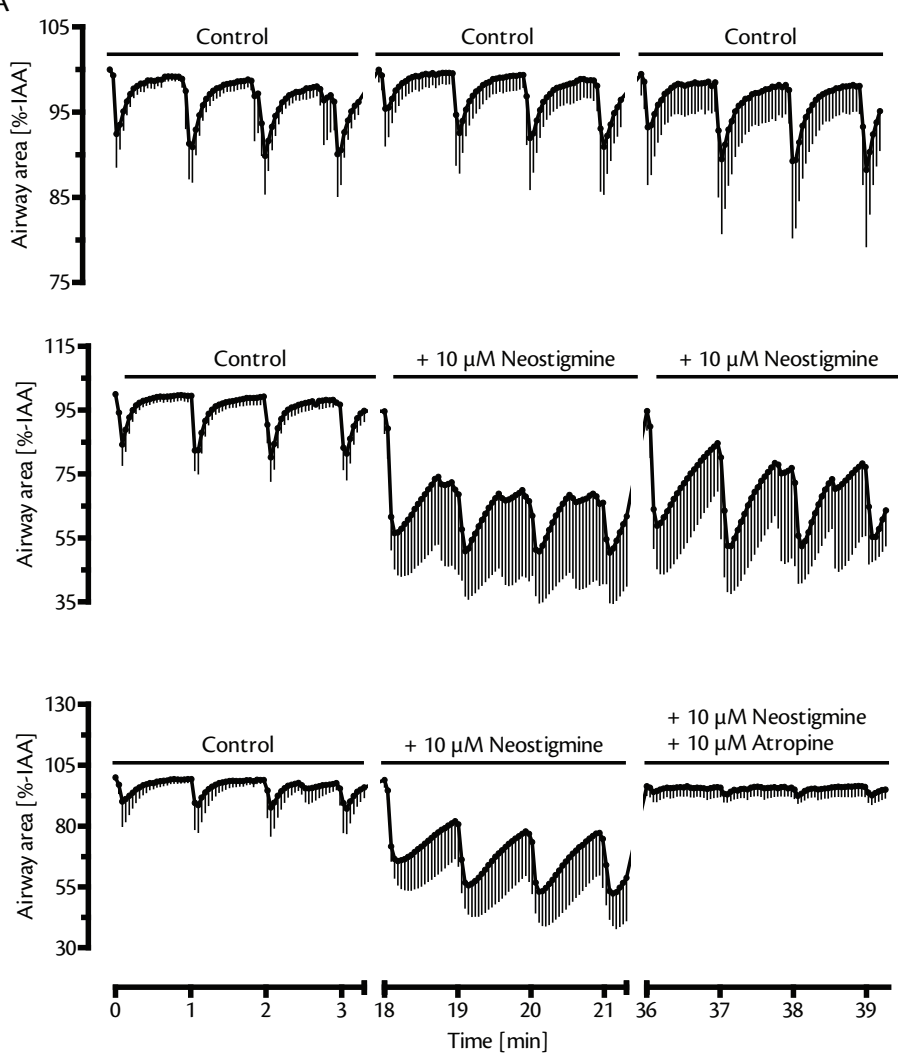
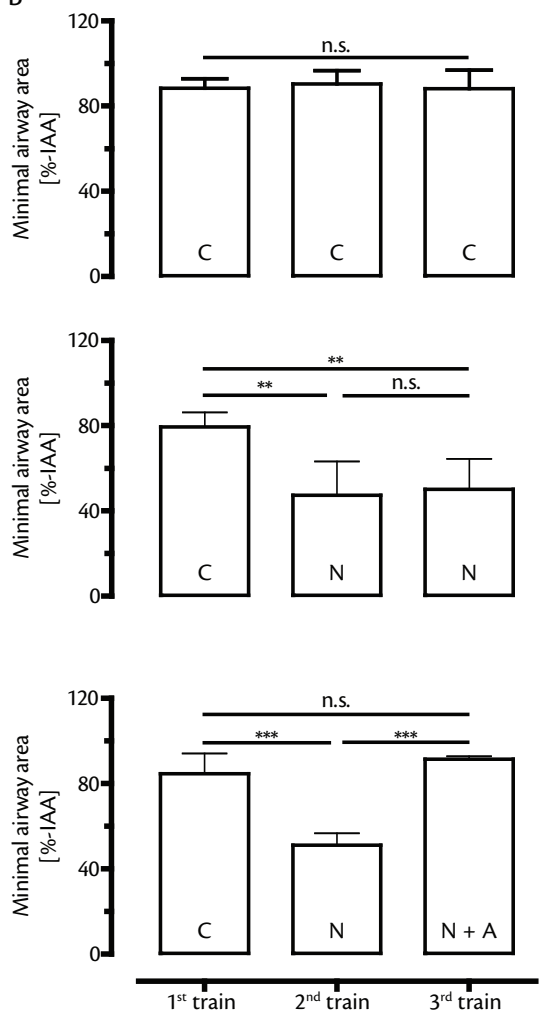

Figure 12: Cholinergic mediation of EFS-triggered airway contraction. The same PCLS was stimulated repeatedly (at $F=50 \mathrm{~Hz}, B=1 \mathrm{~ms}, A=200 \mathrm{~mA}, T W=2.5 \mathrm{~s}, T R=60 \mathrm{~s}$ ): the entire time course for a single experiment with one slice is shown on the abscissa. The time course of airway area in successive EFS trains ( 4 XEFS in $3.3 \mathrm{~min}$ ) is depicted on the left (A) and the statistical evaluation based on the minimal airway area shown as bar graphs on the right (B). The top lane shows the results of EFS without any addition to the incubation medium. The middle lane shows the results from EFS experiments in the presence of the acetylcholine esterase inhibitor neostigmine $(10 \mu \mathrm{M})$. The bottom lane shows EFS experiments in the presence of neostigmine plus the muscarinic receptor antagonist atropine $(10 \mu \mathrm{M})$. C, control EFS; N, neostigmine + EFS; $N+A$, neostigmine + atropine + EFS; n.S., not significant; ${ }^{* *}, \mathrm{p}<0.01 ; * * *, p<0.001$ in the Tukey multiple comparison test; data are shown as mean - SD (A) or mean + SD (B) from 5 independent experiments.

After validation of the cholinergic response to EFS, the setup was further developed for the testing of anticholinergic drugs. Repeatable EFS of PCLS was reversible and reproducible for at least seven consecutive stimulations after neostigmine addition, allowing successive stimulations while increasing drug concentrations at the same time (figure 13; for clarity each single spike represents the mean train of four times EFS, i.e. the total of eight stimulations mean that $28 \mathrm{EFS}$ trains occurred within in each PCLS, underlining the high reproducibility) Atropine, ipratropium and 4-DAMP showed a concentration-dependent reduction in airway contraction upon EFS. Plotting the mean minimal airway area obtained against the logarithmic antagonistic concentration revealed sigmoidal inhibition curves with significant different values of half-maximal inhibitory concentrations $\left(\mathrm{IC}_{50}\right)$. Ipratropium with a decadic logarithmic $(\log ) \mathrm{IC}_{50}$ of $(-10.0 \pm 0.4) \mathrm{M}$ was more 


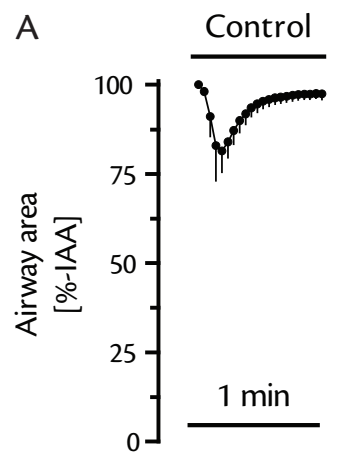

B

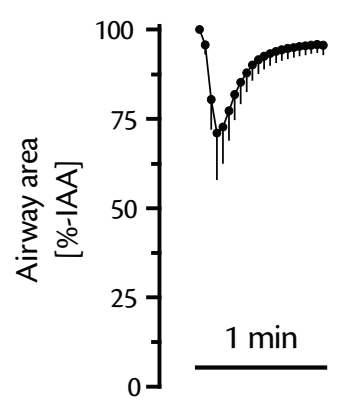

C

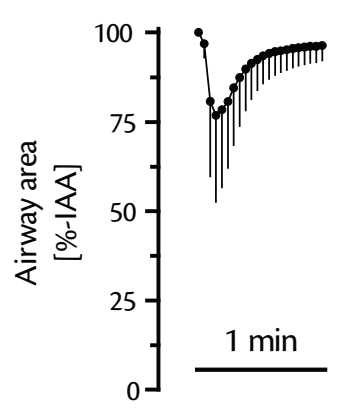

$\mathrm{D}$

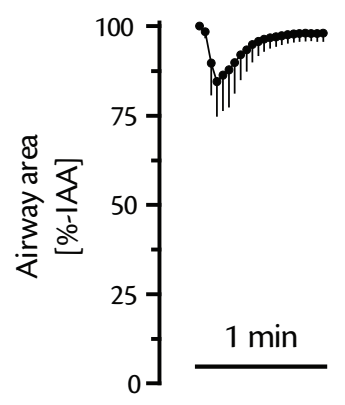

0

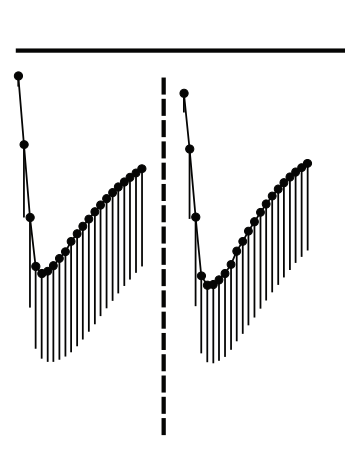

Neostigmine $\left[10^{-5} \mathrm{M}\right]$

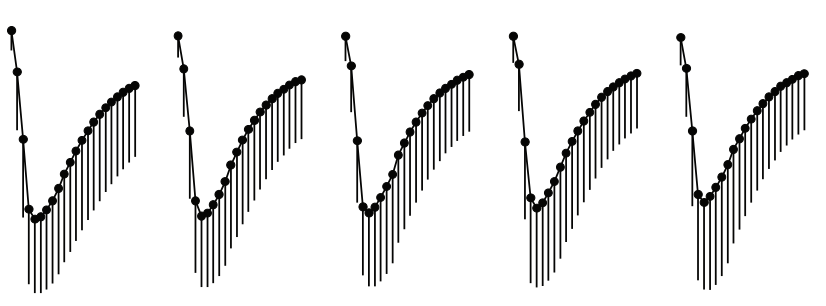

Atropine $[M]$

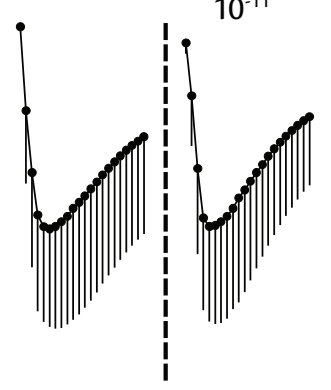

$10^{-10}$

$10^{-9}$

$10^{-8}$

$10^{-7}$

$10^{-6}$

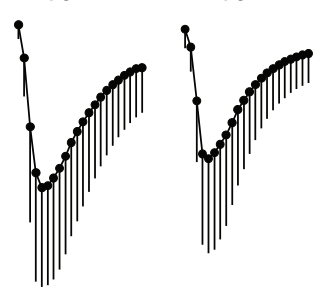

Ipratropium [M]
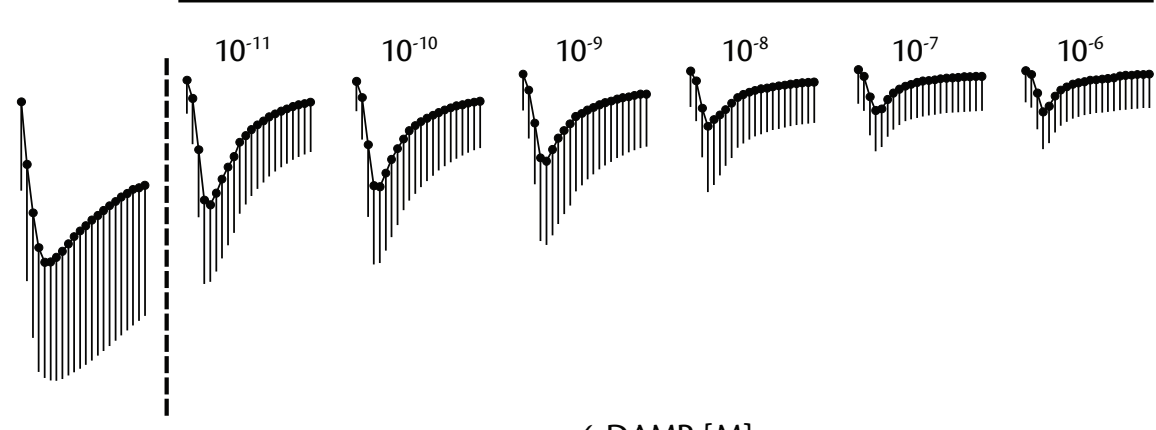

4-DAMP [M]

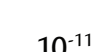

$10^{-10}$

$10^{-9}$

$10^{-8}$

$10^{-7}$

$10^{-6}$
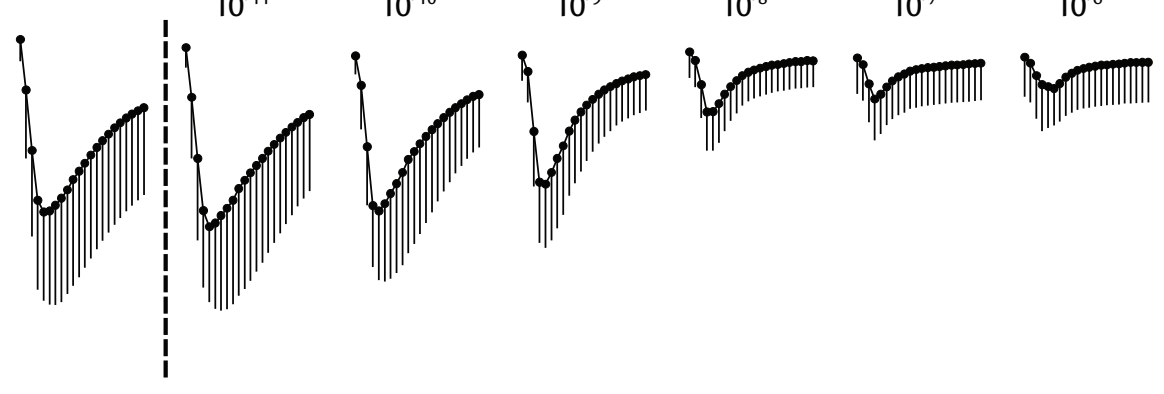

54

72

90

108

124

Point in time [min]

Figure 13: Increasing concentrations of anticholinergic drugs block airway contractions in repeated EFS of PCLS. The same PCLS was stimulated repeatedly at $F=50 \mathrm{~Hz}, B=1 \mathrm{~ms}, A=200 \mathrm{~mA}$, TW $=2.5 \mathrm{~s}$ and $T R=60$ s, i.e. a $1 \mathrm{~min}$ interval represents the mean course of $4 \times$ EFS during $3.3 \mathrm{~min}$. Drugs tested were added and equilibrated for 15 min prior to the according EFS train. Data are presented as mean - SD of 5 rats. A. Neostigmine increased airway contractions were reproducible for at least seven consecutive trains. Atropine (B), ipratropium (C) and 4-DAMP (D) were cumulatively add in consecutive EFS trains to match indicated concentrations $\left(10^{-11} \mathrm{M}-10^{-6} \mathrm{M}\right)$. In B-D like in A, neostigmine was present throughout the whole experiment beginning from the second EFS train. 


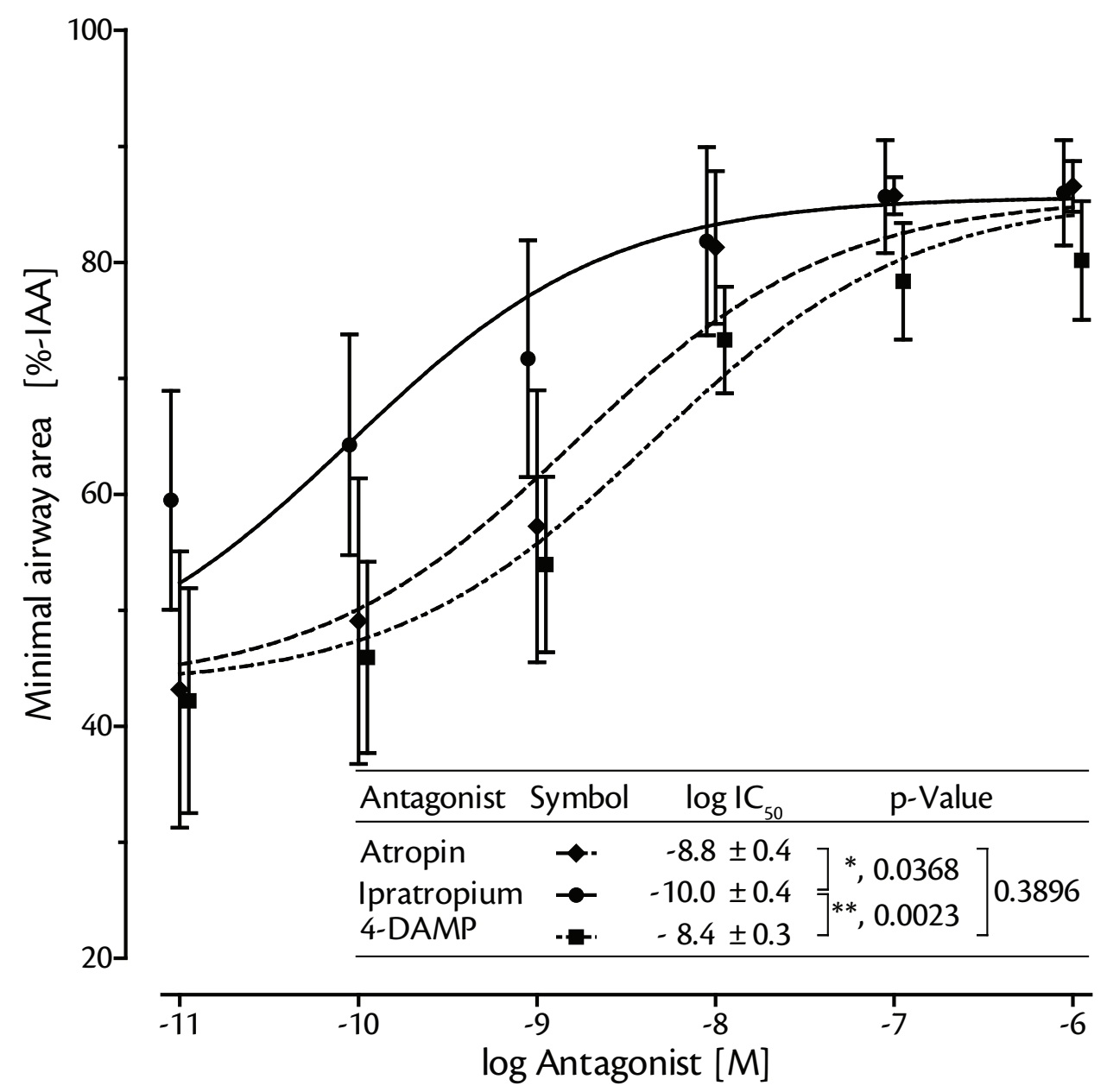

Figure 14: Ipratropium is more potent to block EFS evoked bronchoconstriction in PCLS than atropine or 4-DAMP. The mean minimal airway areas obtained in figure 13 are plotted against the decadic logarithmic (log) concentration of atropine, ipratropium or 4-DAMP. Curves were compared by performing extra sum-ofsquare F-test on top, bottom, slope and half-maximal inhibitory concentration $\left(\mathrm{IC}_{50}\right)$. Significant differences at an a-level of *, $p<0.05$ and ${ }^{* *}, p<0.01$ were found for the $\log \mathrm{IC}_{50}$ values of ipratropium and atropine or 4-DAMP, respectively; data are shown as mean \pm SEM $(n=5)$ in the graph and mean $\pm S D$ in the curve analysis table.

potent to block airway response than atropine or 4-DAMP which showed equal log $\mathrm{IC}_{50}$ values of $(-8.8 \pm 0.4) M$ and $(-8.4 \pm 0.3) M$ respectively (figure 14$)$.

\subsubsection{Effect of magnesium to prove neural activation}

The effects of magnesium concentrations high enough to block voltage-gated calcium entry in neuronal cells $(40 ; 53)$ were examined to verify that the EFS evoked airway contractions were due to neural stimulation. $10 \mathrm{mM}$ magnesium sulphate totally abolished the EFS-induced contractions in the presence of $10 \mu \mathrm{M}$ neostigmine (figure $15 \mathrm{~A}-\mathrm{C}$ ). In contrast, airway contraction by exogenously added acetylcholine was not affected by magnesium sulphate (figure $15 \mathrm{~A}-\mathrm{C}$ ) and $10 \mathrm{mM}$ magnesium sulphate did not alter the concentration-response curve of methacholineinduced bronchoconstriction (figure $15 \mathrm{D}$ ). 
A

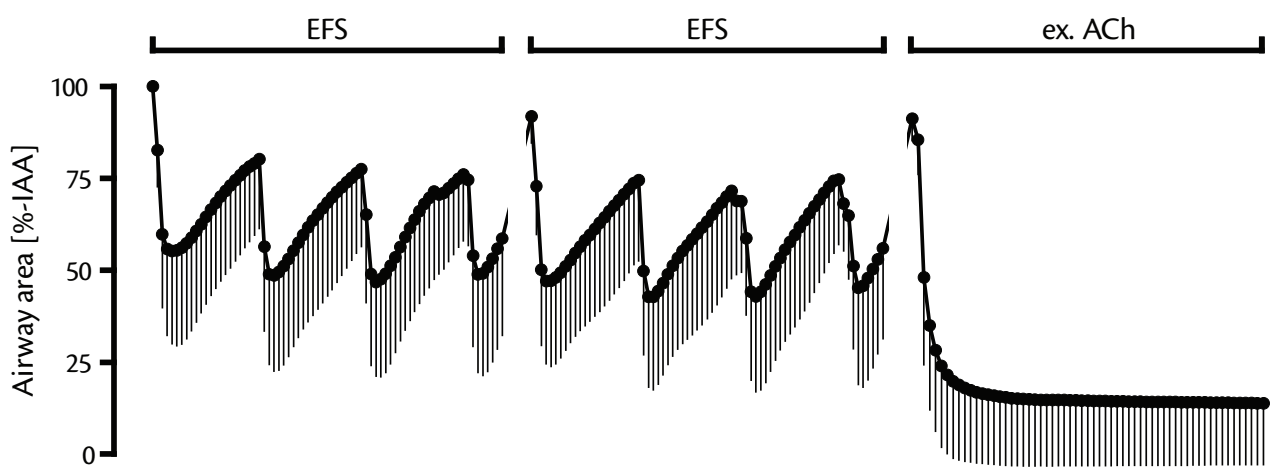

B

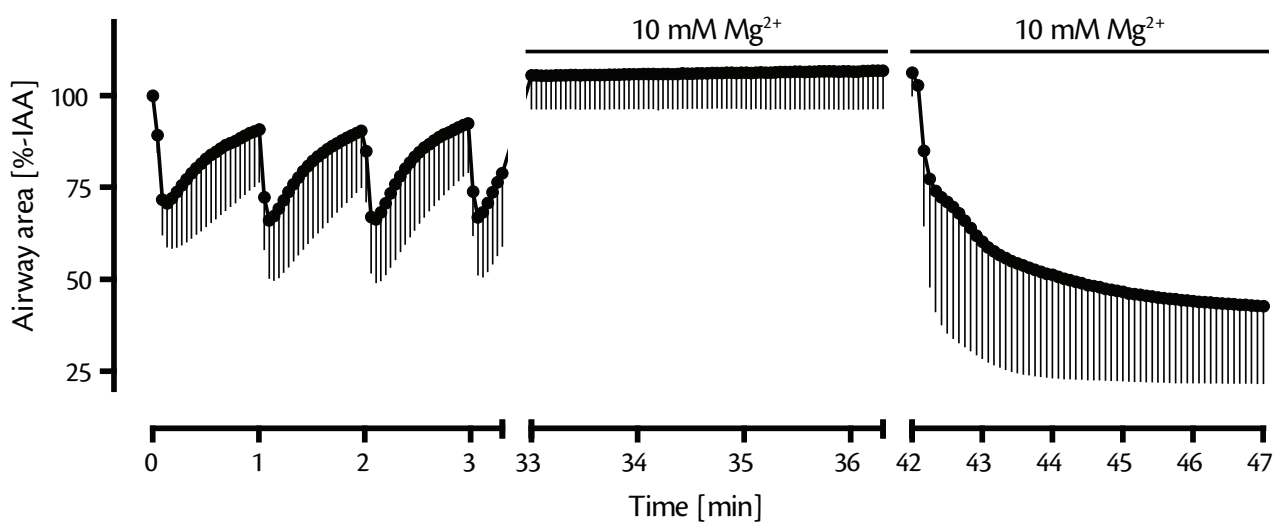

C

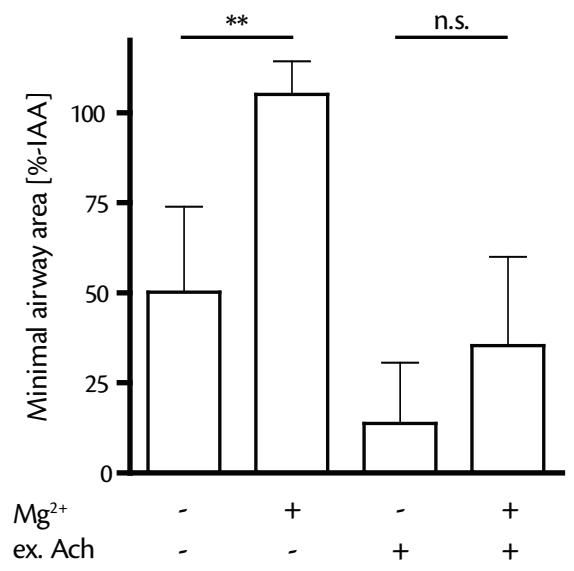

$\mathrm{D}$

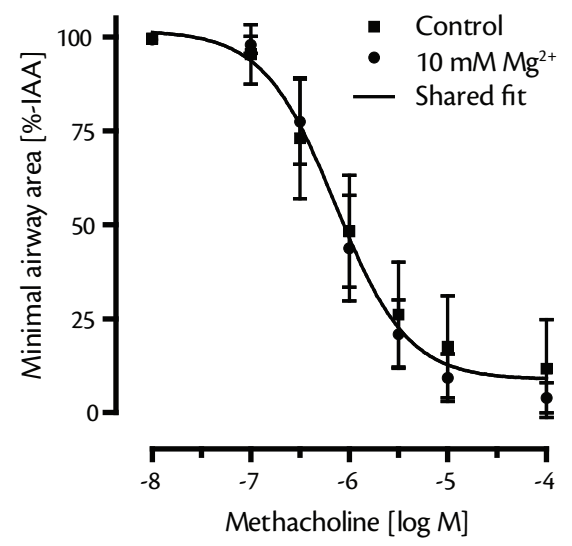

Figure 15: Effect of magnesium on airway contractions by EFS and acetylcholine derivatives. A. First and second train: $4 \times \mathrm{EFS}(\mathrm{F}=50 \mathrm{~Hz}, \mathrm{~B}=1 \mathrm{~ms}, \mathrm{~A}=200 \mathrm{~mA}, \mathrm{TW}=2.5 \mathrm{~s}, \mathrm{TR}=60 \mathrm{~s})$ in $3.3 \mathrm{~min}$ in the presence of $10 \mu \mathrm{M}$ neostigmine; third train: addition of $100 \mu \mathrm{M}$ acetylcholine; $\mathrm{n}=6$. The entire time course for a single experiment with one slice is shown on the abscissa. B. The same experiments as in panel A with the addition of $10 \mathrm{mM} \mathrm{MgSO}, 30$ min prior to the second train; $n=5$. C. Summary of the minimal airway area obtained in the experiments shown in panel $A$ and $B$ as bar chart. EFS, electric field stimulation; ex. ACh, exogenous acetylcholine; n.s., not significant in t-test of selected pair of columns; ${ }^{* *}, p<0.01$ in Mann-Whitney test of selected pair of columns; data are shown as mean - SD (A, B) or mean + SD (C). D. Cumulative concentrationresponse curves of methacholine-induced airway contraction in PCLS were recorded in the absence or presence of $10 \mathrm{mM} \mathrm{MgSO}_{4} \cdot \mathrm{MgSO}_{4}$ was added 30 min before the first addition of methacholine. Data are shown as mean $\pm \mathrm{SD}$ of $8 \mathrm{PCLS}$ for each condition from 5 animals. A shared concentration-response curve was suitable to fit the data, because there was no statistical difference at $p<0.05$ (F-test) in any parameter of the 4-parameter logistic equation between the two data sets. 


\subsubsection{Response of different airway sizes}

Since small airways respond stronger to methacholine than larger ones (111), the response of small and large airways were analyzed to EFS (figure 16).

Airway generation

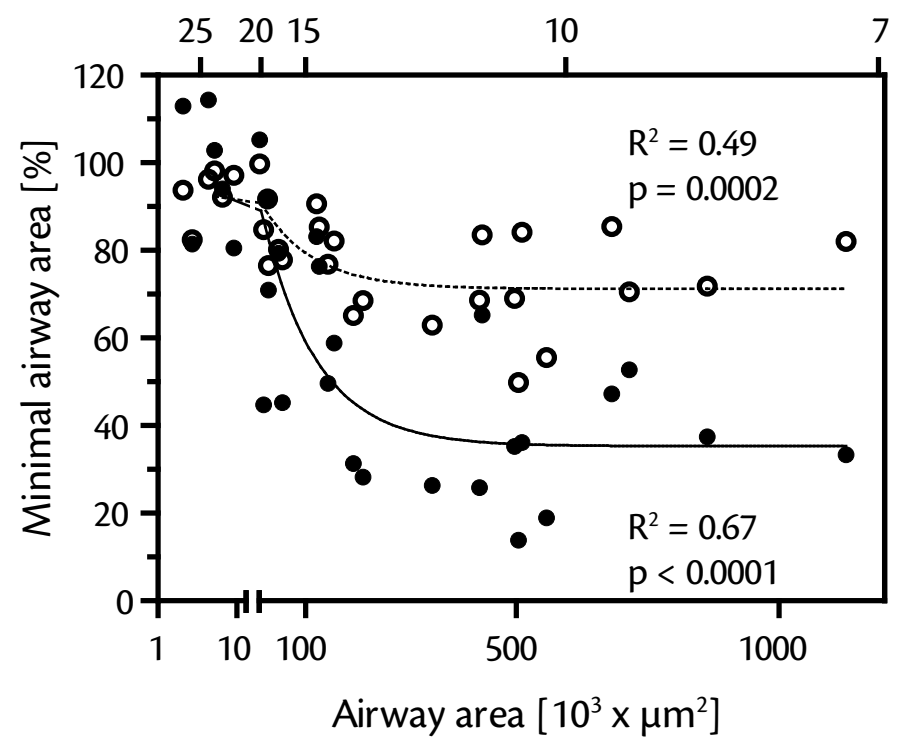

Figure 16: Correlation of airway size with EFS-triggered airway contractions. PCLS were first stimulated in the absence ( () and then in the presence $(\bullet)$ of $10 \mu \mathrm{M}$ neostigmine, i.e. the data are paired. Rat airway generations are marked on the upper $x$-axis according to Yeh and colleagues (191). A one phase exponentialdecay described the relationship reasonably well. $R^{2}$, goodness of fit; data show the results of 29 PCLS from 5 rats.

EFS were studied in PCLS with airway sizes ranging from $3.8 \times 10^{3}-1.2 \times 10^{6} \mu \mathrm{m}^{2}$ in the presence or absence of neostigmine. Herein, larger airways were more responsive than smaller ones (figure 16). Additionally, the amplification of airway contraction by neostigmine was more pronounced in larger airways.

\subsubsection{TP receptor involvement}

An interaction between TP receptor signalling and parasympathetic cholinergic acetylcholine release has been shown for dogs, guinea pigs and mice $(4 ; 7 ; 167 ; 176)$, whereas this mechanism has not yet been characterized in rat lungs. Therefore, the influence of the TP receptor antagonist SQ29548 was studied in EFS of rat PCLS. No difference was found for electrically evoked cholinergic airway responses in the presence and absence of a TP receptor antagonist (figure 17). A statistical power calculation based on these results (figure $17 \mathrm{C}$ ) indicated that at least 32 experiments were needed to possibly reach significance, indicating that any role of TP receptors would be rather small. 
A
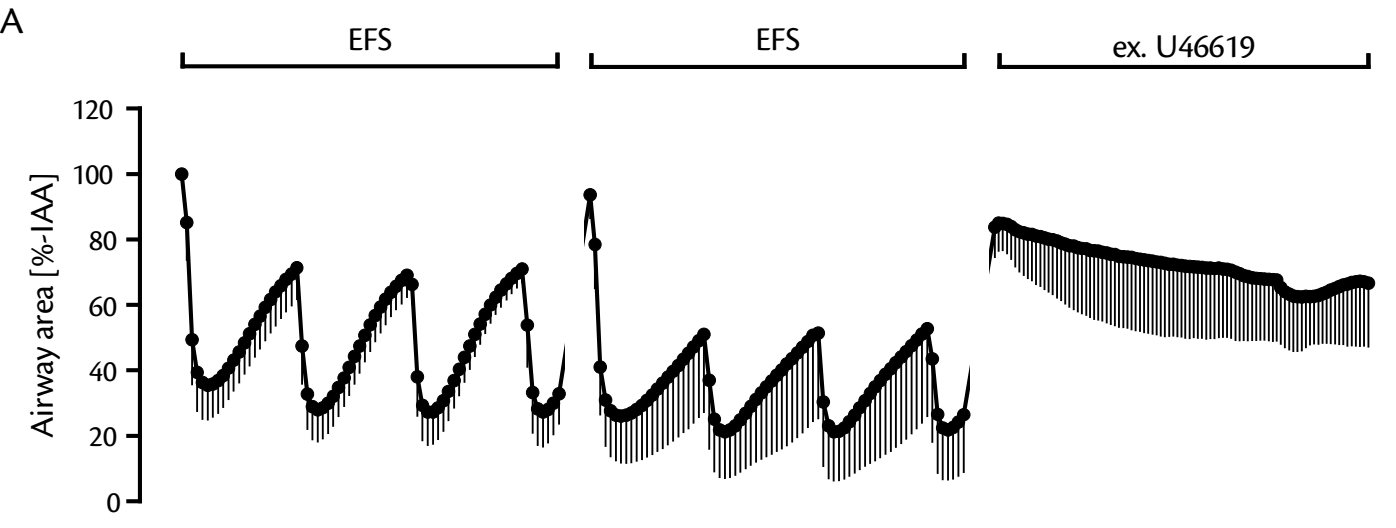

B

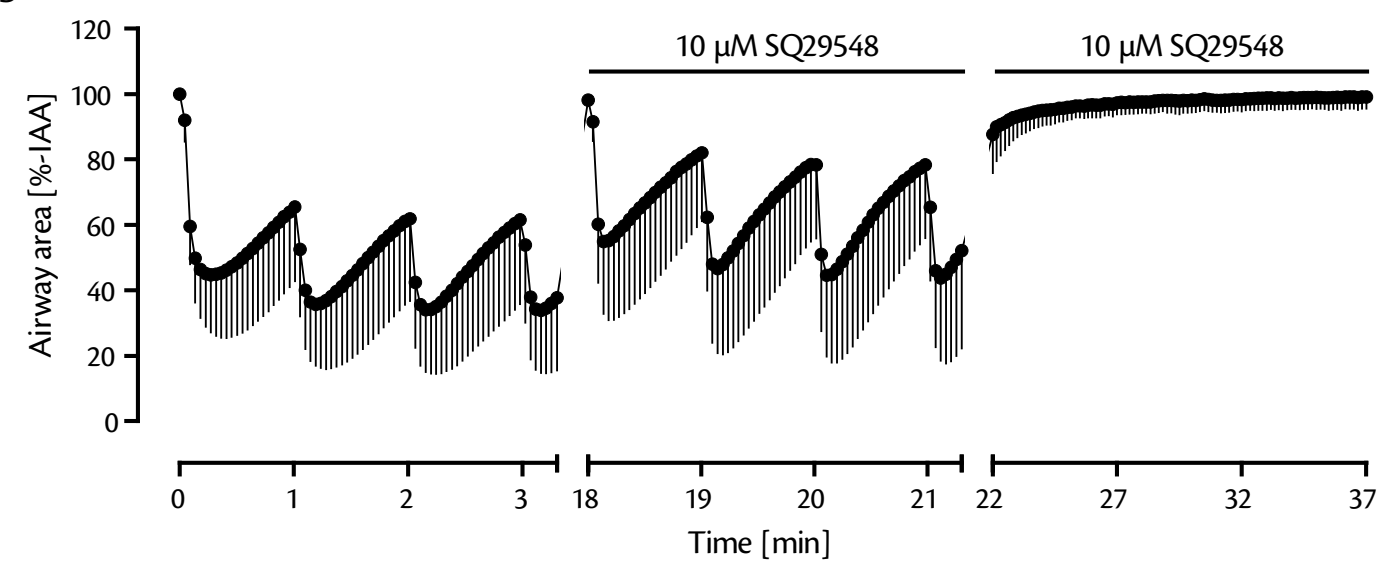

C

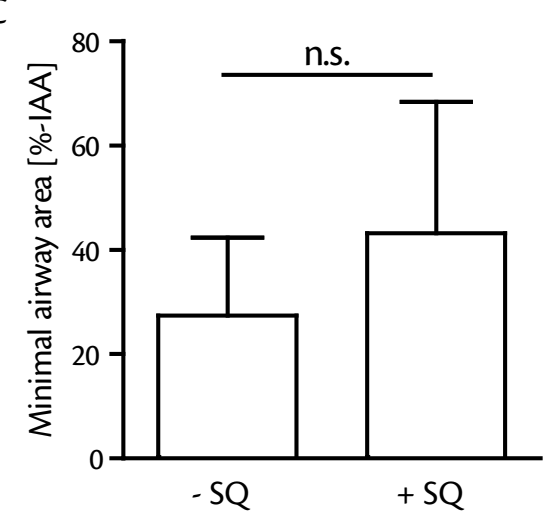

$\mathrm{D}$

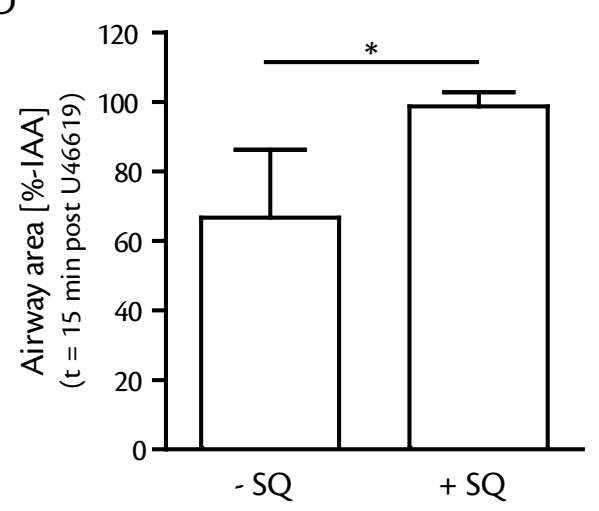

Figure 17: Effect of the thromboxane prostanoid (TP) receptor antagonist SQ29548 on bronchoconstriction induced by EFS or U46619. A. First and second train: 4 x EFS ( $F=50 \mathrm{~Hz}, B=1 \mathrm{~ms}, A=200 \mathrm{~mA}$, $\mathrm{TW}=2.5 \mathrm{~s}, \mathrm{TR}=60 \mathrm{~s})$ in $3.3 \mathrm{~min}$ in the presence of $10 \mu \mathrm{M}$ neostigmine; third train: addition of $10 \mu \mathrm{M}$ of the TP receptor agonist $U 46619 ; n=4$. The entire time course for a single experiment with one slice is shown on the abscissa. B. The same experiments as in panel A with the addition of $10 \mu \mathrm{M}$ SQ29548 15 min prior to the second train; $n=5$. C. Bar graph summary of the minimal airway area obtained in the EFS trains in panel $A$ and B; n.s., not significant in t-test. D. Bar graph summary of the airway area obtained 15 min after U46619 addition in panel $A$ and $B ;{ }^{*}, p<0.05$ in Mann-Whitney test. EFS, electric field stimulation; ex. U46619, exogenous U46619; data are shown as mean - SD (A, B) or mean + SD (C, D).

The addition of U46619 demonstrated that SQ29548 was capable of blocking TP receptors in the present system (figure 17 D). Moreover, a subsequent EFS train in the presence of U46619 did not increase the response (figure 18). 


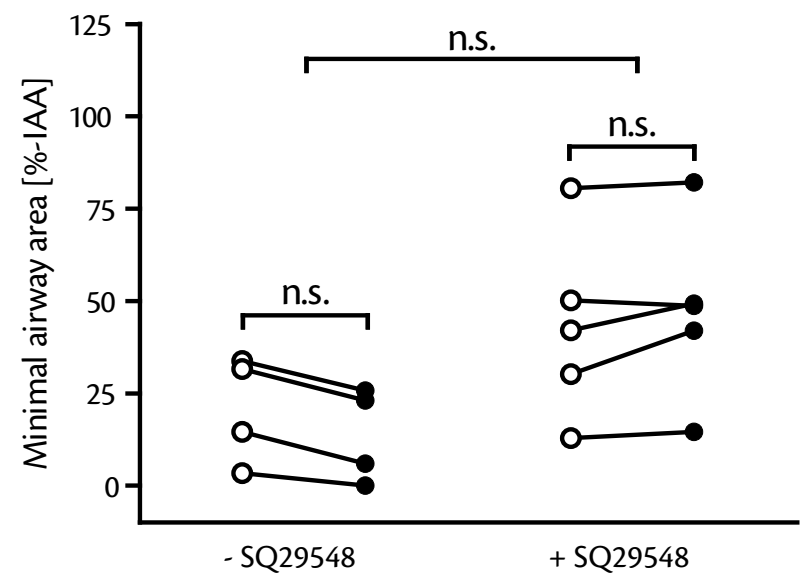

Figure 18: U46619 does not prime the airway response to EFS. A subsequent EFS train was carried out on the same set of PCLS as depicted in figure 17. For both groups ( $\pm 10 \mu \mathrm{M} \mathrm{SQ29548)}$ no significant (n.s.) difference was found before (open symbols) and after (closed symbols) addition of U46619 $(10 \mu \mathrm{M})$ in the Tukey multiple comparison test.

The influence of TP receptor agonist U46619 and its possible interactions with muscarinic receptors was studied by the use of atropine. The airway responses for cumulative concentration-response curves with U46619 were the same in the presence and absence of atropine (figure 19).

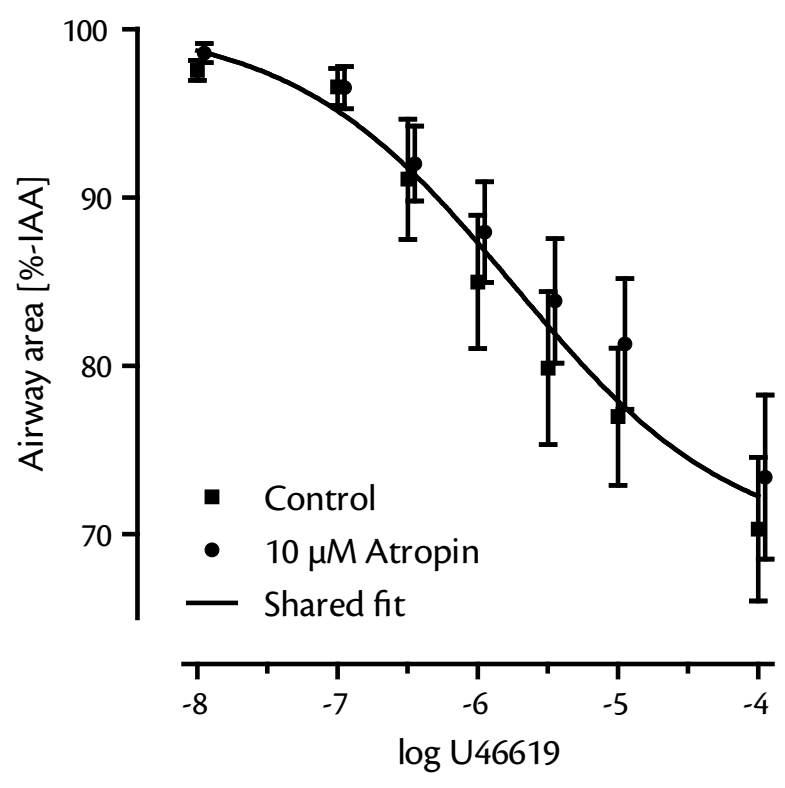

Figure 19: Effect of atropine on airway contraction by U46619. Cumulative concentration response curves of the U46619induced airway contraction in PCLS were recorded in the absence or presence of $10 \mu \mathrm{M}$ atropine. Atropine was added $15 \mathrm{~min}$ before the first addition of U46619. Data are shown as mean \pm SEM of 9 PCLS for each condition. A total of 5 animals were used for the experiment. A shared concentration-response curve was suitable to fit the data, because there was no statistical difference at $p<0.05$ (F-test) in any parameter of the 4-parameter logistic equation between the two data sets.

\subsection{Active sensitization of rats}

In the foregoing part, the EFS of PCLS was established in healthy rats. Now this method was used to study the neurally-induced bronchoconstriction of peripheral airways within an experimental rat model of asthma, since nerves may exhibit enhanced reflex activity, increased mediator release, increased receptor-density or quantitative changes during asthma (143). The ASM itself may also display enhanced sensitivity to neuropeptides and tachykinins or posses an increased receptor density (143). The sensitization occurred according to Glaab and colleagues (60), who observed an AHR in vivo and an increased number of immune cells (eosinophils, lymphocytes, neutrophils and macrophages) in brochoalveolar lavage of OVA sensitized animals. Herein, OVA adsorbed to aluminium hydroxide was injected subcutaneously, and some animals also received heatinactivated Bordetella pertussis, to initiate an intended $\mathrm{T}_{\mathrm{H}}$ 2-lymphocyte driven immune response. 
In order to boost the immune response repeated challenges with allergen were performed intatracheally assuring its deposition in the lung.

\subsubsection{Macroscopic observations}

During active sensitization, rats were either repeatedly challenged by OVA (sensitized group) or saline (control group) for three weeks. Neither control nor sensitized animals showed wheezing
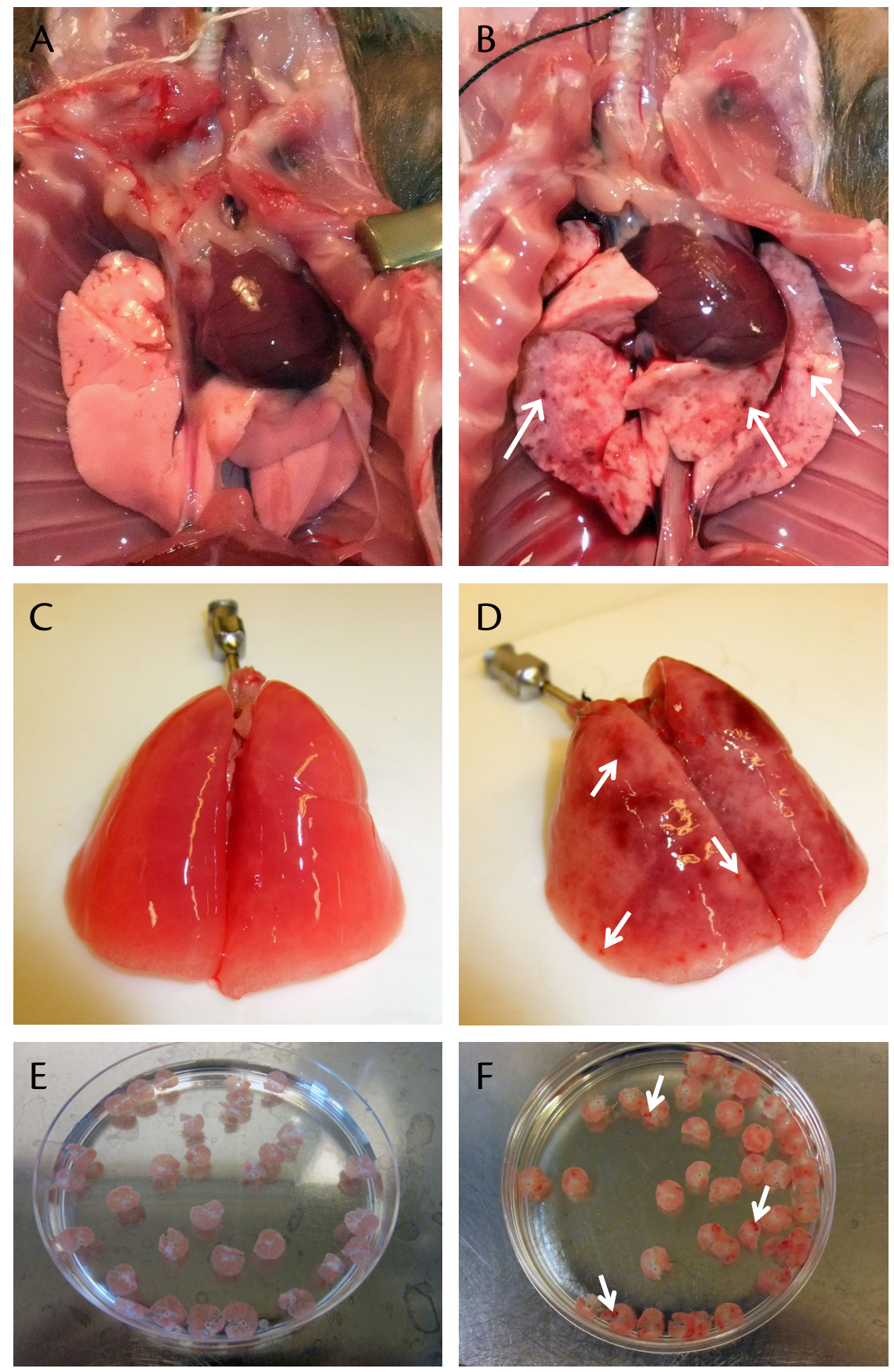

Figure 20: Ovalbumin (OVA) sensitized rats showed haemorrhages throughout the lung. Rats were actively sensitized to OVA (B, D, F) or received saline in the control group $(A, C, E)$ as described in chapter 3.12.1. A, B. The lung lied collapsed in the thorax after thoracotomy. C, D. The agarose filled lung was removed en bloc from the animal. E, F. PCLS were taken in culture. White arrows indicate haemorrhages. 
while sensitization and none of the animals appeared to be heavily sick. When rats were taken for preparation of PCLS, haemorrhages on the surface of the lung became apparent in the sensitized animals after thoracotomy, whereas in control animals haemorrhages were not present (figure $20 \mathrm{~A}-\mathrm{D}$ ). Haemorrhages in sensitized rat were spread throughout the whole lung and were still found in PCLS after the cutting process (figure $20 \mathrm{E}, \mathrm{F}$ ).

\subsubsection{Response of PCLS to allergen provocation}

To demonstrate a response to allergen after active sensitization, PCLS were provoked with $0.1 \%(w / v)$ OVA for 20 min and airway area was monitored. Only rats from OVA sensitzed rats responded with a moderate airway contraction (figure $21 \mathrm{~A}$ ).

A

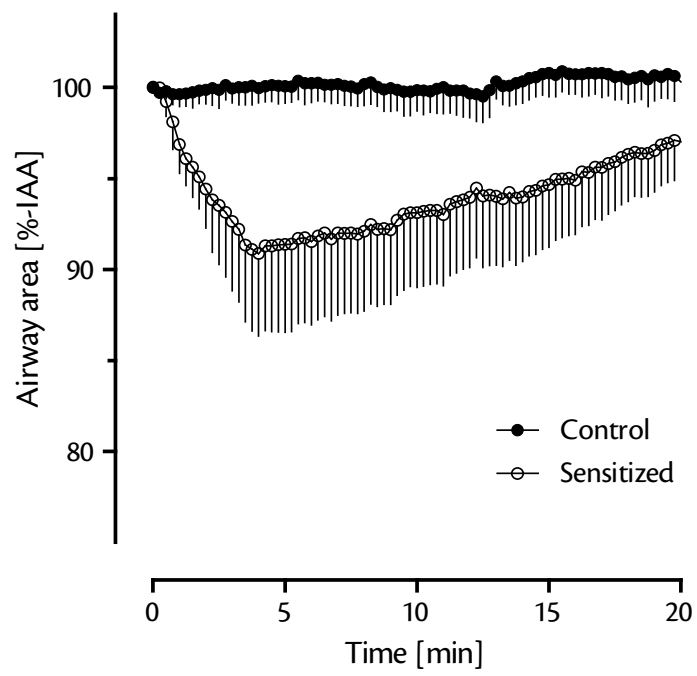

B

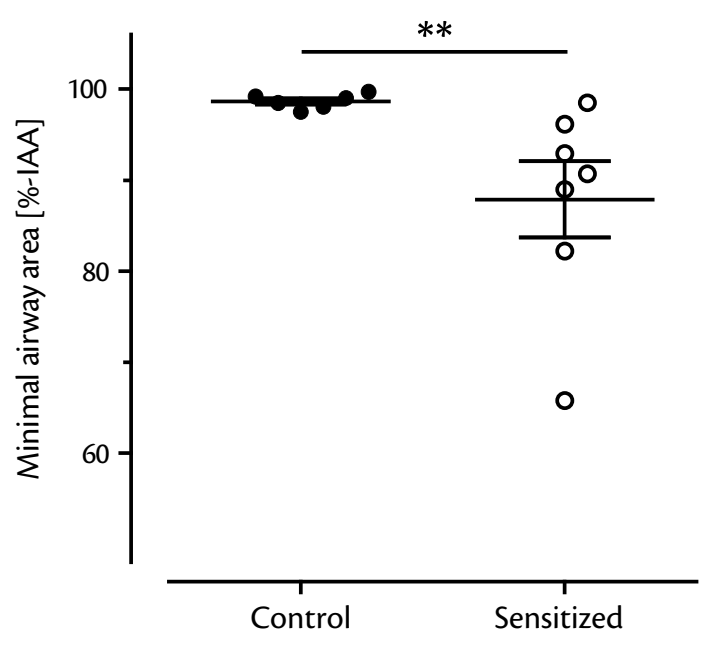

Figure 21: Allergen provocation induces bronchoconstriction in sensitzed rat. Rats were actively sensitized to ovalbumin (OVA) or received saline in the control group as described in chapter 3.12.1. Provocation of PCLS was performed by application of $0.1 \%$ (w/v) OVA for $20 \mathrm{~min}$. Groups were not further subdivided by Bordetella pertussis application, since the adjuvants did not show an effect. A. The course of airway behaviour is drawn after application of allergen. B. Maximal observed airway narrowing differed in sensitized and control group. Data are shown as mean \pm SEM $\left(n=6\right.$, control; $n=7$, sensitized); ${ }^{* *}, p<0.01$ in Mann-Whitney test.

Although a significant difference was found between the sensitized and control group, it is remarkable that some OVA sensitized rats exhibited very weak if any airway contraction at all upon OVA (figure $21 \mathrm{~B}$ ).

\subsubsection{Response of PCLS to methacholine}

Methacholine was applied to study an AHR in sensitized animals. The same concentrationresponse curve was found for both sensitized and control animals (figure 22), i.e. sensitivity (shared $\mathrm{EC}_{50}$ ) and maximal contraction (shared bottom) to methacholine were the same, and sensitized animals were not hyperresponsive. 


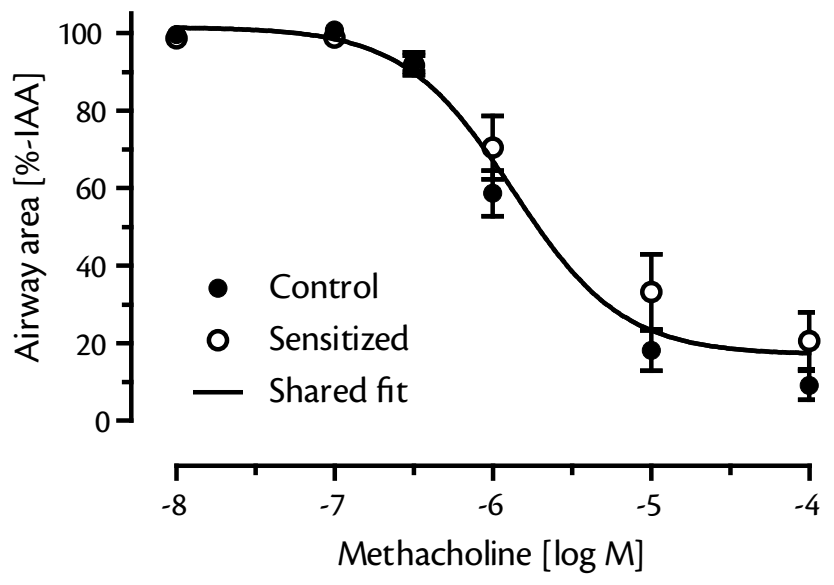

Figure 22: Sensitized animals are not hyperresponsive to methacholine. Rats were actively sensitized to ovalbumin or received saline in the control group as described in chapter 3.12.1. Cumulative concentrationresponse curves of methacholine were performed on PCLS from sensitized or control animals. Groups were not further subdivided by Bordetella pertussis application, since the adjuvants did not show an effect. Data are shown as mean \pm SEM of $n=7$ animals in sensitized group and $n=6$ in control group. A shared concentrationresponse curve was suitable to fit the data, because there was no statistical difference at $p<0.05$ in any parameter of the 4-parameter logistic equation between the two data sets.

\subsubsection{IgE levels in sensitization}

During sensitization B-lymphocytes produce specific IgE. Therefore, the IgE levels were determined to evaluate if sensitization with OVA induced the expression of IgE. Moreover, some animals received heat inactivated Bordetella pertussis organisms - a common adjuvants to trigger $\mathrm{T}_{\mathrm{H}}$ 2-cell response and in further consequence the production of IgE. In line with this, rats, which received Bordetella pertussis as adjuvants, had elevated IgE levels three weeks after sensitization in both OVA and saline treated animals (figure 23). However, OVA alone had no effect on the titers of

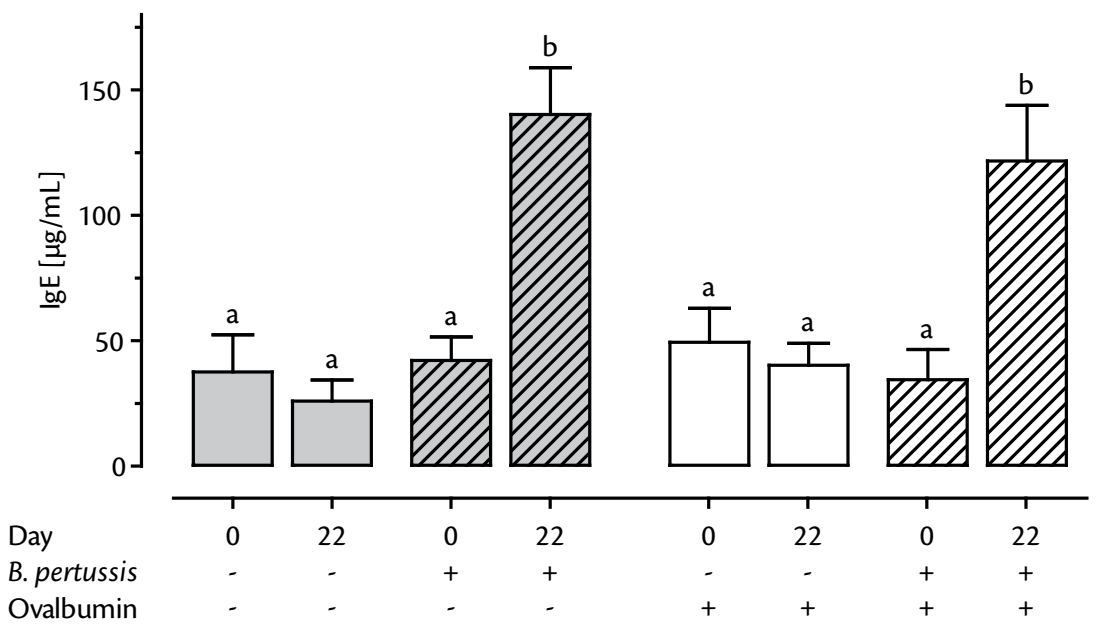

Figure 23: IgE levels after sensitization. Rats were actively sensitized to ovalbumin or received saline in the control group as described in chapter 3.12.1. Some animals also received heat inactivated Bordetella pertussis (B. pertussis) organisms as adjuvants at day 0 of sensitization. Data are shown as mean + SD. Groups not indicated by the same letter are significantly different according to the Tukey multiple comparison test. +, rats received treatment; -, rats did not receive treatment. 
IgE past sensitization (figure 23). Notably, the anti-lgE antibody used in the underlying ELISA only detected total IgE but not anti-OVA specific IgE.

\subsubsection{Response of PCLS to EFS}

Frequency, pulse duration, current and train width are the basic parameters by which the electric field can be determined. Nerves are differentially activated by changes in the electric field and airway responses are altered. Bronchoconstriction occurred with an increase in each parameter. A shared frequency-response curve with an $\mathrm{EF}_{50}$ of $(18.9 \pm 3.2) \mathrm{Hz}$ was found for OVA sensitized and saline control animals (figure $24 \mathrm{~A}$ ). Furthermore, no significant differences were observed in response to increasing pulse durations or train widths for both groups (figure $24 \mathrm{~B}, \mathrm{D}$ ). However, the sensitized animals seemed to be less reactive. This was most prominent for the sensitivity to increasing currents (figure $24 \mathrm{C}$ ).

A

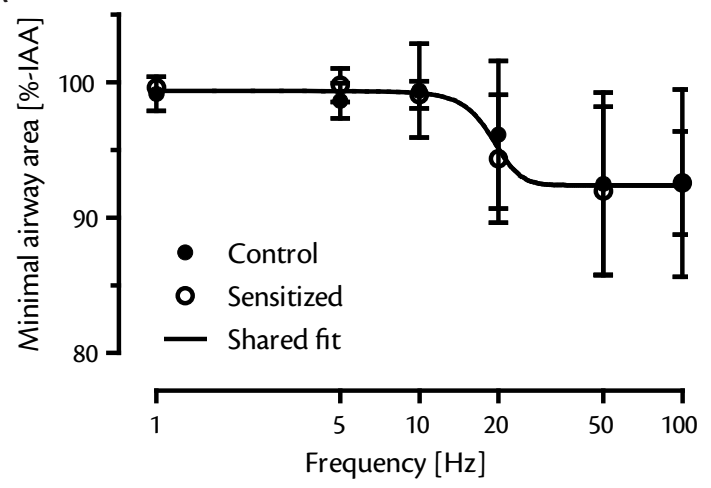

C

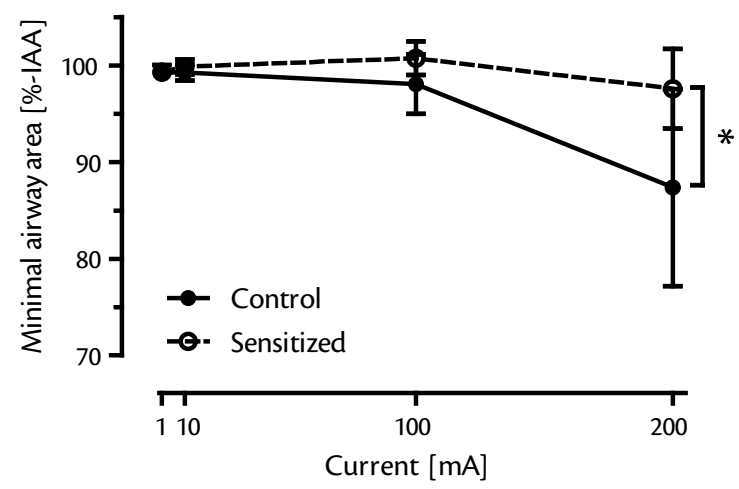

B

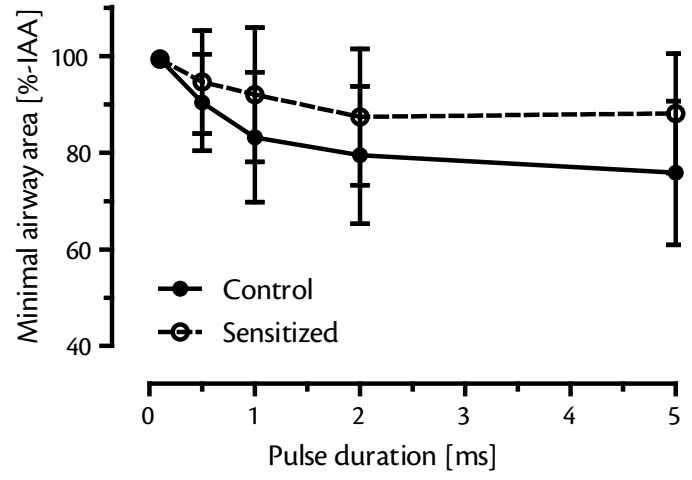

D

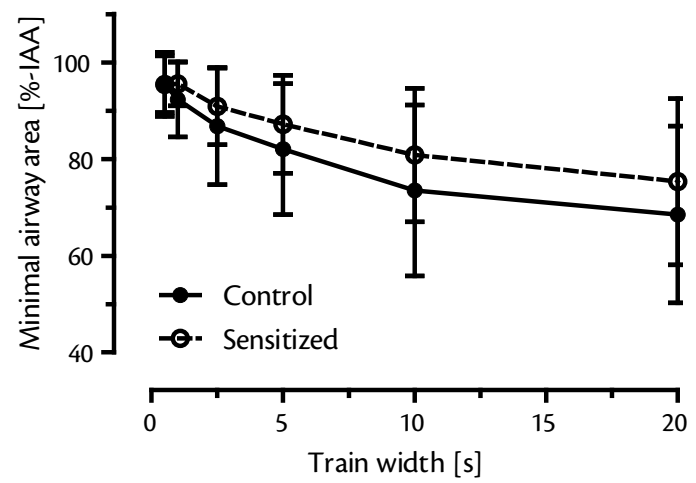

Figure 24: Response of PCLS from sensitized and non-sensitized rats to basic EFS parameters. Rats were actively sensitized to ovalbumin or received saline in the control group as described in chapter 3.12.1. Except of the parameter that was changed in panel $A-D$, the stimulation settings were always $F=50 \mathrm{~Hz}$, $B=1 \mathrm{~ms}, A=200 \mathrm{~mA}$, TW $=2.5 \mathrm{~s}$. A. The frequency-response curve resulted in a shared curve with an $\mathrm{EF}_{50}$ of $(18.9 \pm 3.2) \mathrm{Hz}$. B, C and $\mathbf{D}$ show the effect of pulse duration, current and train width, respectively, on airway contraction in EFS of PCLS from actively sensitized or control animals. Data are allways shown as mean \pm SD ( $n=7$, sensitized group, $n=6$ control group); ${ }^{*}, p<0.05$ in unpaired $t$-test on the area under the curve.

Deriving from the study of the basic parameters above, one can conclude, that the standard stimulation parameters $(F=50 \mathrm{~Hz}, \mathrm{~B}=1 \mathrm{~ms}, \mathrm{~A}=200 \mathrm{~mA}, \mathrm{TW}=2.5 \mathrm{~s})$ as determined during the 
development of EFS (chapter 4.1.1) are also appropriate here when examining sensitized and nonsensitized rats. Therefore, the responses of PCLS from either sensitized or saline treated rats were exposed to the standard stimulation conditions. Interestingly, a significant difference was figured out in which PCLS from the OVA treated rats were less reactive (figure 25), even though the small difference may not be physiologically relevant.

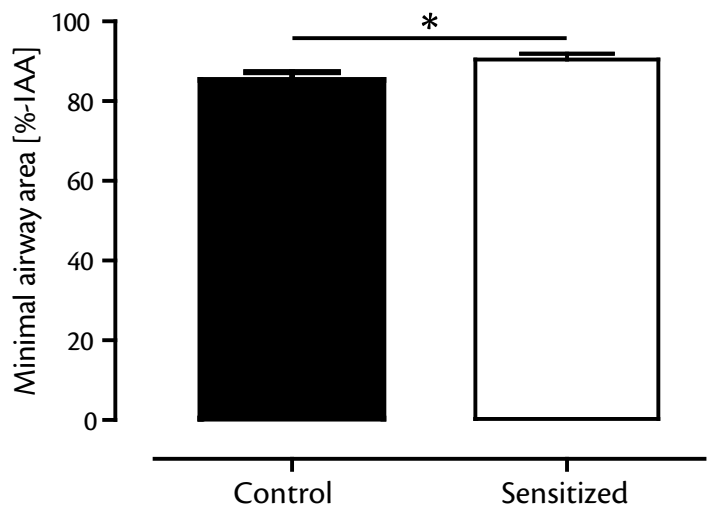

Figure 25: Response of PCLS from sensitized and non-sensitized rats to standard EFS conditions. Rats were actively sensitized to ovalbumin or received saline in the control group as described in chapter 3.12.1. EFS was carried out at $F=50 \mathrm{~Hz}$, $B=1 \mathrm{~ms}, \quad A=200 \mathrm{~mA}$ and $T W=2.5 \mathrm{~s}$. Data are shown as mean + SEM of 47 PCLS from 7 sensitized rats and $43 \mathrm{PCLS}$ from 6 saline control rats; *, $p<0.05$ in unpaired t-test.

Since there was the probability that not all rats were effectively sensitized the airway response to standard EFS of PCLS from both control and OVA treated group was plotted against their response to allergen provocation. However, Spearman analysis did not result in any correlation (figure 26).

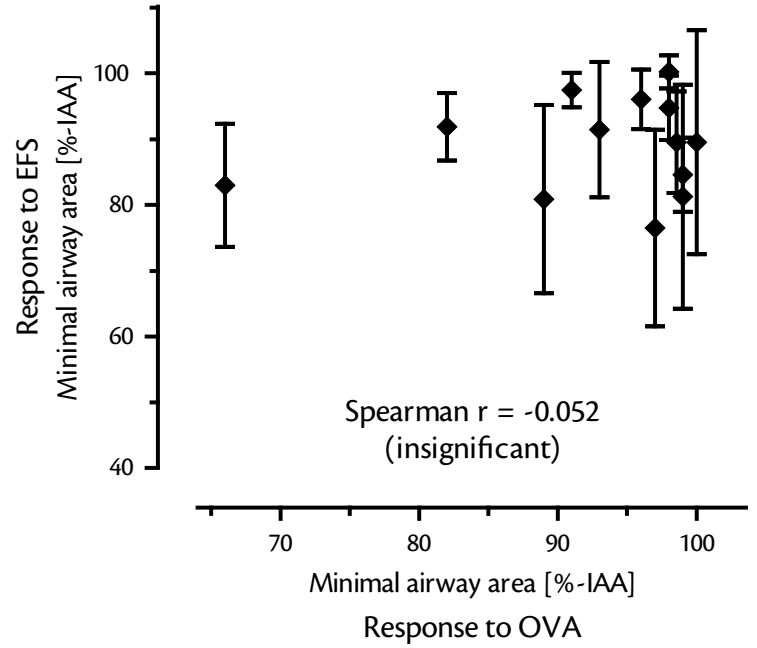

Figure 26: There is no correlation between airway responses to EFS and response to ovalbumin (OVA). The mean $\pm \mathrm{SD}$ response to standard EFS ( $F=50 \mathrm{~Hz}, B=1 \mathrm{~ms}, \mathrm{~A}=200 \mathrm{~mA}$ and $T W=2.5 \mathrm{~s}$ ) of each rat out of the sensitization experiment (chapter 3.12.1), i.e. sensitized and control rats are combined and each data point reflects a single animal, is depicted against the according response to OVA provocation (figure 21). Spearman correlation resulted in $r=-0.052$ pointing to an insignificant correlation.

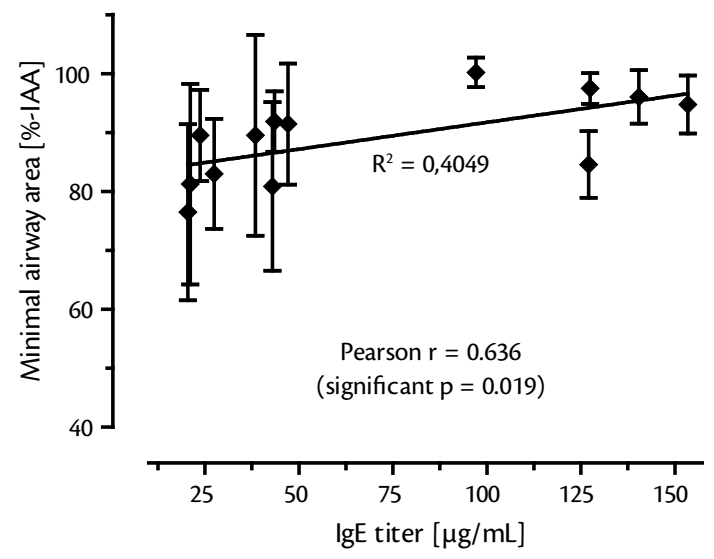

Figure 27: Airway response to EFS is correlated to the IgE titer. The mean $\pm S D$ response to standard EFS $(F=50 \mathrm{~Hz}, B=1 \mathrm{~ms}, A=200 \mathrm{~mA}$ and TW $=2.5 \mathrm{~s}$ ) of each rat out of the sensitization experiment (chapter 3.12.1), i.e. sensitized and control rats are combined and each data point reflects a single animal, is depicted against the IgE level obtained after 3 weeks of sensitization (figure 23). Pearson correlation resulted in significant correlation $(r=0.636, p=0.019)$, and consequently a linear regression was conducted. 
Moreover, as the IgE level is indicative of the immune status of the animal, the airway response to EFS of PCLS from both control and OVA treated rats was correlated to the IgE levels (figure 27). Pearson analysis resulted in a significant dependence $(r=0.636, p=0.019)$. As Pearson correlation assumes a linear dependence, a linear regression was performed showing lesser reactivity with higher IgE titer (figure 27).

\subsection{Species comparison}

\subsubsection{Responses and sensitivity to EFS}

PCLS from the various species responded differently to EFS; please note the different scales in

A
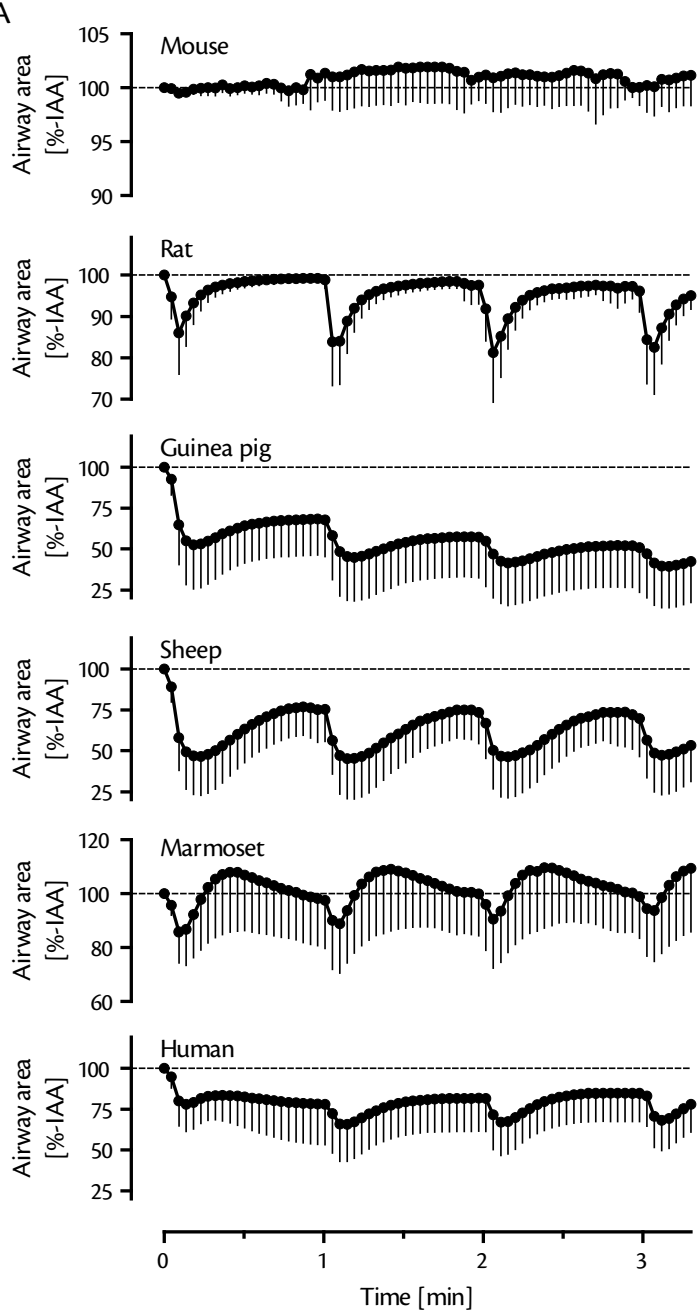

B

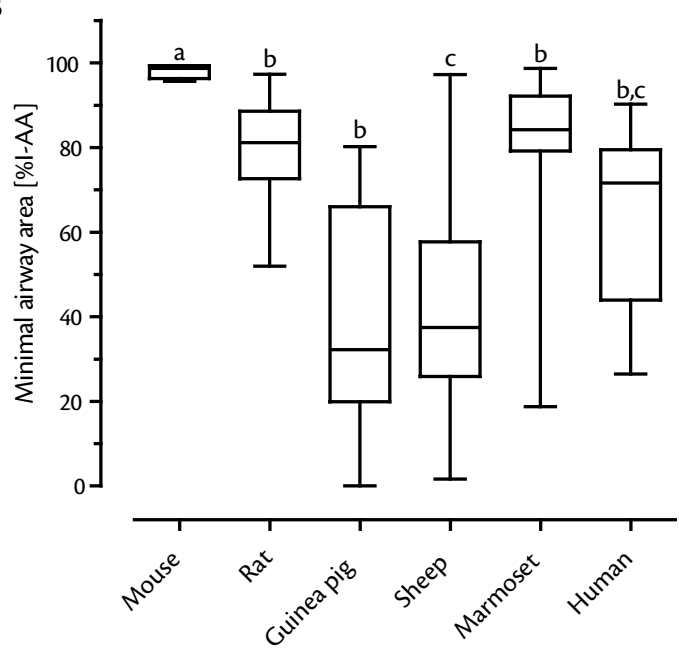

C

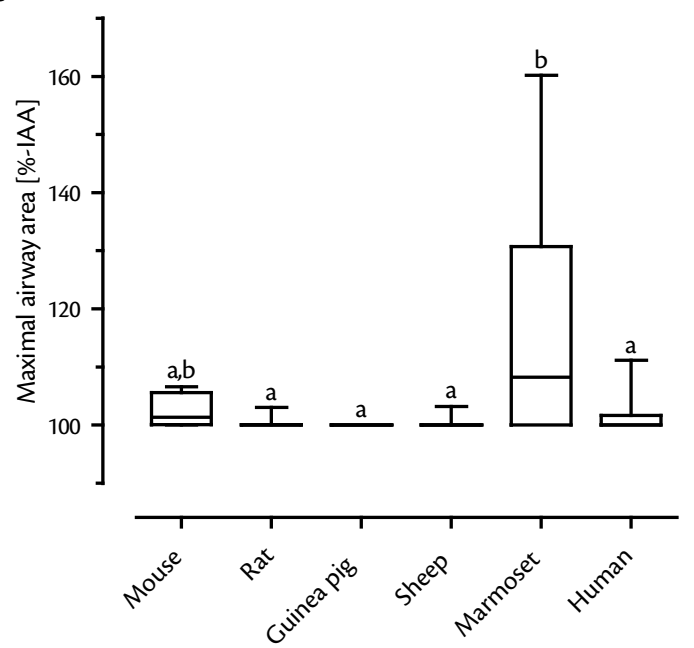

Figure 28: Airway response of PCLS from distinct species to EFS. PCLS from indicated species were stimulated repeatedly at $F=50 \mathrm{~Hz}, B=1 \mathrm{~ms}, A=200 \mathrm{~mA}, T W=2.5 \mathrm{~s}, T R=60 \mathrm{~s}$ for $3.3 \mathrm{~min}$, i.e. each $P C L S$ received $4 \times \mathrm{EFS}$. A. The time course of airway area in successive EFS trains is depicted as mean - SD for each species. The statistical evaluation based on the minimal (B) and maximal airway area (C) is shown as Box and Whiskers plots. Groups not indicated by the same letter are significantly different at $\mathrm{p}<0.05$ according to the Steel-Dwass comparison test. 
figure 28. Airways from mice did not respond in the range of the EFS conditions studied here even in the presence of neostigmine (Minimal airway area: (98.2 \pm 0.9$) \%-I A A$ (control) vs. $(94.3 \pm 2.2) \%-I A A$ (neostigmine); mean \pm SEM; $n=4 ; p=0.1581$ in $t$-test). However, the application of substantially higher frequencies or pulse durations led to airway contractions also in mouse PCLS, which were blocked by magnesium indicating neural origin (figure 29). PCLS from rats and marmoset contracted reversibly by about $20 \%$ (figure 28 A, B). Airways in PCLS from guinea pigs, sheep and human contracted strongest by about 40\%-IAA - 60\%-IAA and did not revert to the initial area during the one minute interval until the next electric impulse was applied (figure $28 \mathrm{~A}, \mathrm{~B}$ ). Marmoset airways showed a unique behaviour in that contraction was followed by relaxation that exceeded the original airway caliber ((116 \pm 18$) \%-I A A$; figure $28 \mathrm{C})$.
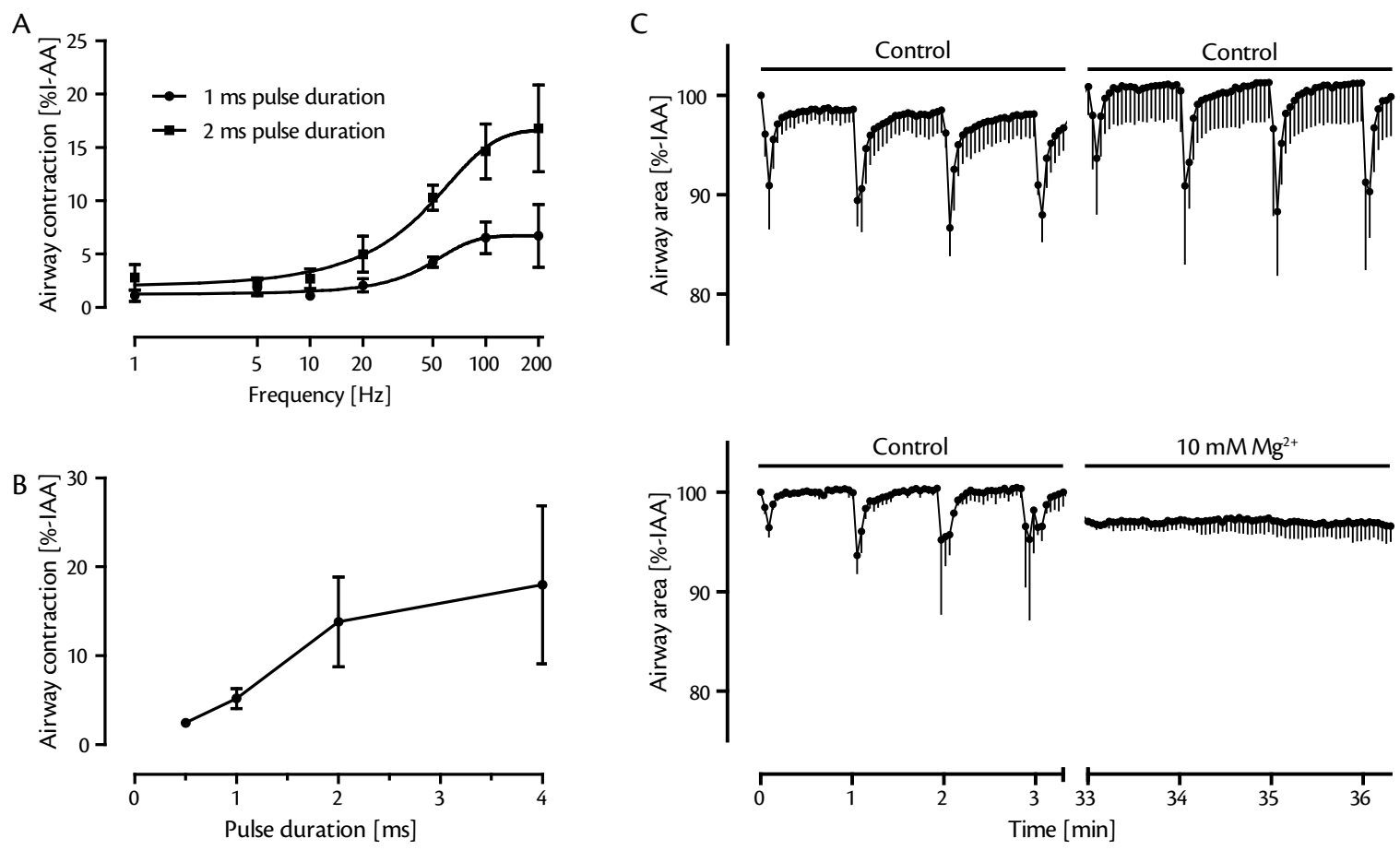

Figure 29: Electrically activated airway contractions in murine PCLS require higher electric fields and remain neuronally-induced. A. Frequency-response curve: The frequency $(F)$ was increased from $1 \mathrm{~Hz}$ to $200 \mathrm{~Hz}$, while the other EFS settings were kept constant at $B=1 \mathrm{~ms} / 2 \mathrm{~ms}, A=200 \mathrm{~mA}$ and TW $=2.5 \mathrm{~s}$. B. Pulse duration was increased in EFS of murine PCLS from $0.5 \mathrm{~ms}$ to $4 \mathrm{~ms}$, while the other parameters were kept constant at $F=50 \mathrm{~Hz}, \mathrm{~A}=200 \mathrm{~mA}$ and $T W=2.5 \mathrm{~s}$. C. PCLS from mouse were stimulated repeatedly at $\mathrm{F}=50 \mathrm{~Hz}, \mathrm{~B}=2 \mathrm{~ms} \mathrm{~A}=200 \mathrm{~mA}$ and $\mathrm{TW}=2.5 \mathrm{~s}$ each minute for $3.3 \mathrm{~min}$. Upper panel: Control stimulations, each EFS train was conducted without pharmacological interference. Lower panel: $10 \mathrm{mM}$ magnesium $\left(\mathrm{Mg}^{2+}\right)$ was added 30 min prior to the second EFS train. Magnesium was able to block airway responses indicating specific neurally-induced bronchoconstriction. Data are shown as mean \pm SD.

Analyzing the frequency-response curves (figure 30) revealed the following order of species sensitivity to EFS: sheep $\left(\mathrm{EF}_{50}=(0.5 \pm 0.2) \mathrm{Hz}\right)>$ guinea pig $\left(\mathrm{EF}_{50}=(7.0 \pm 1.2) \mathrm{Hz}\right)=$ human $\left(\mathrm{EF}_{50}=(8.1 \pm 0.8) \mathrm{Hz}\right)>\operatorname{rat}\left(\mathrm{EF}_{50}=(16.5 \pm 0.8) \mathrm{Hz}\right)$. The $\mathrm{EF}_{50}$ values for humans and guinea pigs did not differ statistically. The $\mathrm{EF}_{50}$ of marmoset was $(22.8 \pm 7.1) \mathrm{Hz}$ but the frequency-response curve neglects the relaxant response and therefore the $\mathrm{EF}_{50}$ appears higher than without relaxation. 


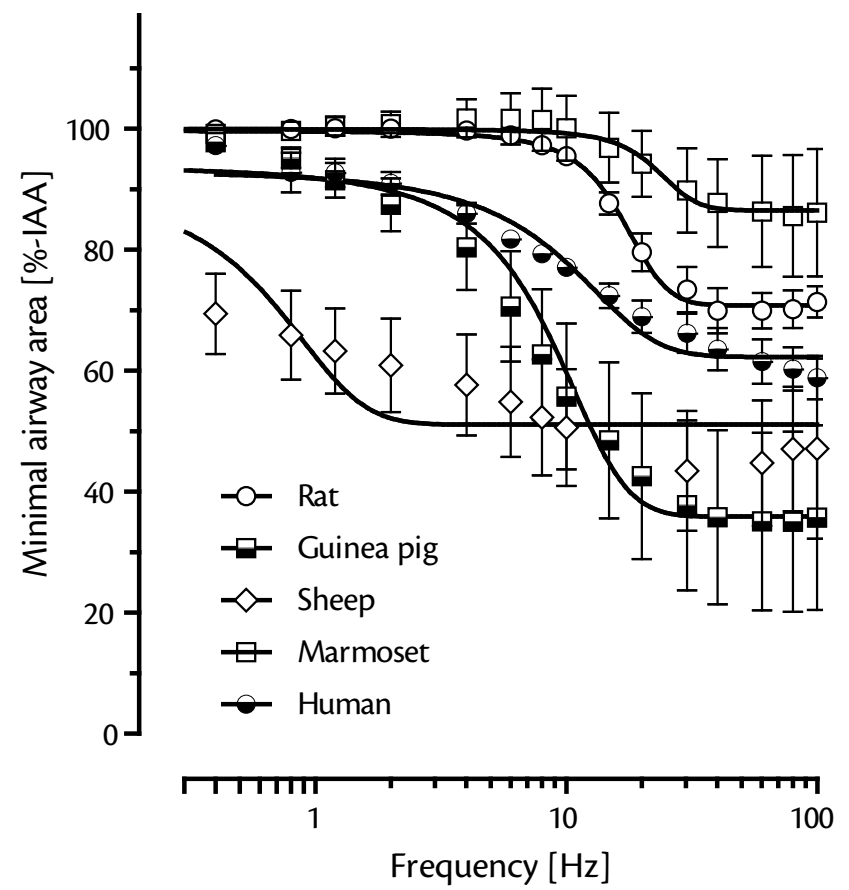

Figure 30: Frequency dependence across species. Frequency-response curves were performed on PCLS from the species indicated. Except for the frequency (F), which was increased from $0.4 \mathrm{~Hz}$ to $100 \mathrm{~Hz}$ after a single train at the distinct frequency each minute $(T R=60 \mathrm{~s})$, EFS settings were kept constant at $B=1 \mathrm{~ms}$, $A=200 \mathrm{~mA}$ and TW $=2.5 \mathrm{~s}$. Sigmoidal curves, in which the top was constrained to $100 \%$ of the initial airway area $[\mathrm{IAA}]$, were plotted and frequencies for the half-maximal response $\left(\mathrm{EF}_{50}\right)$ were determined: rat (16.5 \pm 0.8$) \mathrm{Hz}$, guinea pig $(7.0 \pm 1.2) \mathrm{Hz}$, sheep $(0.5 \pm 0.2) \mathrm{Hz}$, marmoset $(22.8 \pm 7.1) \mathrm{Hz}$, human $(8.1 \pm 0.8) \mathrm{Hz}$. Except for the guinea pig, $\mathrm{EF}_{50}$ values from all species were significantly different from the human $\mathrm{EF}_{50}$. Data are shown as mean \pm SEM; $n=6$ for rat, $n=5$ for guinea pig, $n=4$ for sheep, $n=4$ for marmoset and $n=4$ for human.

\subsubsection{Evidence for neural activation}

Magnesium competes at neural calcium channels ( $\mathrm{N}$-type calcium channels) and thus prevents

Table 5: EFS-induced airway contractions are neurally mediated.

\begin{tabular}{|c|c|c|c|c|}
\hline \multirow[t]{2}{*}{ Species } & \multicolumn{2}{|c|}{ EFS } & \multicolumn{2}{|c|}{$\begin{array}{c}\text { Methacholine } \\
\text { Minimal airway area [\%-IAA] }\end{array}$} \\
\hline & w/o $\mathrm{Mg}^{2+}$ & $10 \mathrm{mM} \mathrm{Mg}^{2+}$ & w/o $\mathrm{Mg}^{2+}$ & $10 \mathrm{mM} \mathrm{Mg}^{2+}$ \\
\hline Rat & $19.0 \pm 8.8$ & $1.4 \pm 0.6^{* *}$ & $13.5 \pm 15.1$ & $30.0 \pm 11.5$ \\
\hline Guinea Pig & $44.3 \pm 37.5$ & $3.9 \pm 4.1$ * & $0.0 \pm 0.0$ & $7.3 \pm 8.5$ \\
\hline Sheep & $54.4 \pm 22.5$ & $7.6 \pm 8.3^{\# \#}$ & $10.1 \pm 10.7$ & $18.4 \pm 20.0$ \\
\hline $\begin{array}{l}\text { Marmoset } \\
\text { (Con./Rel.) }\end{array}$ & $\begin{array}{c}46.0 \pm 20.9 \\
(28 \pm 27 / 18 \pm 20)\end{array}$ & $\begin{array}{c}13.2 \pm 9.0^{\# \#} \\
(11 \pm 11 / 2 \pm 3)\end{array}$ & $39.0 \pm 25.3$ & $38.2 \pm 16.1$ \\
\hline Human & $36.8 \pm 23.1$ & $18.9 \pm 19.0^{+}$ & $32.2 \pm 23.2$ & $35.4 \pm 20.2$ \\
\hline
\end{tabular}

Magnesium competes with calcium at the terminal synapse and prevents the release of neurotransmitters resulting in a neuromuscular block (40; 53). The muscarinic receptor agonist methacholine was used to confirm that $\mathrm{Mg}^{2+}$ did not affect airway smooth muscle directly. Con., contraction; EFS; electric field stimulation; IAA, initial airway area; $\mathrm{Mg}^{2+}$, magnesium; Rel., relaxation; w/o, without; ${ }^{*}, \mathrm{p}<0.05$; ${ }^{* *}, \mathrm{p}<0.01$ in Mann-Whitney test; ${ }^{* \#}, \mathrm{p}<0.01$ in $\mathrm{t}$-test; ${ }^{\dagger} \mathrm{p}<0.05$ in paired $\mathrm{t}$-test. 
neural responses, but not those acting directly on smooth muscle cells (40; 53). EFS performed in the presence of $10 \mathrm{mM}$ magnesium inhibited airway reactions in all species, but did not alter responses to exogenously added methacholine (table 5) demonstrating that the EFS-induced responses depended on neural transmission in all species.

\subsubsection{Cholinergic antagonism}

The muscarinic antagonist atropine significantly reduced airway contraction in repeated EFS of rat, sheep, marmoset and human PCLS (figure 31). Of note, in the case of rat PCLS the acetylcholine esterase inhibitor neostigimine was used to augment the response. In guinea pigs, PCLS had to be pre-incubated with atropine before the first stimulation in order to demonstrate cholinergic airway innervation (figure $33 \mathrm{C}, \mathrm{D}$ ).

A

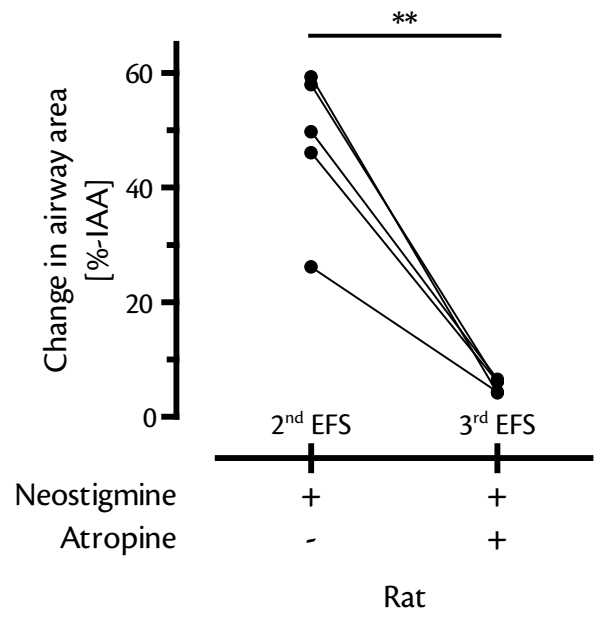

C

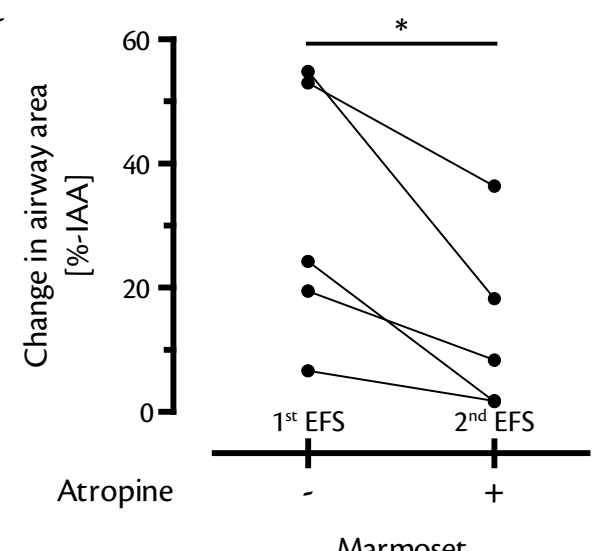

B

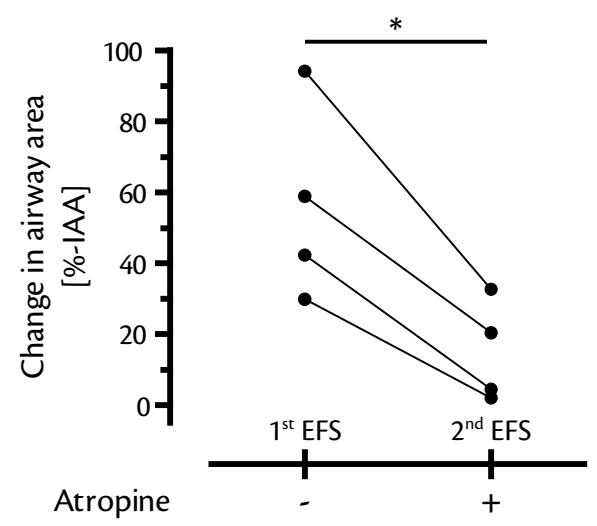

Sheep

D

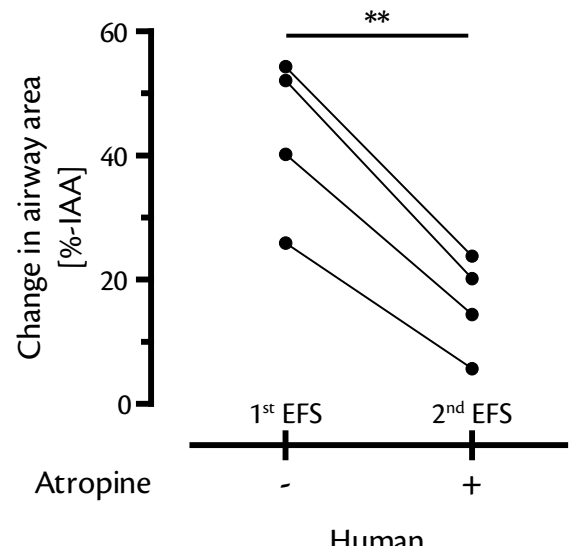

Figure 31: Cholinergic responses in EFS-triggered airway contraction. PCLS from either rats (A), sheep (B), marmosets (C) or human (D) were stimulated repeatedly at $F=50 \mathrm{~Hz}, \mathrm{~B}=1 \mathrm{~ms}, \mathrm{~A}=200 \mathrm{~mA}$, TW $=2.5 \mathrm{~s}$, $T R=60 \mathrm{~s}$ for $3.3 \mathrm{~min}$, i.e. $4 \times \mathrm{EFS}$. As control, the first stimulation ( $\left.1^{\mathrm{st}} \mathrm{EFS}\right)$ was always carried out without any cholinergic interference. In subsequent EFS separated by at least $15 \mathrm{~min}$ to the preceding stimulation, neostigmine and/or atropine were added to augment or inhibit, respectively, cholinergic airway contraction. *, $p<0.05$ and **, $p<0.01$ for atropine vs. no atropine (paired t-test). The guinea pig data are shown in figure 33. 


\subsubsection{Activation by capsaicin}

Neurally triggered airway contraction may not be restricted to cholinergic innervation but can also be due to eNANC innervation. Therefore, the effect of capsaicin was studied.

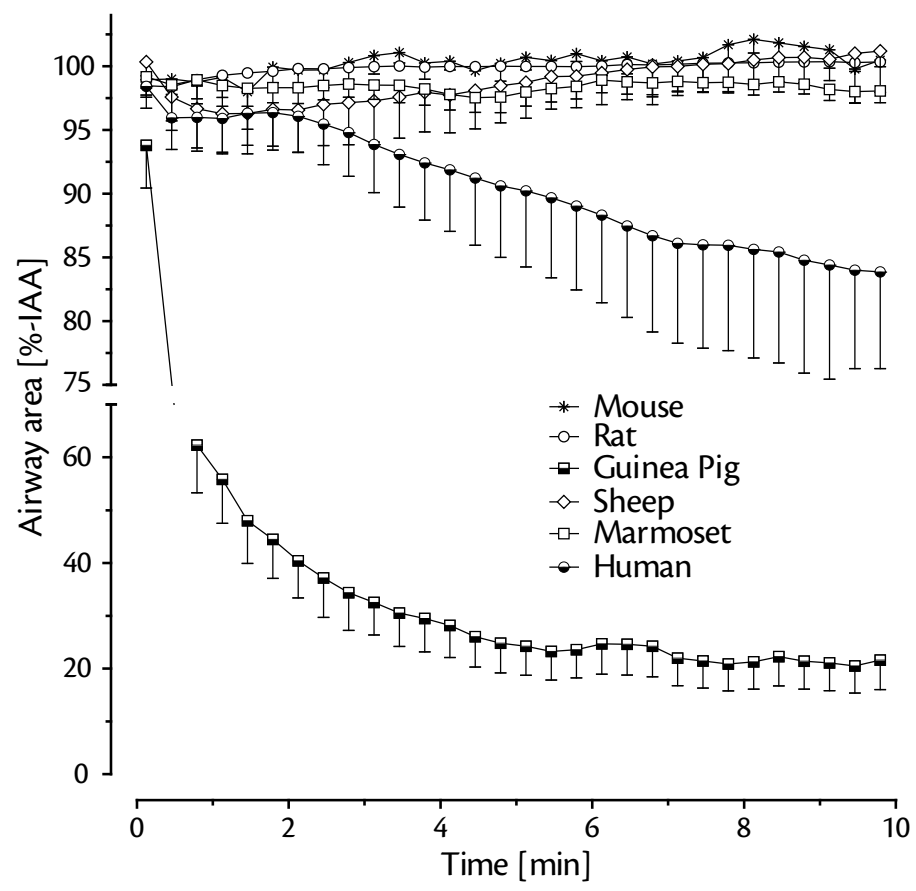

Figure 32: Effect of capsaicin on airway contraction in PCLS. Capsaicin was applied to PCLS from the species indicated at a concentration of $10 \mu \mathrm{M}$ and airway contraction was monitored. Data are shown as mean -SEM; $n=3$ for mouse, $n=10$ for rat, $n=9$ for guinea pig, $n=4$ for sheep, $n=3$ for marmoset and $\mathrm{n}=5$ for human.

Capsaicin induced strong airway contractions in PCLS from guinea pigs and moderate contractions in human PCLS (figure 32); of note, two out of five human lungs did not respond to capsaicin. In mouse, rat, sheep and marmoset capsaicin did not affect airway tone (figure 32).

\subsubsection{Role of TRP-channels in neurally evoked bronchoconstriction}

To further characterize the strong response to capsaicin in guinea pigs, PCLS were incubated with the unspecific TRP-channel blockers SKF96365 or Ruthenium red before stimulation by capsaicin. Both inhibitors significantly reduced the response to capsaicin (figure 33). Since in guinea pigs airways did not relax back to the initial airway area after an EFS (figure 28), even if recovery periods were prolonged, a non-cholinergic mechanism appeared likely. This was studied by the application of Ruthenium red and SKF96365 during EFS of guinea pig PCLS. As PCLS could not be stimulated repeatedly due to the lack of relaxation, $P C L S$ were grouped and incubated with the inhibitors prior to the first stimulation. Atropine, Ruthenium red and SKF96365 were all effective in blocking the response compared to the control group, although they failed to abolish the response completely (figure 33). 
A

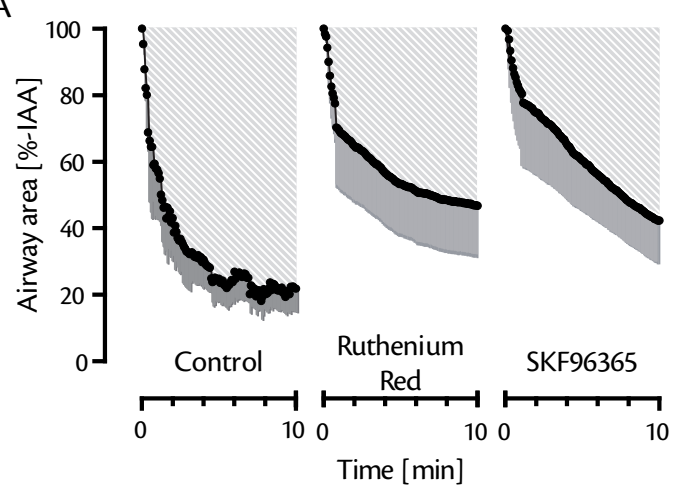

C

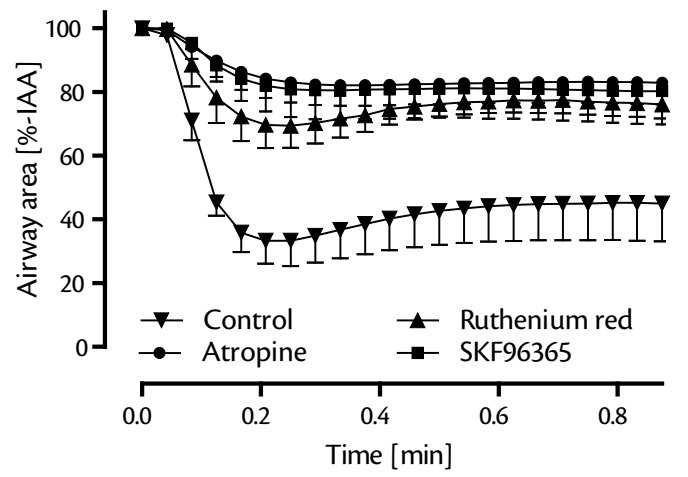

B

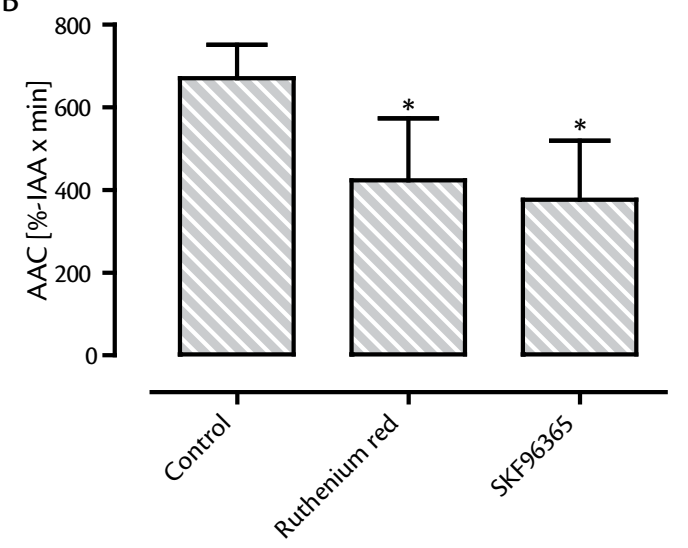

$\mathrm{D}$

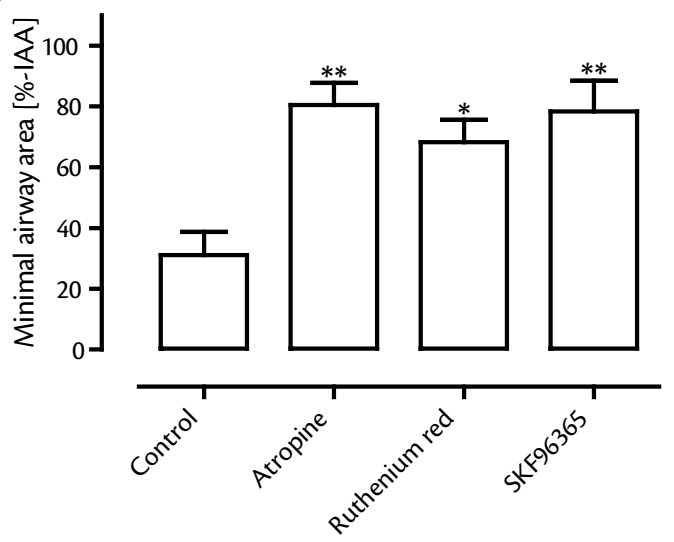

Figure 33: Characterization of peripheral airway innervation in guinea pigs. A. Capsaicin was added to precision-cut lung slices (PCLS) at a concentration of $10 \mu \mathrm{M}$, either after 15 min pre-incubation with an unspecific transient receptor potential (TRP) channel blocker (Ruthenium red, SKF96365) or without pre-incubation (control). Data are shown as mean - SEM; $n=5.100 \%$ of the initial airway area (IAA) was considered as baseline and the area above the curve (AAC), i.e., the area enclosed by the baseline and the curve, is depicted as shading. B. Statistical analysis was performed on the AAC calculated from panel A. Data are shown as mean $+\mathrm{SEM} ; \mathrm{n}=5 ;{ }^{*}, \mathrm{p}<0.05$ in linear mixed model analysis followed by the Tukey test. C. Electric field stimulation ( $E F S ; F=50 \mathrm{~Hz}, B=1 \mathrm{~ms}, A=200 \mathrm{~mA}, \mathrm{SD}=2.5 \mathrm{~s}$ ) was carried out on PCLS either after preincubation with TRP channel blockers, the muscarinic inhibitor atropine or without pre-treatment (control) and the airway responses are depicted as mean $-\mathrm{SEM} ; n=5-6$. D. The minimal airway area obtained in the EFS of PCLS was used in statistical analysis showing an effect for all antagonists. Data are shown as mean + SEM; $\mathrm{n}=5-6 ;{ }^{*}, \mathrm{p}<0.05$ and ${ }^{* *}, \mathrm{p}<0.01$ for the comparison to the control group in the Tukey multiple comparison test.

\subsubsection{Effect of propranolol and L-NAME in EFS of PCLS from marmoset}

As noted above, in marmosets the airway caliber enlarged following electric stimulation suggesting adrenergic or iNANC responses. To examine their contributions, EFS was performed subsequently to a control train in the presence of the $\beta$-blocker propranolol, the NO synthase inhibitor L-NAME or both together. Each antagonist alone was unable to significantly reduce the relaxant response, but the combination of both was effective (figure 34). Figure 34 suggests that propranolol alone might also have been effective; however the statistical power was probably not sufficient; a statistical 

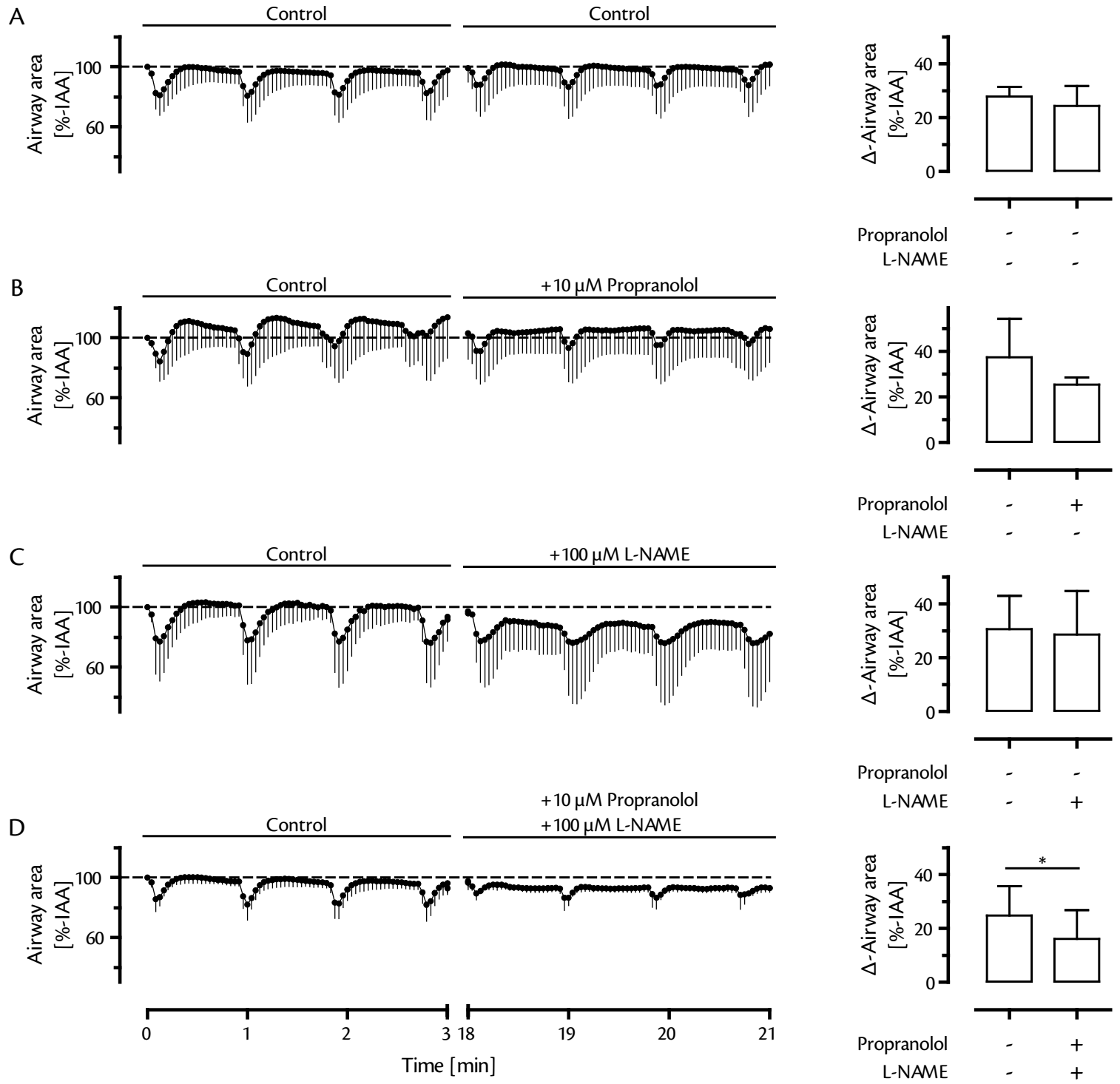

Figure 34: Airway relaxation in marmosets is due to adrenergic and inhibitory nonadrenergic noncholinergic (iNANC) innervation. The same PCLS was stimulated repeatedly $(F=50 \mathrm{~Hz}, B=1 \mathrm{~ms}$, $\mathrm{A}=200 \mathrm{~mA}, \mathrm{TW}=2.5 \mathrm{~s}, \mathrm{TR}=60 \mathrm{~s}$ ): the entire time course for a single experiment with one PCLS is shown on the abscissa. The time course of airway area in successive EFS trains ( 4 XEFS in $3.3 \mathrm{~min}$ ) is depicted on the left and the statistical evaluation based on the difference between the maximal and minimal airway area ( $\triangle$-airway area) during the respective train is shown as bar graphs on the right. $\mathbf{A}$. The results of EFS without any addition to the incubation medium. B. The results from EFS experiments in the presence of the adrenergic $\beta$-blocker propranolol. C. The results from EFS experiments in the presence of the NO synthase inhibitor L-NAME. D. The results from EFS experiments in the presence of the combination of propranolol and L-NAME. Data are shown as mean - SD (left) or mean + SD (right); ${ }^{*}, \mathrm{p}<0.05$ in one-tailed paired t-test.

power analysis suggested that 9 independent experiments might be required to achieve statistical significance. However, as marmosets are non-human primates and in order to save animals, we refrained from further experiments to clarify this issue. Regarding L-NAME it is remarkable, that in its presence airway recovery to baseline values was prevented (figure $34 \mathrm{C}, 2^{\text {nd }}$ train), suggesting a role of NO in this process. 


\section{Discussion}

Application of an electric field to PCLS resulted in airway contractions that were inhibited by magnesium. Therefore, in PCLS bronchoconstriction triggered by EFS is neurally induced implying that nerve endings remain intact in PCLS. In rats, neural responses are mainly cholinergic and decrease with decreasing airway size. PCLS from allergic rats were neither hyperresponsive to EFS nor to methacholine indicating that increased release of acetylcholine from postganglionic parasympathetic nerves does not occur and that acetylcholine receptors and the postreceptor transduction mechanisms are not affected by allergen exposure in rat. Finally, the EFS was adopted for PCLS from mice, guinea pigs, sheep, marmosets and humans. Cholinergic airway responses were found for guinea pigs, sheep, marmosets and humans. Furthermore, guinea pig and human PCLS were activated by capsaicin showing eNANC responses. The EFS-induced airway dilation in PCLS from marmosets was blocked by propranolol and L-NAME pointing to iNANC nerves in this species.

\subsection{Design and development of EFS-setup on the basis of rat PCLS}

Viable PCLS have been established as a useful tool to study airway tone, pulmonary vascular responses, ciliary beating as well as immunological and toxicological properties of the lung (34; 68; 111; 112; 188; 189). This study established neural stimulation of PCLS. EFS-induced bronchoconstriction is consistent, reproducible, sensitive to anticholinergics and enhanced by an acetylcholine esterase inhibitor in rats. Ipratropium was more potent to block EFS-induced cholinergic airway contraction than atropine or 4-DAMP. The effect of the EFS was strongest in the larger airways and appeared to be mediated by synaptic release of acetylcholine. This model will useful to further study mechanisms of neural airway control and cholinergic AHR in rats and other species.

\subsubsection{General EFS-setup in PCLS}

The extent of the EFS-induced contractions of 15\%-IAA in large airways roughly corresponds to contractions elicited by $330 \mathrm{nM}$ of the metabolically stable acetylcholine derivative methacholine (figure $8 \mathrm{~B}$ in (111)), suggesting that this may be the effective acetylcholine-concentration in the synaptic cleft following presynaptic discharge. These findings indicate that neurally-induced bronchoconstriction can regulate airway tone, but is unlikely to lead to complete airway closure as shown for instance for allergen provocation (189). Comparing the present findings to other studies it should be noted that the data were not normalized to contractions induced for example by $\mathrm{KCl}$, a procedure which may overstate the real magnitude of the effects. Normalization to receptor agonists (e.g. methacholine) was omitted throughout the study as it has been previously shown that responsiveness of small airways is stronger to methacholine and other stimuli $(111 ; 112 ; 188$; 
189). In such a situation, normalization would distort the results. The EFS-induced contraction by approximately $15 \%$-IAA was strongly potentiated by inhibition of the acetylcholine esterase (figure 12). These findings indicate that in PCLS cholinergic synapses remain intact, as they respond to electrical currents, possess active acetylcholine esterase (see experiments with neostigmine) and acetylcholine-receptors (see experiments with atropine) and recycle acetylcholine in a normal fashion (otherwise repetitive stimulations would not have been possible).

EFS was applied to stimulate neuronal cells, but, depending on the frequency and duration of the electric stimulus, direct contraction of smooth muscle cells may also occur (45). To exclude such an unspecific direct stimulation of voltage-gated channels on ASM, the effect of magnesium was examined. At high concentrations, magnesium prevents voltage-induced calcium entry and thereby the release of neurotransmitters from nerve terminals $(40 ; 53)$. Since magnesium completely prevented the bronchoconstriction induced by EFS, but not by methacholine, it is concluded that in the present setup the EFS acts only on nerve endings and not on ASM (figure 15).

\subsubsection{Relevance of the basic EFS conditions}

For organ bath experiments, Wang and colleagues (183) systematically studied the influence of pulse duration $(0.5-3 \mathrm{~ms})$, muscle preload $(2-20 \mathrm{~g})$, voltage $(5-20 \mathrm{~V})$ and frequency $(0.5-16 \mathrm{~Hz})$ on the release of acetylcholine from equine airway cholinergic nerves, in order to provide guidelines for selecting EFS parameters in future studies. As they observed an increasing acetylcholine release from 2.1 - $18.6 \mathrm{pmol} / \mathrm{g}_{\text {tissue-wet-weight }} / \mathrm{min}$ with increasing frequencies $(2-16 \mathrm{~Hz})$, frequencies were analyzed in the present study by steadily increasing them while the other stimulation parameters were kept constant. This resulted in a sigmoidal frequency-response curve for the airway area change (figure $11 \mathrm{~A}$ ) plateauing at $\mathrm{F}=50 \mathrm{~Hz}$, somewhat above the frequency observed by Wang and colleagues (183) $(>8 \mathrm{~Hz}$ ). The most likely explanation for these differences is interspecies variability, as PCLS from other species (e.g. sheep) responded already at lower frequencies (figure 30) and our $\mathrm{EF}_{50}$ of $(16.7 \pm 4.9) \mathrm{Hz}$ is almost identical to the $(19.3 \pm 4.3) \mathrm{Hz}$ reported by Gonzalez and Santana (61) on isometric tension studies of rat tracheal smooth muscle. In addition they also found a plateau phase above $40 \mathrm{~Hz}$ (61), whereas the $\mathrm{EF}_{50}$ of $4.4 \mathrm{~Hz}$ in Wang's and colleagues' (183) study of horse airways was markedly lower. To put these figures into proportion, the maximal frequency that allows motoneurons to depolarize during repetitive stimulation is $500-1000 \mathrm{~Hz}$ (84). It should also be noted that Mitchell and colleagues (119) showed relatively high frequencies of action potentials (peak frequency of $10-13 \mathrm{~Hz}$ ) for feline tracheal parasympathetic ganglion cells during normal breathing. Also other factors such as electrode geometry and the ionic strength of our cell culture medium have to be taken into account, as the inner electric field strength depends on the conductivity of the medium and on the current density, which in turn depends on a shape factor, the frequency and the external electric field strength. Taken together, the frequencies that were applied in rat PCLS appear reasonable. 
With respect to pulse duration it was found, similar to Wang and colleagues (183), that durations of $B \geq 1$ ms were needed to reach a plateau response. In all the experiments (except figures 11 and 24) a current density of $A=200 \mathrm{~mA}$ was used, which was the only current that gave a reproducible contraction; since $200 \mathrm{~mA}$ is the maximum current output of the HSE-stimulator II, higher currents could not be studied. Another important factor influencing ASM contraction is train width: in the present study maximal contraction was achieved at $T W \geq 10 \mathrm{~s}$ (figure 11), similar to Bosse and colleagues $(25 ; 26)$, who used a maximum airway contraction at TW $=9 \mathrm{~s}$ when examining the influence of increased tone (by acetylcholine) on the mechanical properties of ASM. However, at TW = $10 \mathrm{~s}$ airway contractions took longer to reverse and, more importantly, could not be blocked completely by magnesium, indicating that the electrical stimulation was more unspecific at this train width and activated both nerve endings and ASM. In order to avoid direct activation of ASM TW $=2.5 \mathrm{~s}$ was used, but a TW $=5 \mathrm{~s}$ would also seem appropriate. Since a frequency of $F=50 \mathrm{~Hz}$ was used, a train width of TW $=2.5 \mathrm{~s}$ implies a series of 125 single pulses that were separated by a recovery phase of about one minute (exactly $57.5 \mathrm{~s}$ ). The duration of this phase allowed recovery under normal conditions (figure 10 and figure 12 upper panel), but was too short when neostigmine was present. However, as the maximum contraction was always reproducible, it is concluded that the lack of complete recovery in figures 12,15 and 17 is not a major limitation. It is important though to note that the different stimulation trains were separated by at least $15 \mathrm{~min}$ to assure full recovery of the ASM to its physiological ground state before the next experimental setting was examined. In addition this period was used to equilibrate the slices with the pharmacological agents to be studied next.

\subsubsection{Cholinergic neuronal response in PCLS}

Acetylcholine release from parasympathetic nerve terminals regulates the airway tone in various species including human (14; 32; 61). The enhanced airway contraction after addition of neostigmine, an acetylcholine esterase inhibitor (figure 12), indicates locally increased concentrations of acetylcholine at the neuroeffector junction (104; 114). Atropine, an unspecific inhibitor of muscarinic receptors, almost completely inhibited the neuronal cholinergic contraction in PCLS, even in the presence of neostigmine (figure 12). Atropine most probably prevents ASM contraction by blockade of the $M_{3}$ (and $M_{2}$ ) receptors on the postsynaptic membrane, such that the $M_{3}$ receptors cannot initiate the contraction via the $\mathbb{P}_{3}$ pathway $(14 ; 21 ; 115 ; 141)$. The small residual bronchoconstriction in the presence of $10 \mu \mathrm{M}$ atropine may be explained by the blockade of presynaptic muscarinic receptors by atropine, preventing the autoinhibitory effect of acetylcholine release $(14 ; 21)$. Furthermore, the release of SP might potentiate the release of acetylcholine from cholinergic nerves (14) and trigger the release of bronchoconstrictive tachykinins from excitatory NANC (49). 
Having proven the cholinergic mechanism in EFS of PCLS from rat, the method was tested against distinct $M$ receptor antagonists at different concentrations to evaluate its potential for pharmacological discrimination of drugs. Atropine, ipratropium and 4-DAMP were used as representative substances - the first two being unspecific $M$ receptor antagonists and the latter a selective $M_{3}$ receptor antagonist (168). Since repeated stimulations were reproducible (figure $13 \mathrm{~A}$ ) an increase of the inhibitor concentration in consecutive EFS trains is feasible and a diminished response can be attributed to the effect of the antagonist and not to fatigue of the ASM. Consistently, increasing concentrations of atropine, ipratropium or 4-DAMP inhibited EFS-induced airway contractions. Calculated $\log \mathrm{IC}_{50}$ values (figure 14), revealed ipratropium $(-10.0 \pm 0.4)$ being more potent to inhibit EFS evoked airway responses than atropine $(-8.8 \pm 0.4)$ or 4-DAMP (-8.4 \pm 0.3$)$. So far only one other study compared the effect of atropine, ipratropium and 4-DAMP in parallel in the same EFS setup, but on twitch responses of guinea pig tracheal ring preparations (168). However, equal $\log I \mathrm{IC}_{50}$ of $-8.63 \pm 0.04,-8.64 \pm 0.04$ and $-8.61 \pm 0.05$ were found for atropine, ipratropium and 4-DAMP, respectively, which except for ipratropium were similar to those determined for rats in this study. Species differences and differences in setup may account for the discrepancy. Therefore, the obtained $\log I \mathrm{IC}_{50}$ values herein seem reasonable and the EFS of PCLS is a valuable tool to characterize and discriminate potential anticholinergic drugs.

\subsubsection{Reactivity of different airway generations}

PCLS have previously been used to study airway responses along the bronchial tree. Smaller airways are more responsive to methacholine, serotonin, the TP receptor agonist U46619 and allergens in lungs of different species (112; 188; 189). Endothelin-1 contracted airways independent of their size (112), indicating that smaller airways are not more sensitive in principle. In the present work it was shown that smaller airways are less sensitive to EFS (figure 16). This observation can be explained most likely by the decreasing innervation of the bronchial tree towards the peripheral regions (14). Thus, parasympathetic bronchoconstriction in the airways appears to be balanced by inversely arranged innervation and acetylcholine-responsiveness: rich innervations with low acetylcholine-responsiveness in large airways opposed to more sparse innervations with high sensitivity in smaller airways.

\subsubsection{Role of the TP receptor in neuronal activation}

Subthreshold doses of TP receptor agonists (e.g. U46619), i.e. doses which do not evoke airway contraction via the TP receptor themselves, have been shown to increase parasympathetic cholinergic bronchoconstriction in mice (as measured by lung resistance) (7), guinea pig (lung resistance) (176) and dogs (isometric tension) $(4 ; 167)$. Interestingly, in the study of Takata and colleagues (167) the effect was only found for bronchial smooth muscle, whereas tracheal smooth muscle was unaffected. This suggests regional differences in airway responsiveness, with smaller 
airways being more susceptible to TXA. Additionally, Saroea and colleagues (150) suggested U46619-induced bronchoconstriction via acetylcholine release in asthmatic subjects. Moreover, rat tracheal prostanoid synthesis can be stimulated by activation of $M$ receptor-linked calcium mobilization (78). These observations suggested a possible influence of endogenously released TXA on EFS in the present model. However, the TP receptor antagonist SQ29548 had no effect (figure 17), indicating that EFS-induced airway contractions in the rat are not influenced by TXA. This is confirmed by the observation that even a pre-contracting concentration of U46619 did not alter the response to EFS (figure 18). These observations are in line with other in vitro studies. For instance, Aizawa and Hirose (3) measured airway contraction of canine tracheal strips in response to acetylcholine or EFS either in presence or absence of $\mathrm{PGF}_{2 \alpha}$ or stable $\mathrm{TXA}_{2}$ and found no difference. They therefore suggested that the interaction of TXA and cholinergic pathways depends on stimulation of vagal sensory endings and activation of the reflex pathway in vivo. This conclusion is supported by studies from Underwood and colleagues (176), who discriminated strictly between in vitro and in vivo responses to $\mathrm{PGD}_{2}$ and $9 \alpha, 11 \beta \mathrm{PGF}_{2}$. In vivo, atropine antagonized $\mathrm{PG}$ increased airway resistance similar to the TP receptor antagonist SK\&F 88046, whereas in vitro (tracheal strip) only TP receptor antagonists were effective. Thus, our findings suggest that the reflex arc is not complete in PCLS.

An interaction of TP and $M$ receptor pathways may not be limited to prejunctional nerves, but may also occur at the ASM itself. Therefore, cumulative concentration-response curves were conducted with U46619 in presence or absence of atropine and no difference was found (figure 19). This finding is in accordance with in vitro contraction of tracheal strips from guinea pigs, where comparable concentration-response curves for TP receptor agonists (U44069, PGD ${ }_{2}$, and $9 \alpha, 11 \beta \mathrm{PGF}_{2}$ ) in the presence or absence of atropine were obtained (176). In contrast, Allen and colleagues (7) observed a notable reduction in U46619- (50 - $500 \mathrm{nM}$ ) evoked murine tracheal ring constriction at $1 \mu \mathrm{M}$ atropine. They explain their findings by an increased sensitivity of the $\mathrm{ASM} \mathrm{M}_{3}$ receptor to its cognate physiological ligand acetylcholine after stimulation of the ASM TP receptor by TXA. However, their model presupposes the existence of an ASM basal tone and constitutive release of acetylcholine in the unprovoked airways. Therefore, their data are not conflicting to the results herein, as it is assumed that there is no or negligible basal release of acetylcholine in the present system, since neostigmine had no effect in un-stimulated PCLS (figure 12). Nonetheless, possible species differences can easily be studied with the help of PCLS $(34 ; 111 ; 145 ; 188 ; 189)$.

\subsubsection{Comparison to other in vitro models}

Previously, EFS has been almost exclusively studied in the organ bath using trachea, bronchi and parenchymal strips. In comparison, PCLS have both advantages and disadvantages. First, organ bath studies are limited to the trachea or upper bronchi or to the very distal part of the lungs as in parenchymal strips. PCLS from rat lungs allow to study nearly all generations along the bronchial 
tree. On the other side PCLS are very thin and therefore the percentage of neurons and especially ganglia will be lower than in tracheal and bronchial preparations. Second, airway contractions in the organ bath are either isotonic or isometric, whereas they are more auxotonic (like in vivo) in PCLS. The airways remain embedded in the parenchyma that exhibits tethering forces on the airways increasing with contraction. These tethering forces became visible when using a Teflon ring that weighs on the outer parts of the slice, so that the tethering forces increase during airway contractions. In the absence of the Teflon ring the whole slice contracted and no relaxation occurred, implying the lack of sufficient tethering forces (figure 10). So presumably, the Teflon ring acts like the attachment of the lung tissue to larger vessels and airways. Third, the PCLS setup requires only small buffer volumes of $1 \mathrm{~mL}$ and volume could be further decreased to $0.4 \mathrm{~mL}$, which is advantageous when expensive substances will be tested. Fourth, PCLS allow to analyze airway responses throughout the bronchial tree, allowing to analyze airways down to a diameter of $50 \mu \mathrm{m}(111 ; 188 ; 189)$. Thus, the present method will help to further elucidate the role and the mechanisms of small airway innervation. In contrast to classic parenchymal preparations for the study of peripheral lungs, in which force generation from different sources (ASM, vasculature) cannot be distinguished, the present model can specifically attribute contractions to airways or vessels along the entire tracheobronchial tree. And finally, the technique of PCLS can be easily transferred to other species, including human.

\subsection{Active sensitization of rats}

In the present study, only healthy animals were examined in EFS of PCLS and their peripheral airway innervation could be characterized. In the following section rats were sensitized with OVA as a representative experimental animal model of asthma and alterations in nerval responses were studied. Haemorrhages in the lung and airway contractions in response to allergen pointed to an ongoing inflammatory process and an acute allergic response, but responses to methacholine and EFS were the same in control and OVA treated animals excluding a hyperresponsive state. IgE levels were determined to prove the immune status of the animals. While OVA alone did not raise IgE levels, inactivated Bordetella pertussis organisms, given as adjuvants, were effective in increasing the IgE levels more than threefold.

\subsubsection{Sensitization scheme}

The Brown Norway rat strain was chosen for sensitization, because it presents a more pronounced IgE and inflammatory response to allergen challenges than other rat strains (87). The common features of Brown Norway rats and human asthma include high IgE levels, EAR, LAR, eosinophilia and $\mathrm{TH}_{2}$ cytokine production (87). The sensitization scheme of Brown Norway rats was based on the protocol from Glaab and colleagues (60). This group could prove an EAR and LAR in response to OVA in vivo after sensitization by two modes of measurement: noninvasive head-out body 
plethysmography and invasive determination of pulmonary conductance. Moreover, enhanced IgE levels and pronounced inflammation were detected by Glaab and colleagues (60). Therefore, the sensitization scheme promised to obtain a suitable rat model imitating acute allergic asthma.

The macroscopic observation of increased haemorrhage in sensitized animals versus the control animals (figure 20) points to an acute inflammatory process - in which vascular leakage appeared and gives evidence for an immunogenic response to OVA. Confirming this and according to Glaab's and colleagues' (60) findings, airways contracted in response to OVA in PCLS from sensitized rats, whereas no contraction was found in control animals. However, the observed contractions of (12 \pm 11$) \%$ were weak. In contrast, Glaab and colleagues (60) found a pronounced EAR to OVA in sensitized animals as both the midexpiratory flow and the pulmonary conductance dropped about more than $70 \%$ from baseline values. However, those physiological parameters may not directly correlate with the decrease in airway caliber in PCLS. Hence, a comparison with an allergic response in PCLS itself maybe more appropriate. So far, there is no data published on OVA provocation of PCLS from actively sensitized rat, which describe the extent of airway contraction exactly. However, in an abstract, Martin and colleagues (109) report of strong and sustained airway contractions to allergen in vivo and in PCLS. Surrogate quantitative data may be considered from passively sensitized PCLS, i.e. PCLS that were incubated overnight with sera from actively sensitized animals. Two studies describe the response of passively sensitized PCLS from rat to OVA $(107 ; 189)$. Wohlsen and colleagues (189), for instance, found an airway contraction of approximately $90 \%$ after allergen provocation suggesting that a nearly complete airway closure should also be possible in PCLS from actively sensitized animals. In principal, the rat is a weak allergic bronchoconstrictor and more agonist is necessary to produce airway contractions compared to doses used in guinea pigs (87).

Furthermore, the weak contraction found in the present study suggests only a low number of mast cells bearing OVA specific IgE and therefore only low mast cell degranulation. This correlates well with the unaffected IgE titers during sensitization to OVA (figure 23). IgE titers were only affected if in addition heat inactivated Bordetella pertussis organisms were used as adjuvants to induce $\mathrm{T}_{\mathrm{H}}$ 2-lymphocyte immune reaction, i.e. $\mathrm{T}_{\mathrm{H}}$ 2-lymphocytes release IL-4, which stimulates B-lymphocytes to produce IgE (30). Although IgE titers were elevated by the Bordetella pertussis adjuvants, doses of the adjuvants may be critical for a successful allergen sensitization, since the activity of the Bordetella pertussis preparation may vary and since other protocols use higher doses per animal (e.g. Kucharewicz et al. (88) $10^{10}$ organisms, Misawa et Chiba (118) $2 \times 10^{10}$ organsims) than in the present protocol ( $6 \times 10^{9}$ organisms), which was in accordance to Glaab and colleagues (60). A more sophisticated approach could therefore comprise a titration of the adjuvants to achieve IgE increases depending on the sensitization with allergen. Since the IgE ELISA determined total IgE and not OVA specific IgE and as both sensitized animals and control animals showed equal levels of IgE, a quantitative statement on OVA directed immune response cannot be 
made. However, total IgE levels were quite high even in non-sensitized animals, since they exceeded the levels found by Palmans' group (136) ((1.66 \pm 0.80$) \mu \mathrm{g} / \mathrm{mL}$ in saline treated rats vs. $(15.87 \pm 1.4) \mu \mathrm{g} / \mathrm{mL}$ in OVA sensitized rats) and Glaab's group (60) $(0.49 \pm 0.06 \mu \mathrm{g} / \mathrm{mL}$ in saline treated rats vs. $(1.61 \pm 0.59) \mu \mathrm{g} / \mathrm{mL}$ in OVA sensitized rats) by one and two orders in magnitude, respectively.

Since basic IgE levels were still high one may speculate that pre-sensitization of the animals could have occurred, in which case an increase due to OVA sensitization would hardly be visible. Presensitization is conceivable, since in Brown-Norway rats pulmonary inflammation has been observed under normal animal room environment due to ambient particles $(0.3-1 \mu \mathrm{m}$ in diameter) and the number of those particles correlated strongly with the number of eosinophils in the bronchoalveolar lavage fluid (133). However, in the present study lung tissue was reserved for PCLS preparation, which excluded the determination of eosinophils.

\subsubsection{Airway hyperresponsiveness}

$A H R$ - an exaggerated airway response to an unspecific stimulus - is a common feature of human bronchial asthma. To test whether sensitized rats exhibit an AHR, concentration-response curves were performed for methacholine on PCLS from OVA or saline challenged rats (figure 22). However, the concentration-response curves were the same in both groups indicating that there was no hyperresponsiveness to methacholine after three weeks of sensitization. This was unexpected as AHR was reported to occur in rats $(89 ; 136)$, but in these studies AHR was measured in vivo, whereas PCLS are an in vitro method. The lack of an AHR to methacholine might be explained by that fact. However, this is questionable because Martin and colleagues (109) performed a comparative study on asthmatic airway responses in rats in vivo and in vitro and found AHR to acetylcholine and adenosine. The AHR to acetylcholine in vitro only occurred if an EAR was experienced one day before in vivo. Although the rats were challenged by OVA one day before PCLS preparation, it cannot be guaranteed that an EAR took place and may therefore explain the lack of AHR in the present study. Moreover, the time schedule of sensitization could have an important impact on the occurrence of an AHR. For instance, Palmans and colleagues (136) found an increase in airway responsiveness to carbachol as measured by airway resistance if rats were challenged for two weeks by OVA in comparison to PBS-exposed animals, but not if challenge lasted 4 or 12 weeks. This concerned the concentration to produce $50 \%$ increase in baseline lung resistance. In addition to this, a left ward shift in dose response curves of carbachol was observed for animals treated two weeks with OVA compared to animals treated for 4 or rather 12 weeks with OVA (136). Since in the present study animals were exposed to OVA for three weeks, this may be a critical timeframe for whether an AHR is observable or not. The time is especially crucial with respect to airway remodelling, i.e. structural changes observed in asthmatics, and Palmans and colleagues (136) ascribe their observed difference to different states in airway remodelling. Firstly, the inner airway 
wall became thicker with two weeks of OVA challenge, which then gradually declined until the $12^{\text {th }}$ week and secondly, collagen deposition increased with prolonged OVA exposure (136). The increased thickness of the inner airway wall enhances the effect of a given ASM contraction (as elicited by a bronchcoconstrictor, e.g. carbachol and methacholine) following Poiseuille's law for laminar flow (in other words: with thicker airway wall the inner airway diameter is more reduced by the same extent of ASM contraction) and thus contributes to increased airway sensitivity, i.e. AHR (120; 136). In contrast, increased collagen deposition increases airway wall stiffness opposing airway contraction $(90 ; 136)$ and increased collagen associated with the ASM layer itself also limits airway narrowing $(29 ; 136)$.

\subsubsection{EFS of PCLS from actively sensitized animals}

During asthma AHR attributed to nerves can be due to enhanced reflex activity, increased mediator release, altered signal transmission, increased receptor-density on neurons and quantitative changes in sensory and/or motor neurons $(65 ; 143)$. Moreover, the ASM may exhibit an enhanced sensitivity to neuropeptides and tachykinins or also display an increased receptor expression (143) (chapter 1.9). To test whether nerve function is altered after three weeks of sensitization and if this is measurable in vitro, PCLS from OVA-sensitized rats were subjected to EFS and the airway response was compared to saline treated animals. However, there was no difference in the sensitivity to the four basic EFS parameters except for the current (figure 24). A shared frequencyresponse curve was found for sensitized and control animals. For the other parameters the curves of OVA exposed rats run above the curves of the saline group indicating weaker airway contraction. Hence, there is no AHR to EFS found in PCLS, but rather hyporesponsiveness, which is further confirmed by a significant reduction in bronchoconstriction to standard $E F S(F=50 \mathrm{~Hz}, B=1 \mathrm{~ms}$, $\mathrm{A}=200 \mathrm{~mA}, \mathrm{TW}=2.5 \mathrm{~s}$ ) of sensitized rats compared to control rats (figure 25). In consequence of the uncertain susceptibility of rat to sensitization and therefore heterogeneous immune state, the airway responses of all PCLS - both of sensitized and control rats - were correlated to IgE levels and confirmed lesser reactivity with higher IgE (figure 27), i.e. higher immunogenicity. In contrast, the airway response to EFS was uncorrelated to the response to allergen suggesting that sensitization of rat has no effect on neurally evoked bronchoconstriction (figure 26).

Overall, the findings contradict the expectation of an AHR, which is in contrast to literature. For instance, Braun and colleagues $(30 ; 31)$ found in two EFS studies on mouse trachea a drop in the $\mathrm{EF}_{50}$ from $4 \mathrm{~Hz}$ in PBS challenged mice to $2 \mathrm{~Hz}$ in OVA challenged mice. However, those studies were performed in another species and species differences may account for the discrepancy. Notably, a Pubmed search on the keywords: rat, airway hyperresponsiveness and electric field stimulation was performed and revealed only three results of which one deals with AHR to OVA sensitization (50). In that study, confirming the findings herein, there was no AHR to EFS developed after both acute and chronic sensitization to OVA and AHR was also not found for acetylcholine. 
It was concluded, which may also be the case here, that increased release of acetylcholine from postganglionic parasympathetic nerves does not occur and that acetylcholine receptor and the postreceptor transduction mechanisms are not affected by allergen exposure.

The observed hyporesponsiveness within the present model may result from a fibrotic remodelling of the airways resulting in an increased airway wall stiffness or affecting the ASM itself as discussed above (chapter 5.2.2) (29; 90; 136). The airways of sensitized animals have possibly been precontracted due to inflammatory mediators - thus limiting the extent of EFS-inducible airway contraction, but this issue has to be elucidated by the application of ASM relaxant agonists.

\subsection{Comparison of different species in EFS of PCLS}

In the present the study an electric field was applied to PCLS from different mammals allowing the species comparison of neuronally triggered airway responses under identical conditions. Species were heterogeneous in their response (table 6): In rats, sheep, guinea pigs, marmosets and humans, bronchoconstriction was caused by cholinergic innervation. Moreover, in guinea pig and human PCLS also activation of eNANC nerves led to airway contractions. The blockade of airway relaxation in marmosets by the inhibition of $\beta$-receptors and NO synthase points towards adrenergic and iNANC innervation in this species.

\subsubsection{General considerations on EFS of PCLS}

In the preceding part rat EFS was introduced as a means to study neural responses in PCLS and the following EFS settings were found to be effective (152): $F=50 \mathrm{~Hz}, \mathrm{~B}=1 \mathrm{~ms}, \mathrm{~A}=200 \mathrm{~mA}$ and $\mathrm{TW}=2.5 \mathrm{~s}$. The same parameters were also used to stimulate PCLS from mice, guinea pigs, sheep, marmosets and humans and evoked characteristic airway contractions in each species (figure 28), except for the mouse. The neural mediation of these responses was proven by the inhibitory effect of magnesium concentrations ( $10 \mathrm{mM}$ ) high enough to prevent voltage-induced calcium entry and the release of neurotransmitters at the nerve terminals $(40 ; 53)$ (table 5). The contractions to exogenous methacholine were not affected by magnesium, excluding the possibility that the high magnesium concentrations altered ASM physiology itself. These findings show that the EFS, like in rats, acts also in the other species on neurons and not directly on ASM.

Differences in stimulation conditions compared to other ex vivo EFS studies on lung tissue (183) can be due to species or tissue variability, different electrode geometry, and different ionic strength of the cell culture medium, which all influence the electric field. In the present study, different species were compared in an identical setup, including nearly identical tissue preparation. These identical conditions are the basis for the conclusion about the differences between species with respect to neurally mediated bronchoconstriction. 
Under the standard conditions $(F=50 \mathrm{~Hz}, B=1 \mathrm{~ms}, \mathrm{~A}=200 \mathrm{~mA}, \mathrm{TW}=2.5 \mathrm{~s})$ mice showed no significant response. However, EFS elicited contractions in murine tracheal preparations in previous studies $(11 ; 12 ; 93)$ and in line with that neurally-evoked bronchoconstriction was observed in murine PCLS at higher electric fields (figure 29). Higher electric fields were also required in studies by Braun and colleagues (31) as well as Larsen and colleagues (93), who used pulse durations of $2 \mathrm{~ms}$ when stimulating murine tracheal smooth muscle segments. In contrast, Bachar and colleagues $(11 ; 12)$ only used pulse durations of $0.8 \mathrm{~ms}$, but train widths lasted $60 \mathrm{~s}$ and were therefore much longer than the $2.5 \mathrm{~s}$ within this study. Hence, the observations made here are reasonable concluding that murine preparations are less susceptible to EFS and require harsher EFS conditions. Nonetheless, those harsher conditions seem still to neurons specifically, since magnesium blocked the EFS-induced airway response (figure 29 C). In ovine PCLS, on the other hand, airways were highly excitable (figures 28 and 30) and nerve activation was observed already at low frequencies or even after one single electrical stimulus $(0.4 \mathrm{~Hz}$ at train width $2.5 \mathrm{~s})$. Overall, the $\mathrm{EF}_{50}$ values observed in the present study are in line with the literature. The $\mathrm{EF}_{50}$ of $(0.5 \pm 0.2) \mathrm{Hz}$ is in the range of that for ovine bronchial rings and tracheal segments of about $0.2 \mathrm{~Hz}$ (156). The rat $\mathrm{EF}_{50}$ of $(16.5 \pm 0.8) \mathrm{Hz}$ was equal to that of $(16.7 \pm 4.9) \mathrm{Hz}$ in the previous part of the study and comparable to $(19.3 \pm 4.3) \mathrm{Hz}$ from isometric tension studies of rat tracheal smooth muscle (61). The guinea pig $\mathrm{EF}_{50}$ of $(7.0 \pm 1.2) \mathrm{Hz}$ is close to the $5.3 \mathrm{~Hz}$ and $3.7 \mathrm{~Hz}$ observed by Altiere and colleagues ( 8 ) when studying cholinergic responses in the trachea and tachykinergic responses in the bronchus, respectively. For human airways, the $\mathrm{EF}_{50}$ of $(8.1 \pm 0.8) \mathrm{Hz}$ herein is close to $(8.9 \pm 0.4) \mathrm{Hz}$ that was obtained in human bronchi before (47). Among all species studied here, the $\mathrm{EF}_{50}$ values of guinea pigs $((7.0 \pm 1.2) \mathrm{Hz})$ are closest to that of humans $((8.1 \pm 0.8) \mathrm{Hz})$, providing another argument for the conclusion that guinea pig airways are a reasonable proxy for human airways.

The differences between the species within this study are not explained by differences in ASM cells, because the responses to methacholine are very similar in all species $(68 ; 111 ; 145 ; 151 ; 155$; 188) and the experiments with magnesium als exclude direct electric activation of ASM. Possible explanations are differences in neurotransmitter release or neurotransmitter degrading enzymes such as the acetylcholine esterase that may vary in amount or activity $(86 ; 113 ; 148)$. However, one may speculate that the type of innervation, i.e., cholinergic, adrenergic, eNANC or iNANC, is the most relevant reason for the differences between species. For instance the repetitive reversible contraction in rat is cholinergic (152), whereas the steady contraction without relaxation in guinea pigs (figure 28) suggests a mechanism other than a cholinergic one, in which the neurotransmitter is not metabolized. 


\subsubsection{Pharmacological characterization of distal airway innervation}

The type of innervation was addressed by pharmacological agents: the muscarinic antagonist atropine (141) was used to show involvement of parasympathetic cholinergic nerves, whereas the TRPV1 channel activator capsaicin (126) or the unspecific TRP channel blockers SKF96365 and Ruthenium red (140) were used to demonstrate eNANC nerves. Relaxant nerve responses were addressed by the sympathetic adrenergic antagonist propranolol (82) or by the NO synthase inhibitor L-NAME to prevent NO release from iNANC nerves (95).

Atropine blocked the EFS-induced bronchoconstriction in PCLS from rats, guinea pigs, sheep, marmosets and humans (figures 31 and 33), indicating that these species receive cholinergic innervation in the distal part of their lungs. These findings extend former studies on larger airways $(8 ; 14 ; 54 ; 61 ; 70 ; 158)$ and they are important, because innervation is unevenly distributed along the tracheobronchial tree (8). This is also true for tachykinergic innervation in guinea pigs that so far was thought to supply predominantly the large airways (8). In this species distal airways seem to be supplied by both cholinergic and tachykinergic nerves, as shown by the effects of atropine and the TRP channel antagonists (figure 33). In contrast to the rats, in which cholinergic responses are weaker in smaller airways (152), distal airways from sheep or human were quite susceptible to EFS-induced cholinergic bronchoconstriction (figure 31), indicating relatively strong innervation of peripheral airways in these species. The residual airway contractions in the presence of atropine may be due to eNANC activation, or blockade of presynaptic muscarinic receptors by atropine, preventing the autoinhibitory effect of acetylcholine release $(14 ; 21)$ or due to release of SP that might potentiate the release of acetylcholine from cholinergic nerves (14).

Various types of TRP channels are expressed in the mammalian lung and are involved in the regulation of airway functions, in which particularly TRPV1 and TRPV4 play an important role. TRPV1 channels are generally expressed in non-myelinated C-fiber afferents, which innervate the airways and contain sensory neuropetides (79). Activation of TRPV1 cannels on nerves induces calcium influx into neurons, membrane depolarization and action potentials. Such excited chemosensitive nerves correspond to eNANC nerves, which induce bronchonconstriction by the release of SP or a related tachykinin (9). Therefore, the effects of capsaicin, a potent TRPV1 agonist (79; 126), were studied to estimate the role of eNANC innervation in the various species. Notably, only airways of guinea pigs and humans responded to capsaicin providing further evidence for the presence of tachykinergic nerves in the distal lung of these species (figure 32). Undem and coworkers (175) reported contractions of $(66 \pm 10) \%$ in guinea pig bronchi towards capsaicin and Spina with colleagues (158) made similar observations in human airways, albeit the effects were smaller here $(1 \mu \mathrm{M}:(2 \pm 3) \%, 10 \mu \mathrm{M}:(14 \pm 13) \%$ and with $100 \mu \mathrm{M}$ capsaicin $(44 \pm 40) \%$ of the maximal contraction by $10^{-4} \mathrm{M}$ methacholine). Also, findings by Lundberg and colleagues (100) support the notion that human airways are less responsive to capsaicin than those from guinea 
pigs, by showing that guinea pig tracheas are 1000-fold more sensitive to SP and 100-fold more sensitive to capsaicin than human bronchi. Further on, Lundberg's and colleagues' (100) study also supports the observation made in the present work that not all human airways are capsaicin sensitive. From studies with transmural EFS of human segmental bronchi, they report two different scenarios: one where contractions were abolished by atropine and another one where only tetrodotoxin (specific antagonist of voltage-gated sodium channels on nerve cells), but not atropine was effective. These findings suggest the existence of two types of human airways: either solely innervated by cholinergic nerves or innervated by both cholinergic and eNANC nerves.

That capsaicin failed to evoke clear bronchoconstriction in PCLS from marmosets is in line with data presented for marmoset trachea and bronchus, which weakly contracted by $(2.3 \pm 2.8) \%$ and $(8.0 \pm 6.6) \%$ of maximal contraction by $10^{-4} \mathrm{M}$ methacholine, respectively, after application of $100 \mu \mathrm{M}$ capsaicin (158). In conflict to capsaicin responses in ovine PCLS, sheep lambs exerted a reduction in tidal volume from $91 \mathrm{~mL}$ to $36 \mathrm{~mL}$ when capsaicin $(5 \mu \mathrm{g} / \mathrm{kg})$ was injected intravenously (10). This opposing finding may be explained either by regional differences along the tracheobronchial tree (trachea/bronchus vs. peripheral airways in PCLS) (8) or by reflex arcs in vivo (79), which are destroyed in the PCLS preparation. In rat and mouse bronchi, but probably also in marmoset and sheep airways, capsaicin may play a more prominent role in the activation of sensory nerves of the inhibitory system than in the excitatory system $(105 ; 164)$.

Nervous stimulation can also cause bronchorelaxation via either sympathetic or iNANC nerves (32; 143). In the present study, dilation of airways appeared only in PCLS from marmoset after multiple EFS (figures 28 and 34). The effects of propranolol and L-NAME demonstrate that this relaxant response was due to both sympathetic and iNANC innervation, because only the combination of the two inhibitors was effective in blocking the relaxant response. Although power analysis revealed the possibility of sympathetic contribution, iNANC contributions seem also to be involved for two reasons: Firstly, L-NAME prevented the return to baseline levels during EFS (figure $34 \mathrm{C}$, 2nd train) and secondly Spina and colleagues (158) reported relaxations in marmoset tracheae and bronchi at capsaicin concentrations as high as $100 \mu \mathrm{M}$ after the tissue was precontracted with methacholine. The marmoset maybe a useful model to study relaxant innervation of the lung, as interferencies with precontracting agents can be excluded. Whether the peripherial airways of the other species are innervated by relaxant nerves still has to be elucidated, because usually relaxation becomes apparent only in precontracted airways (43; 146).

In summary cholinergic innervations were found to contract airways of rats, guinea pigs, sheep, marmosets and humans. In addition eNANC nerves contracted guinea pig and human airways. Furthermore, the dilation of airways in marmoset PCLS was attributed to sympathetic and iNANC responses (table 6). 
Table 6: Peripheral airway innervation of distinct mammals as verified in the current examination

\begin{tabular}{|lcccc|}
\hline Species & Cholinergic & Adrenergic & eNANC & iNANC \\
\hline Mouse & & & - & \\
Rat & + & & - & \\
Guinea Pig & + & & + & \\
Sheep & + & + & - & + \\
Marmoset & + & & + & \\
Human & + & & & \\
\hline
\end{tabular}

eNANC, excitatory nonadrenergic noncholinergic; iNANC, inhibitory nonadrenergic noncholinergic; +, proven; -, insignificant.

\subsubsection{Phylogenetic considerations}

Airway pharmacology varies strikingly between species, not only with respect to neural transmission, but also with respect to various mediators and transmitters. For instance, airways from rodents, marmosets or rhesus monkeys do respond weakly or not at all to LTs $(67 ; 155)$, that readily contract airways in humans (37), cynomolgus, baboons (155) and guinea pigs (46; 66). All these studies suggest that, regarding airway pharmacology, guinea pigs resemble humans more closely than mice or rats do $(121 ; 161)$.

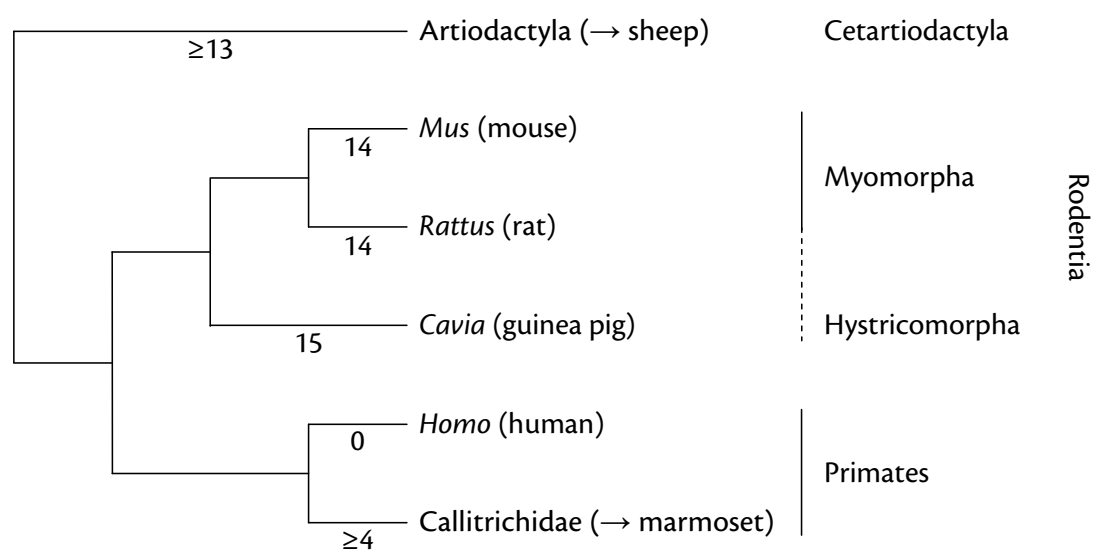

Figure 35: Phylogenetic relationships among the examined species. Relationships are deduced from Murphy and colleagues (122), who analysed 9779 base pairs from 15 nuclear and three mitochondrial DNA genes among 64 placental mammals and two marsupials. Except for the sheep all animal belong to one clade, the Neotropical order of Xenarthra. There is current debate on rodent monophyly or polyphyly and wether the guinea pig is a rodent or not (36). However, in line with D'Erchia's and colleagues' (36) phylogentic study on the whole mitchondrial genome, the Cavia separates earlier than Myomorpha and is traditionally classified as Hystricomorpha. The marmoset is considered as a non-human primate and belongs to the family of Callitrichidae, for which Murphy and colleagues (122) refer to the Callimico. Direct data for sheep also lacked and therefore the sheep was categorized to Artiodactyla. Numbers indicate the total count of branches beginning from human up to the examined species as suggested by Murphy and colleagues (122) for the interrelationship of their 66 studied Amniota, in which the minimum evolution topology was estimated by neighbour joining using maximum likelihood distances. 
The physiological observations might suggest a close phylogenetic relationship between guinea pigs and humans. Therefore, a phylogenetic tree based on Murphy's and colleagues' examinations (122) was drawn for all species within in this study (figure 35). Interestingly, however, this mapping does not indicate an exceptional close relationship between guinea pigs and humans, although this may be due to the selection of genes that were not specific for the lungs.

\subsection{Conclusion}

PCLS are widely used in airway pharmacology. In the present study EFS of PCLS was introduced as a novel way to study neural airway responses. The finding that in PCLS airways contract in response to electrical fields and that this response is sensitive to magnesium indicates that terminal nerves remain intact in this preparation and can be activated specifically. PCLS were prepared from different species including humans and peripheral neural airway responses were characterized. The present findings suggest that airways of rats, guinea pigs, sheep, marmosets and human do all receive cholinergic innervations. Moreover, distal airways from guinea pigs and humans also receive eNANC innervations, whereas marmoset airways display relaxations by sympathetic and iNANC nerves.

In the allergic rat model no AHR was observed, indicating that increased release of acetylcholine from postganglionic parasympathetic nerves does not occur and that acetylcholine receptors and the postreceptor transduction mechanisms are not affected by allergen exposure in rat.

Since the cholinergic response can be influenced pharmacologically in EFS of PCLS and log IC $\mathrm{C}_{50}$ for antagonists can be detrmined, this new method allows the screening of drugs affecting neurally evoked bronchoconstriction. As the guinea pig resembled the human response profile best, this species is particularly suitable for studies on the role of peripheral airway innervations and their involvement in human lung diseases. 


\section{Summary}

Chronic airway diseases, such as bronchial asthma and chronic obstructive pulmonary disease (COPD), are the fourth-leading cause of death in developed countries and have a high personal, societal, and economical impact. Recurring inflammation is one characteristic of those diseases leading to airway remodelling and modulating the function of nerves resulting in excessive bronchoconstriction. The precision-cut lung slice (PCLS) technique is widely used in pulmonary pharmacology, but neurally-induced bronchoconstriction has not been studied yet. Hence, the aim of this study was to develop a system, which specifically activates nerves within PCLS of different species, allowing the investigation of neural function and its pharmacological interference throughout the whole lung - also in an allergic animal model.

Electric field stimulation (EFS) is considered to activate nerve endings in tissue preparations. Therefore, a device was constructed in which the PCLS were faced by two platinum electrodes and which delivered serial rectangular electric stimuli. EFS-induced bronchoconstriction in PCLS was monitored by videomicroscopy.

EFS of PCLS was first established for the rat. EFS conditions were systematically studied and stable repeated airway contractions were obtained at a frequency of $50 \mathrm{~Hz}$, pulse duration of $1 \mathrm{~ms}$, current of $200 \mathrm{~mA}$ and train width of $2.5 \mathrm{~s}$. Magnesium antagonized airway contractions induced by EFS, but not by methacholine, indicating that they are neurally evoked. The EFS-triggered contractions were increased by the acetylcholine esterase inhibitor neostigmine and were highly reproducible, which allowed the study of muscarinic antagonists in consecutive EFS-trains. The decadic logarithmic inhibitory concentration of half-maximal effect $\left(\log I C_{50} M\right)$ of antagonists was determined in EFS of PCLS and revealed ipratropium (-10.0 \pm 0.4$)$ being more potent than atropine $(-8.8 \pm 0.4)$ and 4 -DAMP $(-8.4 \pm 0.3)$. Furthermore, it was tested if thromboxane facilitates cholinergic bronchoconstriction by using the thromboxane prostanoid receptor antagonist SQ29548, but no effect was observed. In EFS of PCLS on different airway sizes, larger airways showed stronger response than smaller ones.

In a model of allergic asthma, Brown-Norway rats were sensitized against ovalbumin (OVA). Besides haemorrhages in the lung also airway contractions in PCLS after OVA-provocation were found, pointing to a persisting inflammation and an acute allergic response, respectively. However, in PCLS from these rats no airway hyperresponsiveness was found in response to methacholine or EFS.

Since airway responses are known to exhibit considerable species differences, the neural responses of PCLS prepared from mice, guinea pigs, sheep, marmosets and humans were examined. Peripheral neurons were activated either by EFS or additionally, in order to probe excitatory nonadrenergic 
noncholinergic (NANC) nerves, by capsaicin. Bronchoconstriction in response to EFS varied between species in magnitude ( $(2 \pm 2) \%$ in mouse to $(61 \pm 26) \%$ in guinea pig) and sensitivity (half-maximal response in frequency-response curves: $(0.5 \pm 0.2) \mathrm{Hz}$ in sheep to $(23 \pm 7) \mathrm{Hz}$ in marmoset). As in rats, atropine antagonized the EFS-induced bronchoconstriction in PCLS from guinea pigs, sheep, marmosets and humans. Capsaicin caused bronchoconstriction only in human and guinea pig lungs. Transient receptor potential channel antagonists (SKF96365/Ruthenium red) inhibited airway contractions after EFS or capsaicin addition in guinea pigs. Only marmoset airways showed airway dilation after multiple EFS and this was blocked by propranolol and L-NAME.

In conclusion, the findings within this study show that nerve endings remain intact in PCLS. The present method is useful to study neurogenic responses in airways of different size and pharmacological interventions are possible. In rat PCLS, lesser sensitivity to EFS with smaller airways is in contrast to the response of exogenously applied methacholine, which suggests that parasympathetic bronchoconstriction in the airways seems to be balanced by inversely arranged innervation and acetylcholine-responsiveness: rich innervations with low acetylcholineresponsiveness in large airways opposed to more sparse innervations with high sensitivity in smaller airways. In the rat model of allergic asthma, increased release of acetylcholine from postganglionic parasympathetic nerves does not take place and acetylcholine receptors and the postreceptor transduction mechanisms are not affected after allergen sensitization. As in rats, airways of guinea pigs, sheep, marmosets and humans do all receive cholinergic innervations. Moreover, distal guinea pig and human airways also receive excitatory NANC innervations. Marmoset airways even display relaxations by sympathetic and inhibitory NANC nerves. As guinea pig resembled the human response profile best, this model may be particularly suited for studies on the role of peripheral airway innervation and its involvement in human lung diseases. 


\section{Deutsche Zusammenfassung}

Chronische Atemwegserkrankungen, wie Asthma bronchiale und die chronisch obstruktive Lungenerkrankung (COPD), sind die vierthäufigste Todesursache in Industrieländern und haben einen bedeutenden persönlichen, sozialen und ökonomischen Einfluss. Diesen Erkrankungen liegt eine chronische Entzündung zugrunde, die zum Umbau der Atemwege führt und die Nervenfunktion moduliert, so dass eine exzessive Bronchokonstriktion auftritt. Lebende Lungenschnitte (PCLS) werden in der Lungenpharmakologie zu unterschiedlichen Zwecken genutzt, aber neuronale Fragestellungen wurden in PCLS noch nicht untersucht. Das Ziel dieser Arbeit war es daher, ein System zu entwickeln, das spezifisch die Nerven in PCLS verschiedener Spezies aktiviert, um so die neuronale Funktion und ihre pharmakologische Beeinflussung in Atemwegen zu studieren. Ein Tiermodell des allergischen Asthmas sollte Aufschluss über eine etwaige Veränderung der Nervenfunktion in der Krankheit geben.

Elektrische Feldstimulation (EFS) wird genutzt, um Nervenendigungen in Gewebepräparationen zu aktivieren. In dieser Arbeit wurde die EFS an PCLS bewerkstelligt, indem PCLS zwischen zwei Platinelektroden platziert wurden und seriell elektrische Rechteckimpulse angelegt wurden. Die Bronchokonstriktion nach EFS wurde mit Hilfe der Videomikroskopie verfolgt.

Das ModellderEFSwurdezuerstan PCLSderRatteentwickelt.Die daselektrischeFeld bestimmenden Bedingungen wurden systematisch untersucht und reproduzierbare Atemwegskontraktionen wurden bei einer Frequenz von $50 \mathrm{~Hz}$, einer Reizbreite von 1 ms, einem Strom von 200 mA und einer Seriendauer von 2,5 s gefunden. Die EFS-induzierte Bronchokonstriktion ist neuronal bedingt, da Magnesium die Bronchokonstriktion in der EFS antagonisierte, aber nicht die durch Methacholin hervorgerufene Kontraktion der glatten Muskulatur der Atemwege. Die durch EFS ausgelösten Kontraktionen wurden durch den Acteylcholinesteraseinhibitor Neostigmin verstärkt und waren höchst reproduzierbar, sodass muskarinische Rezeptorantagonisten in aufeinanderfolgenden EFSSerien untersucht werden konnten. Darin wurde die logarithmische inhibitorische Konzentration des halbmaximalen Effekts ( $\left.\log I C_{50} M\right)$ ermittelt und es zeigte sich, dass Ipratropium (-10.0 \pm 0.4$)$ potenter als Atropin (-8.8 \pm 0.4$)$ und 4-DAMP $(-8.4 \pm 0.3)$ wirkte. In einem anderen Ansatz wurde geprüft, ob Thromboxan die parasympathisch-cholinerge Bronchokonstriktion begünstigt, indem SQ29548 als Thromboxanprostanoid-Rezeptor-Antagonist eingesetzt wurde. Hierbei konnte jedoch kein Effekt festgestellt werden. In der EFS an PCLS mit unterschiedlicher Atemwegsgröße zeigte sich, dass größere Atemwege reaktiver waren als Kleinere.

Als Tiermodell des allergischen Asthmas dienten gegen Ovalbumin sensibilisierte Brown-Norway Ratten. Hämorrhagien in der Lunge und Atemwegskontraktion in PCLS nach Allergenprovokation deuteten auf eine bestehende Entzündung und eine akute allergische Reaktion hin. Allerdings trat Atemwegshyperreaktion weder nach Methacholinzugabe noch nach EFS auf. 
Neben der Ratte wurde die neuronale Reaktion in PCLS von Maus, Meerschwein, Schaf, Marmoset und Mensch untersucht, da die Innervation der Atemwegsmuskulatur in verschiedenen Spezies sehr unterschiedlich ausfällt. Periphere Neuronen wurden per EFS oder zusätzlich durch Capsaicin als Stimulanz exzitatorischer nicht-adrenerger nicht-cholinerger (NANC) Nerven aktiviert. Die durch EFS hervorgerufene Bronchokonstriktion variierte zwischen den Spezies im Ausmaß $((2 \pm 2) \%$ in der Maus bis $(61 \pm 26) \%$ im Meerschwein) und in der Sensitivität (halbmaximale Antwort in der Frequenz-Wirkungskurve: $(0,5 \pm 0,2) \mathrm{Hz}$ im Schaf bis $(23 \pm 7) \mathrm{Hz}$ im Marmoset). Wie bei der Ratte antagonisierte Atropin die EFS induzierte Bronchokonstriktion in PCLS des Meerschweins, Schafs, Marmosets und Menschen. Capsaicin bewirkte eine Bronchokonstriktion nur in humanen PCLS sowie in denen des Meerschweins. Ruthenium Red und SKF96365 sind Inhibitoren an TRP-Kanälen (engl. transient receptor potential channels) und antagonisierten die Atemwegskontraktion im Meerschwein sowohl nach EFS als auch nach Zugabe von Capsaicin. Lediglich Marmoset-Atemwege zeigten eine Relaxation in der EFS an PCLS, die sensitiv gegenüber Propranolol und L-NAME war.

Zusammenfassend konnte gezeigt werden, dass Nervenendigungen in PCLS intakt bleiben. Die entwickelte Methode eignet sich dazu neurogene Mechanismen in Abhängigkeit der Atemwegsgröße zu untersuchen und pharmakologische Beeinflussungen der EFS-induzierten Antwort sind möglich. Die geringere Antwort mit kleineren Atemwegen in der EFS widerspricht der höheren Anfälligkeit kleiner Atemwege nach äußerer Zugabe von Methacholin. Dies deutet jedoch auf eine geschickte Regulation der parasympathischen Bronchokonstriktion hin: Größere Atemwege scheinen stärker innerviert als Kleine, sind aber dafür weniger anfällig gegenüber Acetylcholin als die Kleinen. Im Rattenmodell des allergischen Asthmas konnte gezeigt werden, dass die cholinerge Antwort unverändert ist. Entsprechend der Ratte weisen die Spezies Meerschwein, Schaf, Marmoset und Mensch alle cholinerge Innervierung auf. Zudem sind distale Atemwege des Meerschweins und Menschen noch exzitatorisch NANC innerviert, wohingegen die Dilation der Atemwege des Marmosets durch adrenerge und inhibitorsiche NANC Nerven bestimmt ist. Meerschwein PCLS wiesen die größten Gemeinsamkeiten mit humanen PCLS auf, und dürften sich deshalb in Zukunft besonders für klinisch relevante Untersuchungen neuronaler Atemwegsreaktionen eignen. 


\section{References}

1. Abraham WM. Animal models of asthma. In: Asthma \& Rhinitis, edited by Busse WW and Holgate ST. Eds. Oxford: Blackwell Science, 1205-1227, 2000.

2. Abraham WM. Modeling of asthma, COPD and cystic fibrosis in sheep. Pulm Pharmacol Ther 21: 743-754, 2008.

3. Aizawa $\mathbf{H}$ and Hirose $\mathbf{T}$. A possible mechanism of airway hyperresponsiveness induced by prostaglandin F2 alpha and thromboxane A2. Prostaglandins Leukot Essent Fatty Acids 33: 185-189, 1988.

4. Aizawa H, Takata S, Shigyo M, Matsumoto K, Koto H, Inoue $H$ and Hara N. Effect of BAY u3405, a thromboxane A2 receptor antagonist, on neuro-effector transmission in canine tracheal tissue. Prostaglandins Leukot Essent Fatty Acids 53: 213-217, 1995.

5. Aktories K, Förstermann U, Hofmann FB and Starke K. Allgemeine und spezielle Pharmakologie und Toxikologie. Urban \& Fischer Verlag/Elsevier, 2004.

6. Alexander SPH. 7TM-Receptors. Br J Pharmacol 158: S5-S101, 2009.

7. Allen IC, Hartney JM, Coffman TM, Penn RB, Wess J and Koller BH. Thromboxane A2 induces airway constriction through an M3 muscarinic acetylcholine receptor-dependent mechanism. Am J Physiol Lung Cell Mol Physiol 290: L526-L533, 2006.

8. Altiere RJ, Diamond L and Thompson DC. Omega-conotoxin-sensitive calcium channels modulate autonomic neurotransmission in guinea pig airways. J Pharmacol Exp Ther 260: 98-103, 1992.

9. Andersson RG and Grundstrom N. Innervation of airway smooth muscle. Efferent mechanisms. Pharmacol Ther 32: 107-130, 1987.

10. Arsenault J, Moreau-Bussiere F, Reix P, Niyonsenga T and Praud JP. Postnatal maturation of vagal respiratory reflexes in preterm and full-term lambs. J Appl Physiol 94: 1978-1986, 2003.

11. Bachar O, Adner M, Uddman R and Cardell LO. Nerve growth factor enhances cholinergic innervation and contractile response to electric field stimulation in a murine in vitro model of chronic asthma. Clin Exp Allergy 34: 1137-1145, 2004. 
12. Bachar O, Adner M, Uddman R and Cardell LO. Prolonged exposure to NT-3 attenuates cholinergic nerve-mediated contractions in cultured murine airways. Respir Physiol Neurobiol 147: 81-89, 2005.

13. Bara I, Ozier A, Tunon de Lara JM, Marthan R and Berger P. Pathophysiology of bronchial smooth muscle remodelling in asthma. Eur Respir J 36: 1174-1184, 2010.

14. Barnes PJ. Cholinergic control of airway smooth muscle. Am Rev Respir Dis 136: S42-S45, 1987.

15. Barnes PJ. New concepts in the pathogenesis of bronchial hyperresponsiveness and asthma. J Allergy Clin Immunol 83: 1013-1026, 1989.

16. Barnes PJ. Neural mechanisms in asthma. Br Med Bull 48: 149-168, 1992.

17. Barnes PJ. Immunology of asthma and chronic obstructive pulmonary disease. Nat Rev Immunol 8: 183-192, 2008.

18. Barnes PJ. Mechanisms in COPD compared with asthma. Breathe 5: 134-144, 2008.

19. Barnes PJ, Nadel JA, Skoogh BE and Roberts JM. Characterization of beta adrenoceptor subtypes in canine airway smooth muscle by radioligand binding and physiological responses. J Pharmacol Exp Ther 225: 456-461, 1983.

20. Beavo JA and Brunton LL. Cyclic nucleotide research - still expanding after half a century. Nat Rev Mol Cell Biol 3: 710-718, 2002.

21. Belmonte KE. Cholinergic pathways in the lungs and anticholinergic therapy for chronic obstructive pulmonary disease. Proc Am Thorac Soc 2: 297-304, 2005.

22. Belvisi MG, Ward JK, Mitchell JA and Barnes PJ. Nitric oxide as a neurotransmitter in human airways. Arch Int Pharmacodyn Ther 329: 97-110, 1995.

23. Bischof RJ, Snibson K, Shaw R and Meeusen EN. Induction of allergic inflammation in the lungs of sensitized sheep after local challenge with house dust mite. Clin Exp Allergy 33: 367375, 2003.

24. Bischof RJ, Snibson KJ, Velden JV and Meeusen EN. Immune response to allergens in sheep sensitized to house dust mite. J Inflamm 5: 16-26, 2008. 
25. Bosse Y, Chin LY, Pare PD and Seow CY. Adaptation of airway smooth muscle to basal tone: relevance to airway hyperresponsiveness. Am J Respir Cell Mol Biol 40: 13-18, 2009.

26. Bosse Y, Chin LY, Pare PD and Seow CY. Chronic activation in shortened airway smooth muscle: a synergistic combination underlying airway hyperresponsiveness? Am J Respir Cell Mol Biol 42: 341-348, 2010.

27. Botelho FM, Llop-Guevara A, Trimble NJ, Nikota JK, Bauer CM, Lambert KN, Kianpour S, Jordana $\mathbf{M}$ and Stampfli MR. Cigarette smoke differentially impacts eosinophilia and remodeling in a rouse dust mite asthma model. Am J Respir Cell Mol Biol, in press, 2011.

28. Bradley SL and Russell JA. Distribution of histamine receptors in isolated canine airways. J Appl Physiol 54: 693-700, 1983.

29. Bramley AM, Roberts CR and Schellenberg RR. Collagenase increases shortening of human bronchial smooth muscle in vitro. Am J Respir Crit Care Med 152: 1513-1517, 1995.

30. Braun A, Appel E, Baruch R, Herz U, Botchkarev V, Paus R, Brodie C and Renz H. Role of nerve growth factor in a mouse model of allergic airway inflammation and asthma. Eur J Immunol 28: 3240-3251, 1998.

31. Braun A, Lommatzsch M, Neuhaus-Steinmetz U, Quarcoo D, Glaab T, McGregor GP, Fischer A and Renz H. Brain-derived neurotrophic factor (BDNF) contributes to neuronal dysfunction in a model of allergic airway inflammation. Br J Pharmacol 141: 431-440, 2004.

32. Canning BJ and Fischer A. Neural regulation of airway smooth muscle tone. Respir Physiol 125: 113-127, 2001.

33. Carstairs JR and Barnes PJ. Visualization of vasoactive intestinal peptide receptors in human and guinea pig lung. J Pharmacol Exp Ther 239: 249-255, 1986.

34. Chew AD, Hirota JA, Ellis R, Wattie J, Inman MD and Janssen LJ. Effects of allergen on airway narrowing dynamics as assessed by lung-slice technique. Eur Respir J 31: 532-538, 2008.

35. Cowl CT. Occupational asthma: review of assessment, treatment, and compensation. Chest 139: 674-681, 2011.

36. D'Erchia AM, Gissi C, Pesole G, Saccone C and Arnason U. The guinea-pig is not a rodent. Nature 381: 597-600, 1996. 
37. Dahlen SE, Hedqvist P, Hammarstrom $\mathbf{S}$ and Samuelsson B. Leukotrienes are potent constrictors of human bronchi. Nature 288: 484-486, 1980.

38. Dassow C. Influence of chemical and mechanical stress on precision-cut lung slices (Dissertation). RWTH Aachen University, 2010.

39. Dassow C, Wiechert L, Martin C, Schumann S, Muller-Newen G, Pack O, Guttmann J, Wall WA and Uhlig S. Biaxial distension of precision-cut lung slices. J Appl Physiol 108: 713 721, 2010.

40. del Castillo J and Engbaek L. The nature of the neuromuscular block produced by magnesium. J Physiol 124: 370-384, 1954.

41. Delmotte $\mathbf{P}$ and Sanderson $\mathbf{M J}$. Ciliary beat frequency is maintained at a maximal rate in the small airways of mouse lung slices. Am J Respir Cell Mol Biol 35: 110-117, 2006.

42. Devillier P, Acker GM, Advenier C, Marsac J, Regoli D and Frossard N. Activation of an epithelial neurokinin NK-1 receptor induces relaxation of rat trachea through release of prostaglandin E2.J Pharmacol Exp Ther 263: 767-772, 1992.

43. Doidge JM and Satchell DG. Adrenergic and non-adrenergic inhibitory nerves in mammalian airways. J Auton Nerv Syst 5: 83-99, 1982.

44. Dong JM, Leung T, Manser E and Lim L. CAMP-induced morphological changes are counteracted by the activated RhoA small GTPase and the Rho kinase ROKalpha. J Biol Chem 273: 22554-22562, 1998.

45. Döring HJ, Classen HG and Dehnert H. Muscle contractions - isotonic, auxotonic, isometric - Electronic measurement and analogue calculation and evaluation. In: HSE Biological Measurement Techniques, Biomesstechnik-Verlag March, 26-61, 1986.

46. Drazen JM, Austen KF, Lewis RA, Clark DA, Goto G, Marfat A and Corey EJ. Comparative airway and vascular activities of leukotrienes $C-1$ and $D$ in vivo and in vitro. Proc Natl Acad Sci U S A 77: 4354-4358, 1980.

47. Dupont LJ, Pype JL, Demedts MG, De LP, Deneffe G and Verleden GM. The effects of 5-HT on cholinergic contraction in human airways in vitro. Eur Respir J 14: 642-649, 1999. 
48. Ebina M, Yaegashi H, Chiba R, Takahashi T, Motomiya M and Tanemura M. Hyperreactive site in the airway tree of asthmatic patients revealed by thickening of bronchial muscles. $A$ morphometric study. Am Rev Respir Dis 141: 1327-1332, 1990.

49. Eltze M. Tracheal Preparations. In: Methods in Pulmonary Research, edited by Uhlig S and Taylor AE. Birkhäuser, 71-90, 1998.

50. Elwood W, Sakamoto T, Barnes PJ and Chung KF. Allergen-induced airway hyperresponsiveness in Brown-Norway rat: role of parasympathetic mechanisms. J Appl Physiol 75: 279-284, 1993.

51. Faller $\mathbf{A}$ and Schünke $\mathbf{M}$. Der Körper des Menschen - Einführung in Bau und Funktion. Georg Thieme Verlag, 2008.

52. Fedan JS, Van Scott MR and Johnston RA. Pharmacological techniques for the in vitro study of airways. J Pharmacol Toxicol Methods 45: 159-174, 2001.

53. Feldman S and Karalliedde L. Drug interactions with neuromuscular blockers. Drug Saf 15: 261-273, 1996.

54. Fernandes LB, Henry PJ and Goldie RG. Endothelin-1 potentiates cholinergic nervemediated contraction in human isolated bronchus. Eur Respir J 14: 439-442, 1999.

55. Frossard $\mathbf{N}$ and Barnes J. Effect of tachykinins in small human airways. Neuropeptides 19: 157-161, 1991.

56. Frossard $\mathbf{N}$ and Muller F. Epithelial modulation of tracheal smooth muscle response to antigenic stimulation. J Appl Physiol 61: 1449-1456, 1986.

57. Fujisawa K, Onoue H, Abe K and Ito Y. Multiple calcium channels regulate neurotransmitter release from vagus nerve terminals in the cat bronchiole. Br J Pharmacol 128: 262-268, 1999.

58. Garcia JG, Davis HW and Patterson CE. Regulation of endothelial cell gap formation and barrier dysfunction: role of myosin light chain phosphorylation. J Cell Physiol 163: 510-522, 1995.

59. Gillissen A, Schafer H, Tasci S and Ewig S. From physiopathology to new developments in pharmacotherapy of bronchial asthma - a look into the future. Pneumologie 54: 345-354, 2000. 
60. Glaab T, Hoymann HG, Hohlfeld JM, Korolewitz R, Hecht M, Alarie Y, Tschernig T, Braun A, Krug $\mathbf{N}$ and Fabel $\mathbf{H}$. Noninvasive measurement of midexpiratory flow indicates bronchoconstriction in allergic rats. J Appl Physiol 93: 1208-1214, 2002.

61. Gonzalez $\mathbf{O}$ and Santacana GE. Effect of low temperature on tracheal smooth muscle contractile and relaxing responses evoked by electrical field stimulation. P R Health Sci J 20: 237-244, 2001.

62. Gosens R, Zaagsma J, Meurs $\mathbf{H}$ and Halayko AJ. Muscarinic receptor signaling in the pathophysiology of asthma and COPD. Respir Res 7: 73, 2006.

63. Gourlet P, Vandermeers A, Vertongen P, Rathe J, de NP, Cnudde J, Waelbroeck M and Robberecht P. Development of high affinity selective VIP1 receptor agonists. Peptides 18: 1539-1545, 1997.

64. Handoyo S and Rosenwasser LJ. Asthma phenotypes. Curr Allergy Asthma Rep 9: 439-445, 2009.

65. Hazari MS, Pan JH and Myers AC. Nerve growth factor acutely potentiates synaptic transmission in vitro and induces dendritic growth in vivo on adult neurons in airway parasympathetic ganglia. Am J Physiol Lung Cell Mol Physiol 292: L992-1001, 2007.

66. Hedqvist P, Dahlen SE, Gustafsson L, Hammarstrom S and Samuelsson B. Biological profile of leukotrienes C4 and D4. Acta Physiol Scand 110: 331-333, 1980.

67. Held HD, Martin C and Uhlig S. Characterization of airway and vascular responses in murine lungs. BrJ Pharmacol 126: 1191-1199, 1999.

68. Henjakovic M, Martin C, Hoymann HG, Sewald K, Ressmeyer AR, Dassow C, Pohlmann G, Krug N, Uhlig S and Braun A. Ex vivo lung function measurements in precision-cut lung slices (PCLS) from chemical allergen-sensitized mice represent a suitable alternative to in vivo studies. Toxicol Sci 106: 444-453, 2008.

69. Henjakovic M, Sewald K, Switalla S, Kaiser D, Muller M, Veres TZ, Martin C, Uhlig S, Krug $\mathbf{N}$ and Braun $\mathbf{A}$. Ex vivo testing of immune responses in precision-cut lung slices. Toxicol Appl Pharmacol 231: 68-76, 2008. 
70. Henry PJ, Shen A, Mitchelson F and Goldie RG. Inhibition by endothelin-1 of cholinergic nerve-mediated acetylcholine release and contraction in sheep isolated trachea. $\mathrm{Br} J$ Pharmacol 118: 762-768, 1996.

71. Herz U, Braun A, Ruckert R and Renz H. Various immunological phenotypes are associated with increased airway responsiveness. Clin Exp Allergy 28: 625-634, 1998.

72. Hirota S, Helli $\mathbf{P}$ and Janssen LJ. lonic mechanisms and Ca2+ handling in airway smooth muscle. Eur Respir J 30: 114-133, 2007.

73. Hirshman CA, Malley A and Downes H. Basenji-Greyhound dog model of asthma: reactivity to Ascaris suum, citric acid, and methacholine. J Appl Physiol 49: 953-957, 1980.

74. Hunter DD, Myers AC and Undem BJ. Nerve growth factor-induced phenotypic switch in guinea pig airway sensory neurons. Am J Respir Crit Care Med 161: 1985-1990, 2000.

75. Hylkema MN, Hoekstra MO, Luinge $\mathbf{M}$ and Timens $\mathbf{W}$. The strength of the OVA-induced airway inflammation in rats is strain dependent. Clin Exp Immunol 129: 390-396, 2002.

76. Jacoby DB, Gleich GJ and Fryer AD. Human eosinophil major basic protein is an endogenous allosteric antagonist at the inhibitory muscarinic M2 receptor. J Clin Invest 91: 1314-1318, 1993.

77. James A and Carroll N. Airway smooth muscle in health and disease; methods of measurement and relation to function. Eur Respir J 15: 782-789, 2000.

78. Jeremy JY, Mikhailidis DP and Dandona P. Muscarinic stimulation of prostanoid synthesis by the isolated rat trachea: calcium dependency and effect of cortisol and cigarette smoke. Eur J Pharmacol 160: 107-115, 1989.

79. Jia Y and Lee LY. Role of TRPV receptors in respiratory diseases. Biochim Biophys Acta 1772: 915-927, 2007.

80. Johri AM and Janssen LJ. N-Type $\mathrm{Ca}(2+)$ channels trigger release of excitatory and inhibitory neurotransmitter from nerve endings in canine bronchi. J Pharmacol Exp Ther 290: 847-853, 1999.

81. Joos GF, Lefebvre RA, Kips JC and Pauwels RA. Tachykinins contract trachea from Fischer 344 rats by interaction with a tachykinin NK1 receptor. Eur J Pharmacol 271: 47-54, 1994. 
82. Kamikawa $Y$ and Shimo $Y$. Inhibitory effects of sympathomimetic drugs on cholinergically mediated contractions of guinea-pig isolated tracheal muscle. J Pharm Pharmacol 38: 742747, 1986.

83. Kirschvink $\mathbf{N}$ and Reinhold P. Use of alternative animals as asthma models. Curr Drug Targets 9: 470-484, 2008.

84. Klinke R, Pape HC and Silbernagl S. Lehrbuch der Physiologie. Georg Thieme Verlag, 2005.

85. Knox AJ and Tattersfield AE. Airway smooth muscle relaxation. Thorax 50: 894-901, 1995.

86. KrejciE,Martinez-PenayValenzuela I,AmezianeRand AkaabouneM.Acetylcholinesterase dynamics at the neuromuscular junction of live animals. J Biol Chem 281: 10347-10354, 2006.

87. Kucharewicz I, Bodzenta-Lukaszyk A and Buczko W. Experimental asthma in rats. Pharmacol Rep 60: 783-788, 2008.

88. Kucharewicz I, Kasacka I, Pawlak D, Tankiewicz-Kwedlo A, Mroczko B, Buczko W and Bodzenta-Lukaszyk A. The concentration of kynurenine in rat model of asthma. Folia Histochem Cytobiol 46: 199-203, 2008.

89. Laberge S, Rabb H, Issekutz TB and Martin JG. Role of VLA-4 and LFA-1 in allergen-induced airway hyperresponsiveness and lung inflammation in the rat. Am J Respir Crit Care Med 151: 822-829, 1995.

90. Lambert RK, Codd SL, Alley MR and Pack RJ. Physical determinants of bronchial mucosal folding. J Appl Physiol 77: 1206-1216, 1994.

91. Lancas T, Kasahara DI, Prado CM, Tiberio IF, Martins MA and Dolhnikoff M. Comparison of early and late responses to antigen of sensitized guinea pig parenchymal lung strips. J Appl Physiol 100: 1610-1616, 2006.

92. Lang P, Gesbert F, espine-Carmagnat $M$, Stancou R, Pouchelet $M$ and Bertoglio J. Protein kinase $A$ phosphorylation of RhoA mediates the morphological and functional effects of cyclic AMP in cytotoxic lymphocytes. EMBO J 15: 510-519, 1996.

93. Larsen GL, Renz H, Loader JE, Bradley KL and Gelfand EW. Airway response to electrical field stimulation in sensitized inbred mice. Passive transfer of increased responsiveness with peribronchial lymph nodes. J Clin Invest 89: 747-752, 1992. 
94. Le Cras TD, Acciani TH, Mushaben EM, Kramer EL, Pastura PA, Hardie WD, Korfhagen TR, Sivaprasad U, Ericksen M, Gibson AM, Holtzman MJ, Whitsett JA and Hershey GK. Epithelial EGF receptor signaling mediates airway hyperreactivity and remodeling in a mouse model of chronic asthma. Am J Physiol Lung Cell Mol Physiol 300: L414-L421, 2011.

95. Li CG and Rand MJ. Evidence that part of the NANC relaxant response of guinea-pig trachea to electrical field stimulation is mediated by nitric oxide. Br J Pharmacol 102: 91-94, 1991.

96. Li W and Stephens NL. Auxotonic loading and airway smooth muscle shortening. Can J Physiol Pharmacol 72: 1458-1463, 1994.

97. Lieu T, Kollarik $\mathbf{M}$, Myers AC and Undem BJ. Neurotrophin and GDNF family ligand receptor expression in vagal sensory nerve subtypes innervating the adult guinea pig respiratory tract. Am J Physiol Lung Cell Mol Physiol 300: L790-L798, 2011.

98. Lüllmann H, Mohr K and Hein L. Pharmakologie und Toxikologie - Arzneimittelwirkungen verstehen - Medikamente gezielt einsetzen. Georg Thieme Verlag, 2003.

99. Lundberg JM. Tachykinins, sensory nerves, and asthma--an overview. Can J Physiol Pharmacol 73: 908-914, 1995.

100. Lundberg JM, Martling CR and Saria A. Substance P and capsaicin-induced contraction of human bronchi. Acta Physiol Scand 119: 49-53, 1983.

101. Maarsingh H, Bossenga BE, Bos IS, Volders HH, Zaagsma J and Meurs H. L-arginine deficiency causes airway hyperresponsiveness after the late asthmatic reaction. Eur Respir J 34: 191-199, 2009.

102. Maarsingh H, Leusink J, Bos IS, Zaagsma J and Meurs H. Arginase strongly impairs neuronal nitric oxide-mediated airway smooth muscle relaxation in allergic asthma. Respir Res 7: 6, 2006.

103. Maarsingh H, Tio MA, Zaagsma J and Meurs H. Arginase attenuates inhibitory nonadrenergic noncholinergic nerve-induced nitric oxide generation and airway smooth muscle relaxation. Respir Res 6: 23, 2005.

104. Maize DF, Fedan JS and Dey RD. Characterization of neural control and contractile function in airway smooth muscle of the ferret. Pulm Pharmacol Ther 11: 57-64, 1998. 
105. Manzini S. Bronchodilatation by tachykinins and capsaicin in the mouse main bronchus. $\mathrm{Br}$ J Pharmacol 105: 968-972, 1992.

106. Martin C. Study of pharmacological and inflammatory responses in precision cut lung slices. Stähle, 1997.

107. Martin C, Goggel R, Dal P, V, Vergelli C, Giovannoni P, Ernst M and Uhlig S. Airway relaxant and anti-inflammatory properties of a PDE4 inhibitor with low affinity for the highaffinity rolipram binding site. Naunyn Schmiedebergs Arch Pharmacol 365: 284-289, 2002.

108. Martin C, Held HD and Uhlig S. Differential effects of the mixed ET(A)/ET(B)-receptor antagonist bosentan on endothelin-induced bronchoconstriction, vasoconstriction and prostacyclin release. Naunyn Schmiedebergs Arch Pharmacol 362: 128-136, 2000.

109. Martin C, Ressmeyer AR, Dassow C, Sewald K, Hoymann HG, Braun A and Uhlig S. Comparison of asthmatic airway responses in rats in vivo and in vitro. Am J Respir Crit Care Med 179: A6037, 2009.

110. Martin C and Uhlig S. The method of precision-cut lung slices. In: Replacing animal models: a practical guide to creating and using biomimetic alternatives, Wiley-Blackwell, 2011.

111. Martin C, Uhlig S and Ullrich V. Videomicroscopy of methacholine-induced contraction of individual airways in precision-cut lung slices. Eur Respir J 9: 2479-2487, 1996.

112. Martin C, Ullrich V and Uhlig S. Effects of the thromboxane receptor agonist U46619 and endothelin-1 on large and small airways. Eur Respir J 16: 316-323, 2000.

113. Martinez-Pena y Valenzuela I and Akaaboune $\mathbf{M}$. Acetylcholinesterase Mobility and Stability at the Neuromuscular Junction of Living Mice. Mol Biol Cell 18: 2904-2911, 2007.

114. McCaig DJ. Autonomic responses of the isolated, innervated trachea of the guinea-pig: interaction with autonomic drugs, histamine and 5-hydroxytryptamine. Br J Pharmacol 88: 239-248, 1986.

115. McCarron JG, Olson ML, Rainbow RD, MacMillan D and Chalmers S. Ins $(1,4,5) \mathrm{P}(3)$ receptor regulation during 'quantal' $\mathrm{Ca}(2+)$ release in smooth muscle. Trends Pharmacol Sci 28: 271-279, 2007. 
116. Meurs H, Gosens R and Zaagsma J. Airway hyperresponsiveness in asthma: lessons from in vitro model systems and animal models. Eur Respir J 32: 487-502, 2008.

117. Meurs H, Santing RE, Remie R, van der Mark TW, Westerhof FJ, Zuidhof AB, Bos IS and Zaagsma J. A guinea pig model of acute and chronic asthma using permanently instrumented and unrestrained animals. Nat Protoc 1: 840-847, 2006.

118. Misawa M and Chiba Y. Repeated antigenic challenge-induced airway hyperresponsiveness and airway inflammation in actively sensitized rats. Jpn J Pharmacol 61: 41-50, 1993.

119. Mitchell RA, Herbert DA, Baker DG and Basbaum CB. In vivo activity of tracheal parasympathetic ganglion cells innervating tracheal smooth muscle. Brain Res 437: 157-160, 1987.

120. Moreno RH, Hogg JC and Pare PD. Mechanics of airway narrowing. Am Rev Respir Dis 133: 1171-1180, 1986.

121. Muccitelli RM, Tucker SS, Hay DW, Torphy TJ and Wasserman MA. Is the guinea pig trachea a good in vitro model of human large and central airways? Comparison on leukotriene-, methacholine-, histamine- and antigen-induced contractions. J Pharmacol Exp Ther 243: 467-473, 1987.

122. Murphy WJ, Eizirik E, Johnson WE, Zhang YP, Ryder OA and O'Brien SJ. Molecular phylogenetics and the origins of placental mammals. Nature 409: 614-618, 2001.

123. Nabel EG. Morbidity \& Mortality: 2007 Chart book on cardiovascular, lung, and blood diseases. National Institutes of Health, 2007.

124. Nadel JA, Cabezas GA and Austin JH. In vivo roentgenographic examination of parasympathetic innervation of small airways. Use of powdered tantalum and a fine focal spot x-ray tube. Invest Radiol 6: 9-17, 1971.

125. Naline E, Trifilieff A, Fairhurst RA, Advenier $\mathbf{C}$ and Molimard $\mathbf{M}$. Effect of indacaterol, a novel long-acting beta2-agonist, on isolated human bronchi. Eur Respir J 29: 575-581, 2007.

126. Nassenstein C, Kwong K, Taylor-Clark T, Kollarik M, Macglashan DM, Braun A and Undem BJ. Expression and function of the ion channel TRPA1 in vagal afferent nerves innervating mouse lungs. J Physiol 586: 1595-1604, 2008. 
127. Nassimi M, Schleh C, Lauenstein HD, Hussein R, Hoymann HG, Koch W, Pohlmann G, Krug N, Sewald K, Rittinghausen S, Braun A and Muller-Goymann C. A toxicological evaluation of inhaled solid lipid nanoparticles used as a potential drug delivery system for the lung. Eur J Pharm Biopharm 75: 107-116, 2010.

128. Nassimi M, Schleh C, Lauenstein HD, Hussein R, Lubbers K, Pohlmann G, Switalla S, Sewald K, Muller M, Krug N, Muller-Goymann CC and Braun A. Low cytotoxicity of solid lipid nanoparticles in in vitro and ex vivo lung models. Inhal Toxicol 21 Suppl 1: 104-109, 2009.

129. Netter FH. Farbatlanten der Medizin - Atmungsorgane. Georg Thieme Verlag, 1982.

130. Nie Z, Jacoby DB and Fryer AD. Etanercept prevents airway hyperresponsiveness by protecting neuronal $\mathrm{M} 2$ muscarinic receptors in antigen-challenged guinea pigs. $\mathrm{Br} J$ Pharmacol 156: 201-210, 2009.

131. Nie Z, Scott GD, Weis PD, Itakura A, Fryer AD and Jacoby DB. Role of TNF-alpha in virusinduced airway hyperresponsiveness and neuronal $M(2)$ muscarinic receptor dysfunction. $B r$ J Pharmacol, in press, 2011.

132. Nimmo AJ, Whitaker EM, Carstairs JR and Morrison JF. The autoradiographic localization of calcitonin gene-related peptide and substance $P$ receptors in human fallopian tube. Q J Exp Physiol 74: 955-958, 1989.

133. Noritake S, Ogawa K, Suzuki G, Ozawa K and Ikeda T. Pulmonary inflammation in brown Norway rats: possible association of environmental particles in the animal room environment. Exp Anim 56: 319-327, 2007.

134. Olin JL. Tiotropium: an inhaled anticholinergic for chronic obstructive pulmonary disease. Am J Health Syst Pharm 62: 1263-1269, 2005.

135. Osborne ML, Evans TW, Sommerhoff CP, Chung KF, Hirshman CA, Boushey HA and Nadel JA. Hypotonic and isotonic aerosols increase bronchial reactivity in basenji-greyhound dogs. Am Rev Respir Dis 135: 345-349, 1987.

136. Palmans E, Kips JC and Pauwels RA. Prolonged allergen exposure induces structural airway changes in sensitized rats. Am J Respir Crit Care Med 161: 627-635, 2000. 
137. Palmer JB, Cuss FM and Barnes PJ. VIP and PHM and their role in nonadrenergic inhibitory responses in isolated human airways. J Appl Physiol 61: 1322-1328, 1986.

138. Pan J, Rhode HK, Undem BJ and Myers AC. Neurotransmitters in airway parasympathetic neurons altered by neurotrophin-3 and repeated allergen challenge. Am J Respir Cell Mol Biol 43: 452-457, 2010.

139. Patel HJ, Belvisi MG, Donnelly LE, Yacoub MH, Chung KF and Mitchell JA. Constitutive expressions of type I NOS in human airway smooth muscle cells: evidence for an antiproliferative role. FASEB J 13: 1810-1816, 1999.

140. Pena F and Ordaz B. Non-selective cation channel blockers: potential use in nervous system basic research and therapeutics. Mini Rev Med Chem 8: 812-819, 2008.

141. Post MJ, Te Biesebeek JD, Doods HN, Wemer J, Van Rooji HH and Porsius AJ. Functional characterization of the muscarinic receptor in rat lungs. Eur J Pharmacol 202: 67-72, 1991.

142. Prakash Y, Thompson MA, Meuchel L, Pabelick CM, Mantilla CB, Zaidi S and Martin RJ. Neurotrophins in lung health and disease. Expert Rev Respir Med 4: 395-411, 2010.

143. Renz H. Neurotrophins in bronchial asthma. Respir Res 2: 265-268, 2001.

144. Ressmeyer AR. Mechanisms of bronchoconstriction in the early allergic response (Dissertation). University of Lübeck, 2006.

145. Ressmeyer AR, Larsson AK, Vollmer E, Dahlen SE, Uhlig S and Martin C. Characterization of guinea pig precision-cut lung slices: comparison with human tissues. Eur Respir J 28: 603$611,2006$.

146. Richardson J and Beland J. Nonadrenergic inhibitory nervous system in human airways. J Appl Physiol 41: 764-771, 1976.

147. Rodger IW. Asthma. Airway smooth muscle. Br Med Bull 48: 97-107, 1992.

148. Rotundo RL. Expression and localization of acetylcholinesterase at the neuromuscular junction. Journal of Neurocytology 32: 743-766, 2003.

149. Russell WMS and Burch RL. The principles of human experimental technique. Universities Federation for Animal Welfare (UFAW), 1959. 
150. Saroea HG, Inman MD and O'Byrne PM. U46619-induced bronchoconstriction in asthmatic subjects is mediated by acetylcholine release. Am J Respir Crit Care Med 151: 321 324, 1995.

151. Schlepütz M, Dassow C, Kramer BW, König P, Uhlig S and Martin C. Comparison of airway responses in sheep of different age in precision-cut lung slices (PCLS). Am J Respir Crit Care Med 181: A5031, 2010.

152. Schlepütz $\mathbf{M}$, Uhlig S and Martin C. Electric field stimulation of precision-cut lung slices. J Appl Physiol 110: 545-554, 2011.

153. Schneider T, van VD, Moqbel R and Issekutz AC. Kinetics and quantitation of eosinophil and neutrophil recruitment to allergic lung inflammation in a brown Norway rat model. Am J Respir Cell Mol Biol 17: 702-712, 1997.

154. Schraufnagel DE. Breathing in America: diseases, progress, and hope. American Thoracic Society, 2010.

155. Seehase S, Schlepütz M, Switalla S, Mätz-Rensing K, Kaup FJ, Zöller M, Schlumbohm C, Fuchs E, Lauenstein HD, Winkler C, Kuehl AR, Uhlig S, Braun A, Sewald K and Martin C. Bronchoconstriction in non-human primates: a species comparison. J Appl Physiol, in press, 2011.

156. Sheller JR and Brigham KL. Bronchomotor responses of isolated sheep airways to electrical field stimulation. J Appl Physiol 53: 1088-1093, 1982.

157. Singh P, Daniels M, Winsett DW, Richards J, Doerfler D, Hatch G, Adler KB and Gilmour MI. Phenotypic comparison of allergic airway responses to house dust mite in three rat strains. Am J Physiol Lung Cell Mol Physiol 284: L588-L598, 2003.

158. Spina D, Matera GM, Riccio MM and Page CP. A comparison of sensory nerve function in human, guinea-pig, rabbit and marmoset airways. Life Sci 63: 1629-1642, 1998.

159. Stefaniak MS, Krumdieck CL, Spall RD, Gandolfi AJ and Brendel K. Biochemical and histological characterization of agar-filled precision-cut rat lung slices in dynamic organ culture as an in vitro tool. In Vitro Toxicology 5: 7-19, 1992.

160. Sturton G, Persson C and Barnes PJ. Small airways: an important but neglected target in the treatment of obstructive airway diseases. Trends Pharmacol Sci 29: 340-345, 2008. 
161. Sundstrom E, Lastbom L, Ryrfeldt A and Dahlen SE. Interactions among three classes of mediators explain antigen-induced bronchoconstriction in the isolated perfused and ventilated guinea pig lung.J Pharmacol Exp Ther 307: 408-418, 2003.

162. Sutherland ER and Martin RJ. Targeting the distal lung in asthma: do inhaled corticosteroids treat all areas of inflammation? Treat Respir Med 4: 223-229, 2005.

163. Switalla S, Lauenstein L, Prenzler F, Knothe S, Forster C, Fieguth HG, Pfennig O, Schaumann F, Martin C, Guzman CA, Ebensen T, Muller M, Hohlfeld JM, Krug N, Braun A and Sewald K. Natural innate cytokine response to immunomodulators and adjuvants in human precision-cut lung slices. Toxicol Appl Pharmacol 246: 107-115, 2010.

164. Szarek JL and Spurlock B. Antagonism of cholinergic nerve-mediated contractions by the sensory nerve inhibitory system in rat bronchi. J Appl Physiol 81: 260-265, 1996.

165. Szarek JL, Stewart NL, Spurlock B and Schneider C. Sensory nerve- and neuropeptidemediated relaxation responses in airways of Sprague-Dawley rats. J Appl Physiol 78: 16791687, 1995.

166. Szelenyi I. Animal models of bronchial asthma. Inflamm Res 49: 639-654, 2000.

167. Takata S, Aizawa H, Shigyo M, Matsumoto K, Inoue H, Koto H and Hara N. Thromboxane A2 mimetic ( $U$-46619) induces hyperresponsiveness of smooth muscle in the canine bronchiole, but not in the trachea. Prostaglandins Leukot Essent Fatty Acids 54: 129-134, 1996.

168. Ten Berge RE, Roffel AF and Zaagsma J. The interaction of selective and non-selective antagonists with pre- and postjunctional muscarinic receptor subtypes in the guinea pig trachea. Eur J Pharmacol 233: 279-284, 1993.

169. Tracey KJ. The inflammatory reflex. Nature 420: 853-859, 2002.

170. Uhlig S and Martin C. Bronchial Asthma. In: Encyclopedia of Molecular Pharmacology, edited by Offermanns S and Rosenthal W. Springer, 2008.

171. Ulloa L. The vagus nerve and the nicotinic anti-inflammatory pathway. Nat Rev Drug Discov 4: 673-684, 2005. 
172. Undem BJ and Carr MJ. The role of nerves in asthma. Curr Allergy Asthma Rep 2: 159-165, 2002.

173. Undem BJ, Hunter DD, Liu $M$, Haak-Frendscho $M$, Oakragly A and Fischer A. Allergeninduced sensory neuroplasticity in airways. Int Arch Allergy Immunol 118: 150-153, 1999.

174. Undem BJ and Kollarik $\mathbf{M}$. The role of vagal afferent nerves in chronic obstructive pulmonary disease. Proc Am Thorac Soc 2: 355-360, 2005.

175. Undem BJ, Myers AC, Barthlow H and Weinreich D. Vagal innervation of guinea pig bronchial smooth muscle. J Appl Physiol 69: 1336-1346, 1990.

176. Underwood DC, Muccitelli RM, Luttmann MA, Hay DW, Torphy TJ and Wasserman MA. Differential antagonism of airway contractile responses to prostaglandin (PG)D2 and 9 alpha, 11 beta-PGF2 by atropine, SK\&F 88046 and SQ 29,548 in the guinea pig. J Pharmacol Exp Ther 268: 304-310, 1994.

177. van den Berge M, Ten Hacken NH, Cohen J, Douma WR and Postma DS. Small airway disease in asthma and COPD: clinical implications. Chest 139: 412-423, 2011.

178. van der Velden $\mathbf{V}$ and Hulsmann AR. Autonomic innervation of human airways: structure, function, and pathophysiology in asthma. Neuroimmunomodulation 6: 145-159, 1999.

179. Verbout NG, Jacoby DB, Gleich GJ and Fryer AD. Atropine-enhanced, antigen challengeinduced airway hyperreactivity in guinea pigs is mediated by eosinophils and nerve growth factor. Am J Physiol Lung Cell Mol Physiol 297: L228-L237, 2009.

180. Verhein KC, Hazari MS, Moulton BC, Jacoby IW, Jacoby DB and Fryer AD. Three days after a single exposure to ozone, the mechanism of airway hyperreactivity is dependent on substance P and nerve growth factor. Am J Physiol Lung Cell Mol Physiol 300: L176-L184, 2011.

181. Vietmeier J, Niedorf F, Baumer W, Martin C, Deegen E, Ohnesorge B and Kietzmann M. Reactivity of equine airways--a study on precision-cut lung slices. Vet Res Commun 31: 611619, 2007.

182. Waelbroeck M, Robberecht P, Coy DH, Camus JC, de NP and Christophe J. Interaction of growth hormone-releasing factor (GRF) and 14 GRF analogs with vasoactive intestinal peptide (VIP) receptors of rat pancreas. Discovery of (N-Ac-Tyr1,D-Phe2)-GRF(1-29)-NH2 as a VIP antagonist. Endocrinology 116: 2643-2649, 1985. 
183. Wang Z, Robinson NE and Yu M. ACh release from horse airway cholinergic nerves: effects of stimulation intensity and muscle preload. Am J Physiol 264: L269-L275, 1993.

184. Watson N, Magnussen $\mathbf{H}$ and Rabe KF. The relevance of resting tension to responsiveness and inherent tone of human bronchial smooth muscle. Br J Pharmacol 123: 694-700, 1998.

185. Wegmann M, Fehrenbach H, Fehrenbach A, Held T, Schramm C, Garn H and Renz H. Involvement of distal airways in a chronic model of experimental asthma. Clin Exp Allergy 35: 1263-1271, 2005.

186. Wettschureck $\mathbf{N}$ and Offermanns $\mathbf{S}$. Rho/Rho-kinase mediated signaling in physiology and pathophysiology. J Mol Med 80: 629-638, 2002.

187. Wohlsen A, Hirrle A, Tenor H, Marx D and Beume R. Effect of cyclic AMP-elevating agents on airway ciliary beat frequency in central and lateral airways in rat precision-cut lung slices. Eur J Pharmacol 635: 177-183, 2010.

188. Wohlsen A, Martin C, Vollmer E, Branscheid D, Magnussen H, Becker WM, Lepp U and Uhlig $\mathbf{S}$. The early allergic response in small airways of human precision-cut lung slices. Eur Respir J 21: 1024-1032, 2003.

189. Wohlsen A, Uhlig S and Martin C. Immediate allergic response in small airways. Am J Respir Crit Care Med 163: 1462-1469, 2001.

190. Wu ZX and Dey RD. Nerve growth factor-enhanced airway responsiveness involves substance P in ferret intrinsic airway neurons. Am J Physiol Lung Cell Mol Physiol 291: L111-L118, 2006.

191. Yeh HC, Schum GM and Duggan MT. Anatomic models of the tracheobronchial and pulmonary regions of the rat. Anat Rec 195: 483-492, 1979. 



\section{Lebenslauf}

\section{Persönliche Daten}

$\begin{array}{ll}\text { Name: } & \text { Schlepütz } \\ \text { Vorname: } & \text { Marco } \\ \text { Geburtstag: } & \text { 20. Januar } 1982 \\ \text { Geburtsort: } & \text { Simmerath } \\ \text { Staatsangehörigkeit: } & \text { Deutsch }\end{array}$

\section{Qualifikationen}

2001

$2002-2005$

$2005-2007$

Seit 2007
Abitur am Burgau Gymnasium, Stadt Düren

Bachelorstudium Biotechnologie / molekulare Biotechnologie an der RWTH Aachen University

Masterstudium Biotechnologie / molekulare Biotechnologie an der RWTH Aachen University

Promotion am Institut für Pharmakologie und Toxikologie des Universitätsklinikums Aachen der RWTH Aachen University 